\title{
Magnetic resonance spectroscopy to unravel metabolic alterations in hepatic steatosis in humans
}

Citation for published version (APA):

Veeraiah, P. (2021). Magnetic resonance spectroscopy to unravel metabolic alterations in hepatic steatosis in humans. [Doctoral Thesis, Maastricht University]. Ridderprint. https://doi.org/10.26481/dis.20210701pv

Document status and date:

Published: 01/01/2021

DOI:

10.26481/dis.20210701pv

Document Version:

Publisher's PDF, also known as Version of record

\section{Please check the document version of this publication:}

- A submitted manuscript is the version of the article upon submission and before peer-review. There can be important differences between the submitted version and the official published version of record.

People interested in the research are advised to contact the author for the final version of the publication, or visit the DOI to the publisher's website.

- The final author version and the galley proof are versions of the publication after peer review.

- The final published version features the final layout of the paper including the volume, issue and page numbers.

Link to publication

\footnotetext{
General rights rights.

- You may freely distribute the URL identifying the publication in the public portal. please follow below link for the End User Agreement:

www.umlib.nl/taverne-license

Take down policy

If you believe that this document breaches copyright please contact us at:

repository@maastrichtuniversity.nl

providing details and we will investigate your claim.
}

Copyright and moral rights for the publications made accessible in the public portal are retained by the authors and/or other copyright owners and it is a condition of accessing publications that users recognise and abide by the legal requirements associated with these

- Users may download and print one copy of any publication from the public portal for the purpose of private study or research.

- You may not further distribute the material or use it for any profit-making activity or commercial gain

If the publication is distributed under the terms of Article $25 \mathrm{fa}$ of the Dutch Copyright Act, indicated by the "Taverne" license above, 


\section{Magnetic resonance spectroscopy to unravel metabolic alterations in hepatic steatosis in humans}




\section{Health Holland}

Portal to the Dutch Life

Sciences \& Health sector

The research presented in this thesis was performed within NUTRIM School of Nutrition and Translational Research in Metabolism. The research described in this thesis was conducted within the Health Holland framework and was financially supported by Unilever R\&D Wageningen and Health Holland.

Cover design: Sandra Tukker (ridderprint) and Sugapriya Krishnan

Layout: Pandichelvam Veeraiah

Printed by: Ridderprint - ridderprint.nl

ISBN:978-94-6416-610-1

Electronic ISBN: 978-94-6416-607-1

Copyright: Pandichelvam Veeraiah 2021

No parts of this thesis may be reproduced, stored in a retrieval system, or distributed in any form or by any means without the permission of author, or, when appropriate, from the publishers of the manuscript. 


\title{
Magnetic resonance spectroscopy to unravel metabolic alterations in hepatic steatosis in humans
}

\author{
DISSERTATION \\ to obtain the degree of Doctor at the Maastricht University, \\ on the authority of the Rector Magnificus, \\ Prof.dr. Rianne M. Letschert \\ in accordance with the decision of the Board of Deans, \\ to be defended in public \\ on Thursday $1^{\text {st }}$ of July 2021 at 12:00 hours.
}

by

Pandichelvam Veeraiah 


\section{Supervisor(s)}

Dr. Vera B. Schrauwen-Hinderling

Prof. dr. Joachim E. Wildberger

\section{Co-supervisor}

Dr. ir. Lucas Lindeboom (Imec - High Tech Campus Eindhoven)

\section{Assessment committee}

Prof. dr. Ronald P. Mensink (Chair)

Prof. dr. med. Michael Roden (German Diabetes Center, Germany)

Dr. ir. Tom Scheenen (Radboud University Medical Center, Nijmegen)

Prof. dr. Daisy M.A.E Jonkers

Dr. Jacobus F.A. Jansen 


\section{TABLE OF CONTENTS}

$\begin{array}{lll}\text { Chapter 1. General introduction } & 7\end{array}$

Chapter 2. Hepatic water and lipid $\mathrm{T}_{2}$ relaxation times are associated with level of steatosis - implications for absolute fat quantification

Chapter 3. Hepatic saturated fatty acid fraction is associated with de novo lipogenesis and hepatic insulin resistance

Chapter 4. Application of a Bllinear Rotation Decoupling (BIRD) 75 filter in combination with $\mathrm{J}$-difference editing for indirect ${ }^{13} \mathrm{C}$ measurements in the human liver

Chapter 5. Effects of fructose restriction on liver steatosis 91 (FRUITLESS); a double-blind randomized controlled trial

Chapter 6. High intrahepatic lipid content is associated with low choline status in obese volunteers - a ${ }^{1} \mathrm{H}-\mathrm{MRS}$ study

Chapter 7. General discussion

$\begin{array}{lll}\text { Appendices Summary } & 151\end{array}$

Impact of the thesis 157

Acknowledgements 163

$\begin{array}{ll}\text { Scientific output } & 169\end{array}$

$\begin{array}{ll}\text { About the author } & 175\end{array}$ 



\section{CHAPTER 1}

General introduction 


\section{INTRODUCTION}

\section{Scientific relevance and challenges}

Obesity-related metabolic disorders pose a major health burden and are leading to high mortality and morbidity worldwide. According to the World Health Organization (WHO), more than one billion people are overweight $\left(25 \mathrm{~kg} / \mathrm{m}^{2} \leq \mathrm{BMI}\right)$ globally (1) and in Netherlands, more than half of the population is overweight. Moreover, the WHO has estimated that 3.5 million people worldwide die annually due to obesity-related problems including diabetes type II and cardiovascular diseases (CVD) (2,3). In obese/overweight conditions, excessive fat is not only stored in adipose tissue but also accumulates in important organs including the liver, heart, and muscle. Such fat deposits are often referred to as ectopic fat. Growing evidence indicates that ectopic fat accumulation is one of the major determinants for obesity-related metabolic risks $(4,5)$. Quite strikingly, 20 to $30 \%$ of the general population in Western countries have excessive storage of fat in the liver, in the absence of high alcohol intake, the abnormal intrahepatic lipid (IHL) storage, primarily as triglycerides (TG) within hepatocytes is referred to as non-alcoholic fatty liver (NAFL) or hepatic steatosis (2). As the obesity epidemic grows, the prevalence of NAFL is increasing rapidly worldwide, even in developing countries with an estimated prevalence rate of 50-70\% in obese/overweight populations (1). NAFL is the initial and reversible stage of a disease spectrum, termed non-alcoholic fatty liver disease (NAFLD), which can progress to non-alcoholic steatohepatitis (NASH), fibrosis and the irreversible stages of chronic liver diseases such as cirrhosis (6). In fact, NAFLD is the second most common risk factor for liver transplantation (7). Moreover, individuals with NAFL have been associated with impairments in insulin sensitivity and high risk to develop diabetes type II (8). Despite the increasing predominance and known adverse effects of NAFL, the mechanistic details in the development and progression of NAFL are still poorly understood, relying mainly on data from animal studies. A principal reason for the incomplete understanding of the pathophysiology of NAFL in humans is the lack of appropriate noninvasive methodologies to study hepatic lipid metabolism.

\section{Metabolic pathways in the liver}

In normal physiology, the liver plays a central role in maintaining metabolic homeostasis by regulating constant energy supply to the body in both fed and fasted states through metabolism of nutrients (carbohydrates and lipids). Fatty acids (FAs) can reach the liver from three different common metabolic pathways as shown in the Figure 1. In the fed state, fatty acids are delivered into the liver as chylomicrons from the intestine that are derived from consumption 
of dietary fat. Alternatively, fatty acids can enter into the liver from adipose tissue through the process of lipolysis (in post-absorptive state). In addition to these exogenous fatty acid influxes into the liver, fatty acids can also be synthesized in the liver itself through the process of de novo lipogenesis (DNL) from non-lipid precursors (e.g., glucose, fructose, amino acids and glycerol).

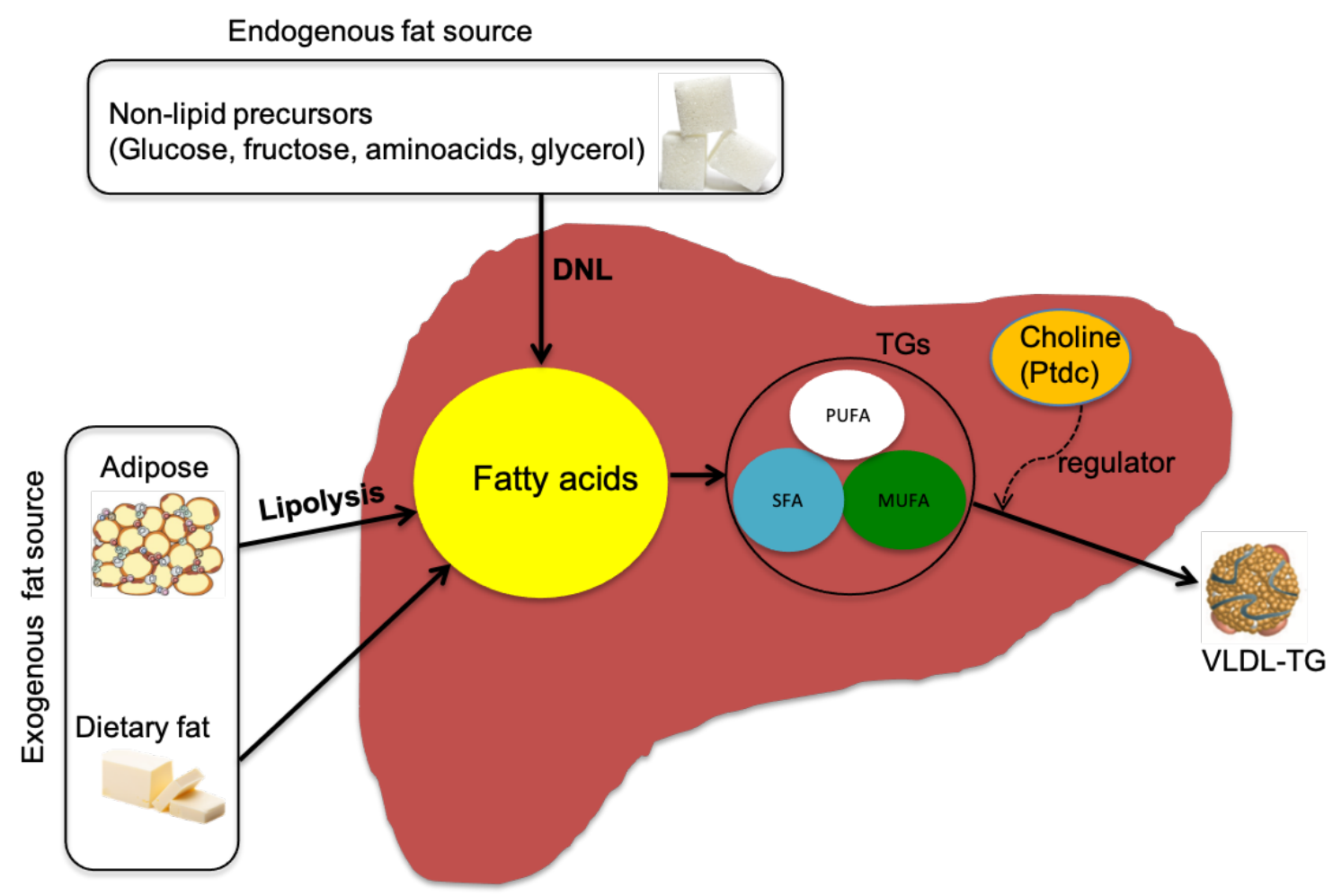

Figure 1: Schematic representation of different metabolic pathways involved in hepatic fat accumulation. TGs-Triglycerides; DNL-De novo lipogenesis; PtdC-Phosphatidylcholine; VLDL-TGVery low-density lipoprotein triglyceride; SFA-Saturated fatty acids; MUFA-Monounsaturated fatty acids; PUFA-Polyunsaturated fatty acids.

Finally, the entered/synthesized fatty acids can have two fates, the first is storage in the liver, primarily as triglycerides (TGs), which can be differentiated into saturated, mono and polyunsaturated fatty acids (SFA, MUFA, PUFA). Secondly, the FAs can be removed from the liver through fat disposal pathways such as mitochondrial fatty acid oxidation or secretion of very-low density lipoproteins (VLDL) (9). When there is an imbalance between the influx and efflux of fat in the liver, with influx outweighing efflux, net hepatic fat storage occurs and leads to liver fattening (10). Interestingly, the endogenous production of FAs in the liver through DNL results mainly in saturated fatty acids (SFA) $(11,12)$. Therefore, determining SFA fraction in the liver may provide an indirect measure of DNL in humans. Although experimental research on animals and in vitro studies have provided us valuable mechanistic insights by identifying the metabolic pathways involved in the etiology of NAFL, the 
translation to humans is challenging and their relevance for humans often remains unclear. This is mainly due to the fact that tissue biopsies are generally needed to obtain metabolic information, but such invasive techniques are undesirable and inappropriate for longitudinal studies in humans. Therefore, reliable and quantitative non-invasive methodologies are warranted to study metabolic pathways leading to NAFL in humans, which could be instrumental in understanding the pathophysiology and evaluating the mechanism of action and efficacy of potential treatments.

\section{Role of magnetic resonance spectroscopy (MRS) in investigating hepatic lipid metabolism}

So far, several techniques have been developed for the detection of hepatic steatosis and quantification of intrahepatic lipid (IHL) content. The conventional histology method is a visual, semi-quantitative approach in which steatosis is diagnosed when $>5 \%$ of hepatocytes are filled with macro vesicular steatosis (13). Alternatively, a biochemical method can be used to assess hepatic steatosis after lipid extraction. Using biochemical analysis, the normal healthy IHL content is defined as below 5.5\% of TG of wet liver tissue weight (14). All these tissuebased measures of IHL content rely on liver biopsy, which is invasive, subjective to sampling error and is therefore not suitable for risk stratification and effective management of widespread NAFL $(15,16)$. Non-invasive techniques such as computed tomography (CT) and ultra-sonography (US) can be used to diagnose NAFL, but exact absolute IHL quantification is difficult (17-19). Furthermore, the use of ionization radiation in CT is a drawback for the use in patients. In contrast, non-invasive magnetic resonance (MR) based methods such as MR spectroscopy (MRS) and MR imaging (MRI) are rapidly evolving as efficient tools for assessing hepatic steatosis (20-23). MRS provides biochemical information on cellular components and is considered a powerful tool to study metabolism in vivo in a non-invasive manner. Nowadays, proton magnetic resonance spectroscopy ( $\left.{ }^{1} \mathrm{H}-\mathrm{MRS}\right)$ is considered the gold standard method for non-invasive measurements to determine IHL content. ${ }^{1} \mathrm{H}-\mathrm{MRS}$ has been widely used in both experimental and clinical settings as an alternative tool for liver biopsy for the detection of hepatic steatosis $(20-22,24)$. However, traditional ${ }^{1} \mathrm{H}-\mathrm{MRS}$ is not used to its full potential. Investing time in optimizing MR protocols, developing new MR sequences, and improving post-processing approaches could provide access to more valuable metabolic information. Therefore, the research work in this $\mathrm{PhD}$ thesis is mainly focused on the development and application of new MRS protocols to study hepatic lipid metabolism. This would eventually allow the translation of pre-clinical knowledge to humans towards understanding the metabolic pathways leading to hepatic steatosis. 


\section{OUTLINE OF THE THESIS}

\section{Intrahepatic lipid content and NAFL}

As mentioned before, ${ }^{1} \mathrm{H}-\mathrm{MRS}$ is widely used to measure IHL content. ${ }^{1} \mathrm{H}$-MRS in the liver enables sampling of a large volume fraction (typically $\sim 10 \mathrm{~mL}$ ) of liver tissue referred to as "voxel" and the net MR signal is derived from protons in both triglycerides and water within the voxel. Thus, ${ }^{1} \mathrm{H}-\mathrm{MRS}$ determines the volumetric fat fraction rather than the percentage of hepatocytes with macroscopic lipid droplets as visualized by histology. Therefore, it is important to provide the final outcome of MRS to standardized clinical measures of IHL (25) such as weight/weight percentages comparable to biochemical analysis of biopsies as described above. In order to convert MRS signal intensities of water and fat to weight/weight $\%$, several factors need to be considered. Unfortunately, there are many physical, biological, and technical factors which can affect the actual MR signal intensities of both fat and water, which ultimately can lead to substantial bias in the estimation of absolute IHL content. Such confounding factors can for example be $\mathrm{T}_{1}$ bias, $\mathrm{T}_{2}$ relaxation, spectral complexity of the fat spectrum, J-coupling and eddy currents (26). All these confounding factors should be addressed and need to be corrected for the accurate estimation of IHL content. Moreover, there is also variation in the usage of different fat quantification methods/formulas $(8,27-29)$ and difference in the way of expressing IHL\% either as a simple ratio $(28,30)$ or in absolute terms $(20,29)$. This leads to confusion for the general public as well as the scientific community to understand what exactly is meant by IHL\% and how it is related to the known cut-off value that defines NAFL. In chapter 2, we review/addressed all the steps that are taken to convert the MRS-based signal to IHL in absolute terms $(\mathrm{w} / \mathrm{w})$. In addition, we evaluate the bias that occurs in the quantification of IHL \% when using a slightly incorrect factor for $\mathrm{T}_{2}$ relaxation and show how such bias can be minimized.

\section{Intrahepatic lipid composition and NAFL}

Apart from determining the total IHL content, it is also important to characterize the fatty acid composition and differentiate saturated, mono and poly-unsaturated fatty acids (SFA, MUFA, PUFA) in the liver. Data from in vitro and animal studies have shown that SFA specifically may be implicated in the development of insulin resistance while unsaturated fatty acids are not linked to the typical adverse effects of lipids such as disturbance of insulin sensitivity $(31,32)$. Next to having potentially negative health effects, there is an additional interesting aspect to quantifying SFA in the liver: the degree of hepatic saturation of FAs is likely to be modulated by the rate of de novo lipogenesis since SFAs are the main products of DNL. 


\section{$\underline{\text { CHAPTER } 1}$}

Therefore, instead of quantifying only the total IHL content, distinguishing the type of fat stored in the liver provides new mechanistic insights.

Determining the hepatic lipid composition is not straightforward with conventional ${ }^{1} \mathrm{H}-\mathrm{MRS}$, because at clinical field strength (3T) it is challenging to characterize the type of lipid pool due to poor spectral resolution. The typical ${ }^{1} \mathrm{H}-\mathrm{MR}$ derived spectrum of the liver acquired at $3 \mathrm{~T}$ resolves only four single lipid resonances that are of analytical interest: the methyl (peak a), methylene (peak b), diallylic (peak e) and olefinic/methine (peak f) peak, as shown in Figure $2 \mathrm{~B}$.

A) Chemical structure of triglyceride

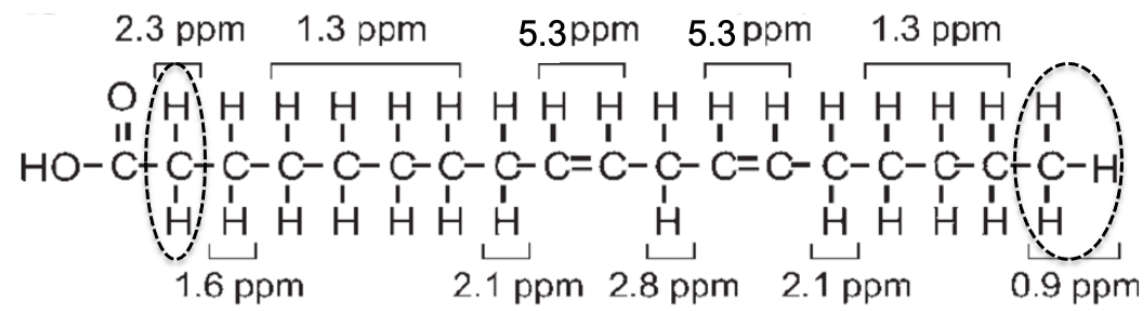

B) Typical ${ }^{1} \mathrm{H}$ - MR liver spectrum

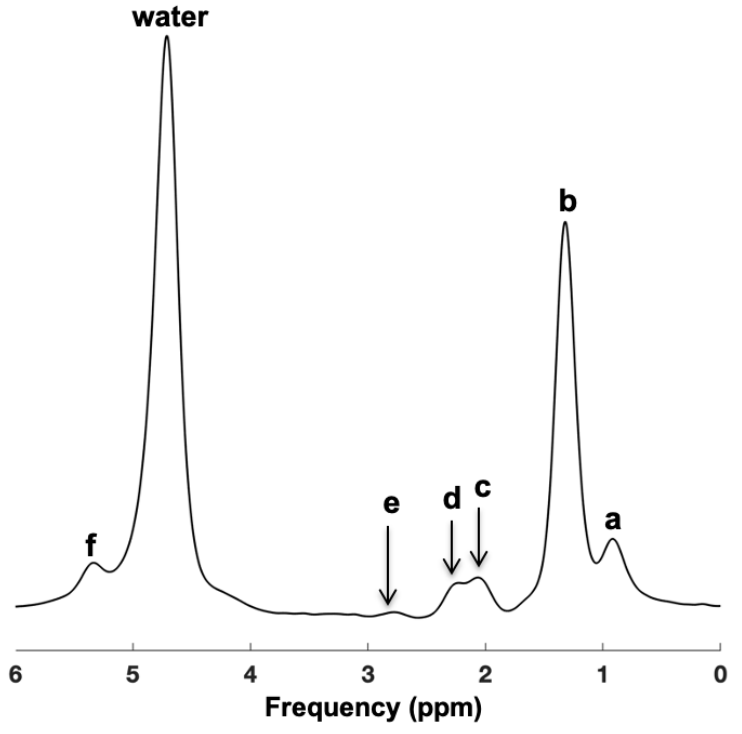

C) Lipid peaks and position

\begin{tabular}{|l|c|}
\hline \multicolumn{1}{|c|}{ Lipid protons } & $\begin{array}{c}\text { Position } \\
\text { (ppm) }\end{array}$ \\
\hline a. Methyl $\left(-\mathrm{CH}_{3}\right)$ & 0.9 \\
\hline b. Methylene $\left(-\mathrm{CH}_{2}\right)_{\mathrm{n}}$ & 1.3 \\
\hline c. Allylic $\left(-\mathrm{CH}_{2}-\mathrm{CH}=\mathrm{CH}-\mathrm{CH}_{2}-\right)$ & 2.1 \\
\hline d. Alpha-methylene $\left(-\mathrm{CO}-\mathrm{CH}_{2}-\mathrm{CH}_{2}-\right)$ & 2.3 \\
\hline e. Diallylic $(-\mathrm{CH}=\mathrm{CH}-\mathrm{CH} 2-\mathrm{CH}=\mathrm{CH}-)$ & 2.8 \\
\hline f. Methine $(-\mathrm{CH}=\mathrm{CH}-\mathrm{CH} 2-\mathrm{CH}=\mathrm{CH}-)$ & 5.3 \\
\hline
\end{tabular}

Figure 2: Chemical structure of a triglyceride molecule and a typical ${ }^{1} \mathrm{H}-\mathrm{MR}$ hepatic spectrum acquired at 3T. A) Schematic representation of a triglyceride molecule in which middle fatty acid is shown in detail with all lipid protons. B) Typical ${ }^{1} \mathrm{H}-\mathrm{MR}$ derived liver spectrum showing all the corresponding lipid peaks and positions. C) Table shows the name of all lipid peaks and its position. Black dotted circle in Figure 2A represents the two different lipid protons (corresponding peak a \& d) which occur in every fatty acid molecule- one of the main prior knowledge used to determine lipid composition. 
The olefinic resonance arises from protons attached to the double-bonded carbons, and their abundance correlates with the average double bond content of all fatty acids in which PUFA contributes twice as much olefinic protons as compared to MUFA. The diallylic resonance arises from the methylene protons between two double-bonded carbons in a fatty acid molecule, which represents PUFA content only. The allylic resonance arises from the methylene protons next to the double-bonded carbons (but not in-between double bonds), and MUFA and PUFA have the same number of allylic protons. In principle, the distinct detection of the allylic and diallylic peak allows estimating the MUFA and PUFA content separately, but at $3 \mathrm{~T}$, the allylic peak is contaminated with the alpha-carbonyl methylene resonance as shown in the spectrum of Figure 2B (peak $\mathrm{c}$ and d), which hampers the accurate measurement of hepatic lipid composition. Therefore, an optimized technique to tackle this problem is required. To achieve this, we optimized the MR acquisition and post-processing protocols for regular ${ }^{1} \mathrm{H}-\mathrm{MRS}$ of the liver. We developed a new post-processing tool to determine hepatic lipid composition using extensive prior knowledge to overcome the limited resolution of the allylic peak and the alpha-carbonyl methylene peak. Furthermore, the developed ${ }^{1} \mathrm{H}-\mathrm{MRS}$ protocol was successfully applied in a clinical study to measure hepatic lipid composition in volunteers with a wide range of liver fat content to explore the relationship between hepatic lipid composition, insulin sensitivity and DNL. This is addressed in chapter 3.

\section{Indirect ${ }^{13} \mathrm{C}$ MRS for ${ }^{13} \mathrm{C}$ tracking applications in the liver}

Although ${ }^{1} \mathrm{H}-\mathrm{MRS}$ is a very valuable tool to quantify IHL content and lipid composition, this method cannot distinguish between exogenous and endogenous lipids and therefore can only determine net changes, being unable to determine metabolic fluxes (29). In contrast, ${ }^{13} \mathrm{C} \mathrm{MR}$ methodology together with specific ${ }^{13} \mathrm{C}$ labeled substrates enables us to track the incorporation into the low natural abundance of ${ }^{13} \mathrm{C}(1.1 \%)$ in the hepatic lipid pool, which helps to obtain novel insights into the pathways involved in NAFL. However, because of the low sensitivity of direct ${ }^{13} \mathrm{C} \mathrm{MR}$, more advanced indirect ${ }^{13} \mathrm{C}$ spectral editing $\mathrm{MR}$ methods, that increase sensitivity and allow for better localization of the signal, have been developed to study ${ }^{13} \mathrm{C}$ enrichment. In such techniques, the main goal is to selectively detect the small number of protons bound to ${ }^{13} \mathrm{C}$ nuclei $(1.1 \%)$ by suppressing the large number of protons $(98.9 \%)$ attached to ${ }^{12} \mathrm{C}$ nuclei, taking advantage of increased sensitivity of ${ }^{1} \mathrm{H}$ as compared to ${ }^{13} \mathrm{C}$. Recently, our research group introduced a quantum coherence-based method (ge-HSQC) for indirect ${ }^{13} \mathrm{C}$-MRS (33) in the liver and demonstrated the in vivo feasibility to track ${ }^{13} \mathrm{C}$-labeled lipids into the hepatic lipid pool in the liver. This approach is more robust for the liver than the 
previously reported J-difference editing (JDE) techniques which are very sensitive to respiratory motion and challenging for direct application in the human liver. However, the successful application of the conventional JDE technique in animals has shown in proof of principle experiments that the conversion of ${ }^{13} \mathrm{C}$-labeled fructose to fatty acids can be followed in vivo and rate of DNL can be directly measured in rodents (34). While for the application in humans the ge-HSQC is more robust than conventional JDE techniques, a drawback of the use of the ge-HSQC (33) methodology, is the inherent signal loss of 50\% when compared to conventional JDE techniques. To further extend this work and to further improve sensitivity of the method, we developed a new approach for ${ }^{13} \mathrm{C}$ tracking applications in the human liver by improving the robustness of a regular J-difference editing (JDE) technique using BIlinear Rotation Decoupling (BIRD) filter. We demonstrated the in vivo feasibility of our proposed approach and successfully quantified even the low natural abundance of ${ }^{1} \mathrm{H}-\left[{ }^{13} \mathrm{C}\right]$ hepatic lipid signals in healthy volunteers, which is addressed in chapter 4 . Therefore, a reliable indirect ${ }^{13} \mathrm{C}$ spectral editing MR method, with improved sensitivity, was developed for ${ }^{13} \mathrm{C}$ tracking applications in the human liver, towards identifying metabolic pathways involved in NAFL.

It has been demonstrated that not only exogenous dietary fatty acids can induce NAFL, but also consumption of simple sugars can lead to NAFL through DNL. Nowadays, the increased consumption of glucose and fructose in the diet of Western style has drawn the attention towards the role of DNL in hepatic steatosis and especially fructose has gained increased attention in understanding the pathophysiology of NAFL. It is known that fructose can serve as a substrate for de novo lipogenesis. There are indications from animal studies that fructose enhances hepatic DNL $(35,36)$, but very limited data is available on humans to show the detrimental effect of fructose in liver health. The effect of lowering dietary fructose was investigated in chapter 5 by performing a randomized control design study in obese/overweight populations to investigate the effects of 6-week fructose restrictions on IHL content.

\section{Choline and NAFL}

Next to the pathways that contribute to lipid synthesis, which may be increased in NAFL, it is also possible that fat disposal pathways, that usually lead to a decrease in liver fat, may be hampered. It has been shown from animal studies that choline is necessary for synthesis of phosphatidylcholine (PtdC) (37), which is required for packaging and export of VLDL from the liver (38) as depicted in Figure 1. There are indications from animal studies that choline 
deprivation leads to an increase in IHL content and it has been suggested that disturbances in choline metabolism may accelerate the development of NAFL (39-41). However, so far, experimental human data on hepatic choline status and its relationship with NAFL is scarce. The trimethyl-ammonium (TMA) group of choline can be detected non-invasively in the ${ }^{1} \mathrm{H}$ MR derived spectrum at $3.20 \mathrm{ppm}$. Although the TMA peak is visible in the ${ }^{1} \mathrm{H}-\mathrm{MRS}$ spectrum, the quantification of hepatic choline/TMA content is challenging due to low signal when compared to the large resonances of water and lipids. Therefore, better water suppression techniques and robust post-processing are warranted for the accurate detection and quantification of the choline peak. With the help of a post-processing tool previously developed by us (to determine lipid composition addressed in chapter 3 ), we showed the in vivo feasibility of hepatic choline quantification in chapter 6 and evaluated the relationship of hepatic choline with IHL content and composition in obese/overweight populations.

Finally, in chapter 7, major results, and conclusions of all the previous chapters in this thesis are discussed in a broader perspective. In addition, future directions of metabolic research with the use of in vivo MRS and applications of our developed MRS protocols are discussed. 


\section{REFERENCES}

1. Younossi ZM. Non-alcoholic fatty liver disease - A global public health perspective. J Hepatol. 2019;70(3):531-44.

2. Loomba R, Sanyal AJ. The global NAFLD epidemic. Nat Rev Gastroenterol Hepatol. 2013;10(11):686-90.

3. Younossi Z, Anstee QM, Marietti M, Hardy T, Henry L, Eslam M, et al. Global burden of NAFLD and NASH: trends, predictions, risk factors and prevention. Nat Rev Gastroenterol Hepatol. 2018 Jan;15(1):1120.

4. Rasouli N, Molavi B, Elbein SC, Kern PA. Ectopic fat accumulation and metabolic syndrome. Diabetes Obes Metab. 2007 Jan;9(1):1-10.

5. Morelli M, Gaggini M, Daniele G, Marraccini P, Sicari R, Gastaldelli A. Ectopic fat: the true culprit linking obesity and cardiovascular disease? Thromb Haemost. 2013 Oct;110(4):651-60.

6. Calzadilla Bertot L, Adams LA. The Natural Course of Non-Alcoholic Fatty Liver Disease. Int J Mol Sci. 2016 May 20;17(5).

7. Chedid MF. Nonalcoholic Steatohepatitis: The Second Leading Indication for Liver Transplantation in the USA. Dig Dis Sci. 2017;62(10):2621-2.

8. Brouwers B, Schrauwen-Hinderling VB, Jelenik T, Gemmink A, Havekes B, Bruls Y, et al. Metabolic disturbances of non-alcoholic fatty liver resemble the alterations typical for type 2 diabetes. Clin Sci Lond Engl 1979. 2017 Aug 1;131(15):1905-17.

9. Berlanga A, Guiu-Jurado E, Porras JA, Auguet T. Molecular pathways in non-alcoholic fatty liver disease. Clin Exp Gastroenterol. 2014;7:221-39.

10. Leite NC, Villela-Nogueira CA, Cardoso CRL, Salles GF. Non-alcoholic fatty liver disease and diabetes: From physiopathological interplay to diagnosis and treatment. World J Gastroenterol WJG. 2014 Jul 14;20(26):8377-92.

11. Hellerstein MK, Schwarz JM, Neese RA. Regulation of hepatic de novo lipogenesis in humans. Annu Rev Nutr. 1996;16:523-57.

12. Lambert JE, Ramos-Roman MA, Browning JD, Parks EJ. Increased de novo lipogenesis is a distinct characteristic of individuals with nonalcoholic fatty liver disease. Gastroenterology. 2014 Mar;146(3):72635 .

13. Donhoffer H. Quantitative estimation of lipids in needle biopsy sized specimens of cadaver liver. Acta Med Acad Sci Hung. 1974;31(1-2):47-9.

14. Hoyumpa AM, Greene HL, Dunn GD, Schenker S. Fatty liver: biochemical and clinical considerations. Am J Dig Dis. 1975 Dec;20(12):1142-70.

15. Gunn NT, Shiffman ML. The Use of Liver Biopsy in Nonalcoholic Fatty Liver Disease: When to Biopsy and in Whom. Clin Liver Dis. 2018;22(1):109-19.

16. Ratziu V, Charlotte F, Heurtier A, Gombert S, Giral P, Bruckert E, et al. Sampling variability of liver biopsy in nonalcoholic fatty liver disease. Gastroenterology. 2005 Jun;128(7):1898-906.

17. Park SH, Kim PN, Kim KW, Lee SW, Yoon SE, Park SW, et al. Macrovesicular hepatic steatosis in living liver donors: use of CT for quantitative and qualitative assessment. Radiology. 2006 Apr;239(1):105-12.

18. Debongnie JC, Pauls C, Fievez M, Wibin E. Prospective evaluation of the diagnostic accuracy of liver ultrasonography. Gut. $1981 \mathrm{Feb} ; 22(2): 130-5$. 
19. Singh D, Das CJ, Baruah MP. Imaging of non alcoholic fatty liver disease: A road less travelled. Indian J Endocrinol Metab. 2013 Nov;17(6):990-5.

20. Szczepaniak LS, Nurenberg P, Leonard D, Browning JD, Reingold JS, Grundy S, et al. Magnetic resonance spectroscopy to measure hepatic triglyceride content: prevalence of hepatic steatosis in the general population. Am J Physiol Endocrinol Metab. 2005 Feb;288(2):E462-468.

21. Springer F, Machann J, Claussen CD, Schick F, Schwenzer NF. Liver fat content determined by magnetic resonance imaging and spectroscopy. World J Gastroenterol. 2010 Apr 7;16(13):1560-6.

22. Longo R, Pollesello P, Ricci C, Masutti F, Kvam BJ, Bercich L, et al. Proton MR spectroscopy in quantitative in vivo determination of fat content in human liver steatosis. J Magn Reson Imaging JMRI. 1995 Jun;5(3):281-5.

23. McPherson S, Jonsson JR, Cowin GJ, O'Rourke P, Clouston AD, Volp A, et al. Magnetic resonance imaging and spectroscopy accurately estimate the severity of steatosis provided the stage of fibrosis is considered. J Hepatol. 2009 Aug;51(2):389-97.

24. Thomsen C, Becker U, Winkler K, Christoffersen P, Jensen M, Henriksen O. Quantification of liver fat using magnetic resonance spectroscopy. Magn Reson Imaging. 1994;12(3):487-95.

25. Reeder SB, Hu HH, Sirlin CB. Proton density fat-fraction: a standardized MR-based biomarker of tissue fat concentration. J Magn Reson Imaging JMRI. 2012 Nov;36(5):1011-4.

26. Reeder SB, Cruite I, Hamilton G, Sirlin CB. Quantitative Assessment of Liver Fat with Magnetic Resonance Imaging and Spectroscopy. J Magn Reson Imaging JMRI. 2011 Oct;34(4):729-49.

27. Gambarota G, Tanner M, van der Graaf M, Mulkern RV, Newbould RD. 1H-MRS of hepatic fat using short TR at 3T: SNR optimization and fast T2 relaxometry. Magma N Y N. 2011 Dec;24(6):339-45.

28. Guiu B, Petit J-M, Loffroy R, Ben Salem D, Aho S, Masson D, et al. Quantification of liver fat content: comparison of triple-echo chemical shift gradient-echo imaging and in vivo proton MR spectroscopy. Radiology. 2009 Jan;250(1):95-102.

29. Lindeboom L, Nabuurs CI, Hesselink MKC, Wildberger JE, Schrauwen P, Schrauwen-Hinderling VB. Proton magnetic resonance spectroscopy reveals increased hepatic lipid content after a single high-fat meal with no additional modulation by added protein. Am J Clin Nutr. 2015 Jan;101(1):65-71.

30. Simons N, Debray F-G, Schaper NC, Kooi ME, Feskens EJM, Hollak CEM, et al. Patients With Aldolase B Deficiency Are Characterized by Increased Intrahepatic Triglyceride Content. J Clin Endocrinol Metab. 2019 Nov 1;104(11):5056-64.

31. Han P, Zhang Y-Y, Lu Y, He B, Zhang W, Xia F. Effects of different free fatty acids on insulin resistance in rats. Hepatobiliary Pancreat Dis Int HBPD INT. 2008 Feb;7(1):91-6.

32. Mäkinen S, Nguyen YH, Skrobuk P, Koistinen HA. Palmitate and oleate exert differential effects on insulin signalling and glucose uptake in human skeletal muscle cells. Endocr Connect. 2017 Jun 5;6(5):331-9.

33. Lindeboom L, de Graaf RA, Nabuurs CI, van Ewijk PA, Hesselink MK, Wildberger JE, et al. Quantum coherence spectroscopy to measure dietary fat retention in the liver. JCI Insight. 2016 Aug 18;1(13):e84671.

34. Jonkers R a. M, Geraedts TR, L. J.C. Loon V, Nicolay K, Prompers JJ. Multitissue assessment of in vivo postprandial intracellular lipid partitioning in rats using localized $1 \mathrm{H}-[\mathrm{13C}]$ magnetic resonance spectroscopy. Magn Reson Med. 2012;68(4):997-1006.

35. Koo H-Y, Miyashita M, Cho BHS, Nakamura MT. Replacing dietary glucose with fructose increases ChREBP activity and SREBP-1 protein in rat liver nucleus. Biochem Biophys Res Commun. 2009 Dec 11;390(2):285-9. 
36. Silva JCP, Marques C, Martins FO, Viegas I, Tavares L, Macedo MP, et al. Determining contributions of exogenous glucose and fructose to de novo fatty acid and glycerol synthesis in liver and adipose tissue. Metab Eng. 2019 Dec 1;56:69-76.

37. Sherriff JL, O'Sullivan TA, Properzi C, Oddo J-L, Adams LA. Choline, Its Potential Role in Nonalcoholic Fatty Liver Disease, and the Case for Human and Bacterial Genes. Adv Nutr Bethesda Md. 2016 Jan;7(1):513.

38. Yao ZM, Vance DE. The active synthesis of phosphatidylcholine is required for very low density lipoprotein secretion from rat hepatocytes. J Biol Chem. 1988 Feb 25;263(6):2998-3004.

39. Raubenheimer PJ, Nyirenda MJ, Walker BR. A choline-deficient diet exacerbates fatty liver but attenuates insulin resistance and glucose intolerance in mice fed a high-fat diet. Diabetes. 2006 Jul;55(7):2015-20.

40. Lombardi B, Pani P, Schlunk FF. Choline-deficiency fatty liver: impaired release of hepatic triglycerides. J Lipid Res. 1968 Jul;9(4):437-46.

41. Itagaki H, Shimizu K, Morikawa S, Ogawa K, Ezaki T. Morphological and functional characterization of non-alcoholic fatty liver disease induced by a methionine-choline-deficient diet in C57BL/6 mice. Int J Clin Exp Pathol. 2013;6(12):2683-96. 


\section{CHAPTER 2}

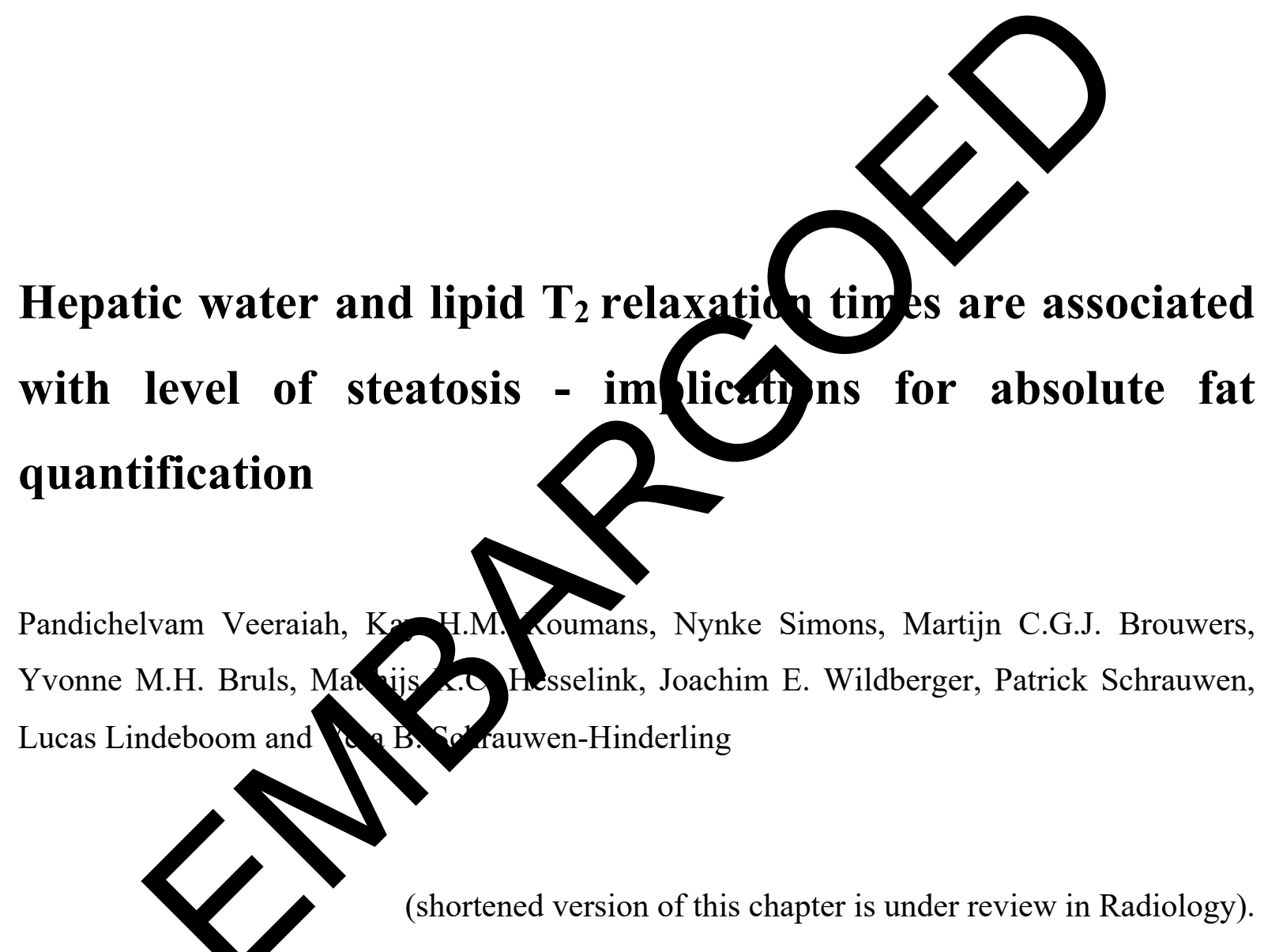


CHAPTER 3

\section{Hepatic saturated fatty acid fraction is associated with de novo lipogenesis and hepatic insulin resistance}

Kay H.M. Roumans ${ }^{\#}$, Lucas Lindeboom, Pandichelvam Veeraiah, Carlijn M.E. Remie, Esther Phielix, Bas Havekes, Yvonne M.H. Bruls, Martijn C.G.J. Brouwers, Marcus Ståhlman, Marjan Alssema, Harry P.F. Peters, Renée de Mutsert, Bart Staels, Marja-Riitta Taskinen, Jan Borén, Patrick Schrauwen and Vera B. Schrauwen-Hinderling

\# These authors contributed equally Nature Communications. 2020 April 20;11(1):1891. 


\section{CHAPTER 3}

\section{ABSTRACT}

Hepatic steatosis is associated with poor cardiometabolic health, with de novo lipogenesis (DNL) contributing to hepatic steatosis and subsequent insulin resistance. Hepatic saturated fatty acids (SFA) may be a marker of DNL and are suggested to be most detrimental in contributing to insulin resistance. Here we show in a cross-sectional study design (ClinicalTrials.gov ID: NCT03211299) that we are able to distinguish the fractions of hepatic SFA, mono- and polyunsaturated fatty acids in healthy and metabolically compromised volunteers using proton magnetic resonance spectroscopy $\left({ }^{1} \mathrm{H}-\mathrm{MRS}\right)$. DNL is positively associated with SFA fraction and is elevated in patients with non-alcoholic fatty liver and diabetes type II. Intriguingly, SFA fraction shows a strong, negative correlation with hepatic insulin sensitivity. Our results show that the hepatic lipid composition, as determined by our ${ }^{1} \mathrm{H}-\mathrm{MRS}$ methodology, is a measure of DNL and suggest that specifically the SFA fraction may hamper hepatic insulin sensitivity. 


\section{INTRODUCTION}

Non-alcoholic fatty liver (NAFL) is the most common cause of chronic liver disease, with estimated prevalence rates of $20-35 \%$ in Western countries (1). In obese people NAFL prevalence rates as high as $50-70 \%$ have been reported (2). NAFL can progress to steatohepatitis, fibrosis, and cirrhosis, which can lead to liver failure, and hepatocellular carcinoma (3). Moreover, ectopic fat accumulation in the liver is associated with impairments in cardiometabolic health $(4,5)$. In this respect, NAFL has been shown to be associated with insulin resistance on hepatic and whole-body level (6-9).

However, not all individuals with NAFL will develop insulin resistance, steatohepatitis or other liver disease, and it is of utmost clinical importance to understand which factors contribute to a pathologic fatty liver. It has been suggested that the pathway by which fat accumulation in the liver occurs may impact the clinical outcome. It has been shown that high rates of de novo lipogenesis (DNL) are associated with metabolic risk (10-12). In addition, animal experiments have suggested that the degree of saturation of the accumulating fatty acids in the liver may impact the metabolic consequences, with more saturated fatty acids (SFA) leading to worsened metabolic outcome (13). Interestingly, the end product of de novo synthesis of fatty acids is mainly SFA and therefore, high rates of DNL may result in a higher proportion of hepatic SFA, possibly explaining why DNL is associated with poor metabolic health. These findings stress the importance for a more detailed characterisation of hepatic lipid composition in humans, to ultimately understand the risk factors for the development of hepatic insulin resistance and disease.

However, in humans such data is very scarce, mainly due to the invasiveness of liver biopsy procedures that are needed. Therefore, only very few studies have determined hepatic fat composition, and these studies have been performed in patients in which liver biopsies were justified due to their risk for liver disease. Generally, these studies showed higher monounsaturated fatty acids (MUFA) fraction at the expense of the poly-unsaturated fatty acids (PUFA) fraction in people with NAFL compared to people without NAFL $(14,15)$. Earlier MRbased studies already investigated some parameters that are linked to degree of unsaturation (16-19), however these do not specifically and robustly quantify hepatic SFA, MUFA and PUFA fraction separately. 


\section{CHAPTER 3}

Here, we develop, validate, and apply a magnetic resonance (MR) post-processing tool that enables to non-invasively quantify the fractions of hepatic SFA, MUFA and PUFA separately, in healthy and metabolically compromised human volunteers. Using this methodology, we test the hypothesis that higher rates of DNL are associated with an increased fraction of SFA in human liver. We also investigate if populations at higher risk to develop metabolic complications are characterized by altered hepatic fatty acid composition, and whether hepatic fatty acid composition is related to hepatic insulin sensitivity. We show that our ${ }^{1} \mathrm{H}-\mathrm{MRS}$ postprocessing methodology can be used to measure hepatic fatty acid composition in humans, that hepatic SFA content is strongly related to rates of DNL and that specifically the hepatic SFA fraction is related to hepatic insulin resistance.

\section{MATERIALS AND METHODS}

\section{Clinical Study Design}

The study was conducted at Maastricht University Medical Center, the Netherlands, between August 2017 and June 2018, and was approved by the Medical Ethical Committee of Maastricht University Medical Centre. Research was performed in accordance with relevant ethical regulations regarding human research participants. The study was registered at clinicaltrials.gov with identifier NCT03211299.

\section{Participants}

All participants recruited for this study provided written informed consent. Twenty-two healthy overweight/obese participants (BMI 27-35 kg/m²) aged 45-70 years with a large range in liver fat content $(0.9-38.4 \%)$ were recruited for this study. Female study participants were postmenopausal. Exclusion criteria were engagement in exercise for more than 2 hours per week, unstable body weight (weight loss or gain more than $3 \mathrm{~kg}$ in 3 months preceding enrollment), alcohol consumption more than 2 units per day, smoking more than 5 cigarettes per day, contra-indication for MRI, use of anti-coagulants, use of other medication known to interfere with the outcome parameters, diabetes or other active disease. Participants participated in an MRS measurement, deuterated water measurement and two-step hyperinsulinemic-euglycemic clamp, including baseline subcutaneous adipose tissue biopsy. All measurements took place within a time window of six weeks. Two days before each of the measurements, participants were instructed to refrain from physical exercise and alcohol consumption. The evening before the measurements, participants consumed a standardized high carbohydrate diner and fasted overnight. For group comparisons, two patient groups were 
included in the study: nine patients with diabetes type II and seven patients with glycogen storage disease type 1a. Patients with diabetes type II were aged 40-75 years, had a BMI between $25-38 \mathrm{~kg} / \mathrm{m}^{2}$ and relatively well controlled diabetes type II: HbA1c $<9.5 \%$. Patients had stable dietary habits, were on treatment with oral medication only (Metformin, Tolbutamide or Gliclazide) and did not use other medication known to interfere with the outcome parameters. Female patients were postmenopausal. Exclusion criteria were engagement in exercise for more than 3 hours per week, uncontrolled hypertension, anemia, unstable body weight (weight loss or gain more than $5 \mathrm{~kg}$ in 3 months preceding enrollment), alcohol or drug abuse, being vegetarian or vegan, having significant food allergies, contraindication for MRI, use of anti-coagulants and clinically relevant active disease. GSD1a patients were recruited aged 18 years and older, clinically diagnosed with GSD1a and without contra-indications for MRI. In vivo validation in adipose tissue was performed in a subgroup of the healthy overweight/obese participants $(n=8)$. Reproducibility measurements in the liver were performed in a separate group of individuals aged between 25-71 years and BMI between $25.7-38.5 \mathrm{~kg} / \mathrm{m}^{2}(\mathrm{n}=7)$.

\section{Overview of specified outcomes}

The primary outcome was lipid composition (SFA fraction) as measured by Magnetic Resonance Spectroscopy and rates of de novo lipogenesis as measured by incorporation of deuterated water. Secondary outcome was hepatic insulin sensitivity as determined by suppression of hepatic glucose output during the low insulin phase in a 2-step hyperinsulinemic euglycemic clamp.

\section{Measurement of lipid content and lipid composition}

In this study, the lipid content and lipid composition (fraction of hepatic SFA, MUFA and PUFA) were determined by proton magnetic resonance spectroscopy ( ${ }^{1} \mathrm{H}-\mathrm{MRS}$ ) (healthy overweight/obese controls without NAFL: $n=7$ for total liver fat content, $n=6$ for liver fat composition, healthy overweight/obese with NAFL: $n=15$, patients with diabetes type II: $n=9$ and patients with GSD type 1a: $\mathrm{n}=7$ ). All ${ }^{1} \mathrm{H}-\mathrm{MRS}$ experiments were performed on a 3T MR system (Achieva 3T-X Philips Healthcare, Best, Netherlands) by using a 32-channel sense cardiac/torso coil (Philips Healthcare, Best, Netherlands). All spectra were obtained by using a STEAM sequence (20) with the following parameters; repetition time (TR) $4500 \mathrm{~ms} / \mathrm{echo}$ time (TE) $20 \mathrm{~ms} /$ mixing time (TM) $16 \mathrm{~ms}$, spectral bandwidth $2000 \mathrm{~Hz}$ and data points 2048. For the in vivo hepatic lipid spectra VAPOR water suppression (21) was applied and an 


\section{$\underline{\text { CHAPTER } 3}$}

additional water reference scan was obtained. The number of averages was 16 for the phantom experiments and in adipose tissue and 128 for the in vivo hepatic spectra. We used a voxel size of $30 \times 30 \times 30 \mathrm{~mm}$ for the hepatic and $15 \times 15 \times 15 \mathrm{~mm}$ for the adipose tissue measurements. All obtained lipid spectra were post-processed in a home-written MATLAB (MATLAB 2014b, The MathWorks, Inc., Natick, Massachusetts, United States.) script in which, prior to fitting, all spectra are individually corrected for phase and frequency shift. Additionally, we performed eddy current correction for the individual lipid spectra. Phasing, frequency alignment and eddy current correction were all performed on individual spectra before signal averaging. Bad quality spectra from in vivo (e.g., due to motion) were removed automatically. For this, the linewidth, amplitude and frequency offset of the peaks in each individual spectrum were compared to the average values of all spectra.

For the setup of the post-processing routine, we used a four-step approach. First, we acquired both ${ }^{1} \mathrm{H}$ and ${ }^{13} \mathrm{C}$ high-resolution (HR) NMR spectra from five different oils (olive, arachis, sunflower, safflower and rice oil), by using a pulse-acquire sequence. The lipid composition of the different oils was determined by integration of the peaks in the methyl region in the ${ }^{13} \mathrm{C} \mathrm{HR}$ spectra. In the ${ }^{1} \mathrm{H}$ HR spectra four different regions were integrated, corresponding to the methyl protons (around $0.90 \mathrm{ppm}$ ), the allylic protons (around $2.02 \mathrm{ppm}$ ), the alpha carbonyl group (around $2.20 \mathrm{ppm}$ ) and the diallylic protons (around $2.75 \mathrm{ppm}$ ) (supplementary figure 1). The ${ }^{1} \mathrm{H}$ HR spectra were used to develop a basis set used by the developed fitting algorithm. The lipid signal was described by 13 individual resonances ( 2 for the methyl group, 5 for the methylene group, 1 for the beta methyle-group, 2 for the allylic group, 2 for the alpha-carbonyl group and 1 for the diallylic group). In this basis set, the relative frequency shifts, the splitting patterns and the initial linewidths were described.

In a second setup step, we acquired spectra from the different oils on our clinical $3 \mathrm{~T}$ scanner. Eddy current correction was applied based on an additional water reference scan with an identical experimental setup. The oil spectra were fitted with the developed Matlab algorithm. To this end, using the basis set from the HR spectra, the time domain signal was simulated. The amplitudes and relative frequency shifts of the individual peaks were updated iteratively. In each step the difference between the simulated spectrum and the acquired spectrum was minimized in the frequency domain. Next to these individual parameters, also the Gaussian line broadening, the Lorentzian line broadening, the zero-order phase, an overall frequency shift and the baseline offset were automatically updated, affecting all the peaks in an identical fashion. Essential for our approach, we furthermore fixed the ratio of the methyl group and the 
alpha-carbonyl group (which overlaps with the allylic group at 3T) to 0.6 . Theoretically this factor should be 0.67 , as the methyl group contains 3 protons, and the alpha-carbonyl group contains 2 protons and both groups are present only once in every fatty acid. However, due to TE-induced losses this factor will be affected. Therefore, this factor was determined empirically. In a final fine-tuning step of the fitting routine, the zero-order phase was accurately fitted by minimizing the residual in the spectrum in the methyl and allylic + alpha-carbonyl region specifically.

The signal amplitude ratio of the diallylic over the methyl peaks and the ratio of the alphacarbonyl + allylic over the methyl peaks was determined in both the phantom and the HR experiment, to empirically determine correction factors for TE-induced signal in our STEAM recordings. These empirically determined correction factors were then used for the measurements in adipose tissue and in the liver to calculate the lipid composition using the following formulas:

$$
\begin{array}{cc}
\% P U F A=C A *\left(\frac{2}{3} * \frac{S_{\text {diallylic }}}{S_{\text {methyl }}}\right) * 100 & \text { Eq. } 1 \\
\% M U F A=C B *\left[\frac{3}{4} *\left(\frac{S_{\text {allylic }}+S_{\text {alphacarbonyl }}}{S_{\text {methyl }}}-\frac{2}{3}\right)\right] * 100-\% P U F A & \text { Eq. } 2 \\
\% S F A=100-\% P U F A-\% \text { MUFA } & \text { Eq. } 3
\end{array}
$$

with $\mathrm{CA}$ and $\mathrm{CB}$, the empirically determined correction factor for the PUFA and MUFA calculation respectively $(\mathrm{CA}=0.83$ and $\mathrm{CB}=0.99$; see also supplementary table 1$)$.

In a third step, the lipid composition in subcutaneous adipose tissue was determined in vivo with ${ }^{1} \mathrm{H}-\mathrm{MRS}$ using the developed fitting routine and this was compared to the ex vivo lipid composition as determined by mass spectrometry analysis in adipose tissue biopsies, in eight participants. For the adipose tissue ${ }^{1} \mathrm{H}-\mathrm{MRS}$ measurement, we applied a gradient cycled STEAM sequence, to correct for eddy currents, as previously described (22).

In a final validation step to determine reproducibility, we applied the developed methodology in the liver of seven healthy individuals (BMI $30.6 \pm 3.7 \mathrm{~kg} / \mathrm{m}^{2}$; age $49 \pm 17.2$ year; 3 women), with a wide range of intrahepatic fat (2.1-19.6\%). To this end, we performed the ${ }^{1} \mathrm{H}-\mathrm{MRS}$ acquisition and repeated this after replacement of the subject on the table. The ICC was calculated for the lipid composition and total lipid content as a measure to determine the 


\section{CHAPTER 3}

reproducibility between two consecutive measurements. Lipid content was calculated after $\mathrm{T}_{2}$ correction as ratio of the $\mathrm{CH}_{3}$ peak relative to the unsuppressed water resonance, expressed as percentage weight/weight.

\section{Deuterated water measurement of DNL}

A background blood sample was drawn in the afternoon before intake of the deuterated water in 18 healthy overweight/obese participants. Together with the evening meal, participants were given $2.86 \mathrm{~g} / \mathrm{kg}$ body weight deuterated water $(70 \% 2 \mathrm{H} 2 \mathrm{O}$, Cambridge Isotope laboratories) in two servings. A blood sample for DNL analysis was drawn at fasting in the morning, 16 hours after the first serving of deuterated water. The DNL was analyzed from enrichment in VLDLTG of deuterated water $(10,23)$. Two participants were excluded from the analysis of DNL because of TG levels higher than $4 \mathrm{mmol} / \mathrm{L}$, influencing the reliability of the deuterated water measurement.

\section{Body composition}

Body mass and body volume were assessed using air-displacement plethysmography (ADP) using the Bod Pod device (Cosmed, Italy, Rome) according to the manufacturer's instructions on the day of the hyperinsulinemic-euglycemic clamp (healthy overweight/obese volunteers: $\mathrm{n}=21$, patients with diabetes type II: $\mathrm{n}=9$ ) (24). Thoracic gas volume was predicted based on equations included in the Bod Pod software (version 4.2.0). From these data, body fat percentage was calculated as described by Siri (25).

\section{Subcutaneous adipose tissue biopsy}

In a subgroup of eight healthy overweight/obese participants, a subcutaneous adipose tissue biopsy $(\sim 1 \mathrm{~g})$ was collected $6-8 \mathrm{~cm}$ lateral from the umbilicus, under local anesthesia $(1 \%$ lidocaine) by needle biopsy before the start of the hyperinsulinemic-euglycemic clamp. Upon tissue collection, the tissue was rinsed with sterile saline and visible blood vessels were removed. Thereafter, the biopsy was snap-frozen in liquid nitrogen and stored at $80^{\circ} \mathrm{C}$ for later lipid composition analyses.

\section{Analysis of VLDL and adipose tissue triglycerides}

Plasma samples used for VLDL-TG analyses were taken around $16 \mathrm{pm}$ before the intake of the deuterated water. The VLDL fractions (20-50 $\mu \mathrm{l})$ and adipose tissues $(10-20 \mathrm{mg})$ were extracted using the BUME method $(26,27)$. The total lipid extracts were evaporated under a stream of 
nitrogen and reconstituted in $250 \mu \mathrm{L}$ chloroform/methanol [2:1]. Triglycerides were detected by direct infusion (shotgun) analysis on a QTRAP 5500 mass spectrometer (Sciex, Concord, Canada) equipped with a robotic nanoflow ion source, TriVersa NanoMate (Advion BioSciences, Ithaca, NJ) according to previous work (28). More specifically, a fraction of the total lipid extract was diluted 1:100 (for VLDL) and 1:100 000 (for adipose tissue) in chloroform: methanol [1:2] with $5 \mathrm{mM}$ ammonium acetate and infused at $250 \mathrm{nl} / \mathrm{min}$ for 15 minutes. The NanoMate was run with a voltage of $1.2 \mathrm{kV}$ and a gas pressure of $0.8 \mathrm{psi}$. The analysis was performed in positive ion mode by neutral loss detection of 11 common acyl fragments formed during collision induced dissociation of the ammoniated triglycerides. The nano-interface of the mass spectrometer was heated to 60 degrees and the measurements was made using a scan speed of 200 dalton/sec. The neutral loss scans were cycled and in total 50 cycles were acquired for each neutral loss scan (one neutral loss for each fatty acid). The data was processed using the LipidView 1.2 software (Sciex, Concord, Canada) and the most abundant signal intensities from the most commonly found triglyceride species were used to calculate the abundance and composition of the different triglycerides (supplementary table 2 and 3). Quantification was made using a one-point calibration against glyceryl- $\mathrm{d}_{5}$ hexadecanoate (CDN isotopes, Quebec, Canada), which was added to the infusion solvent.

\section{Hyperinsulinemic-euglycemic clamp}

A two-step hyperinsulinemic-euglycemic clamp was performed to assess hepatic insulin sensitivity using the low-dose phase $\left(10 \mathrm{mU} / \mathrm{m}^{2} / \mathrm{min}\right)$ (healthy overweight/obese participants: $\mathrm{n}=21)$. A primed continuous infusion of $\mathrm{D}-[6,6-2 \mathrm{H} 2] \mathrm{glucose}(0.04 \mathrm{mg} / \mathrm{kg} / \mathrm{min})$ was started to determine rates of endogenous glucose production (EGP), glucose appearance (Ra), and glucose disposal (Rd). After $180 \mathrm{~min}$, participants were given infusion of low insulin (10 $\mathrm{mU} / \mathrm{m} 2 / \mathrm{min}$ ) for $3 \mathrm{~h}$. During the last $30 \mathrm{~min}$ of the insulin infusion step, blood samples were collected. Steele's single pool non- steady state equations were used to calculate glucose Ra and $\mathrm{Rd}(29)$. Volume of distribution was assumed to be $0.160 \mathrm{~L} / \mathrm{kg}$ for glucose. Hepatic insulin sensitivity was calculated as the percentage of EGP suppression during the low-dose phase. In addition, hepatic insulin sensitivity was determined according to this methodology in patients with diabetes type II $(n=9)$. Clamp parameters are shown in supplementary table 4. 


\section{Statistical analysis}

Results are expressed as mean \pm SEM for group comparisons. Population characteristics are expressed as mean $\pm \mathrm{SD}$. Continuous variables were tested for normality. Two-sided pearson correlation was performed to identify correlations between variables. For non-normally distributed data two-sided spearman correlation was performed. Group comparisons were assessed by one-way ANOVA. For non-normally distributed data Kruskal-Wallis analyses was performed to compare groups. In case of significant group differences in the group comparisons, post-hoc analyses were performed using Bonferroni correction to test which groups were significantly different. A p-value $<0.05$ was considered statistically significant. Statistical analyses were performed using SPSS 23.0 for Mac OS.

\section{RESULTS}

Table 1: subject characteristics of control and NAFL participants, patients with T2D and GSD1a.

\begin{tabular}{l|c|c|c|c}
\hline & $\begin{array}{l}\text { Control } \\
(\mathrm{n}=7)\end{array}$ & $\begin{array}{l}\text { NAFL } \\
(\mathrm{n}=15)\end{array}$ & T2D (n=9) & GSD1a (n=7) \\
\hline Age (years) & $59 \pm 6.8$ & $58 \pm 7.1$ & $65 \pm 4.5$ & $37 \pm 12.3^{\mathrm{A}, \mathrm{B}, \mathrm{C}}$ \\
BMI (kg/m²) & $29.1 \pm 2.3$ & $30.7 \pm 3.1$ & $29.4 \pm 4.2$ & $27.5 \pm 3.2$ \\
Sex (f/m) & $6 / 1$ & $7 / 8$ & $2 / 7$ & $5 / 2$ \\
Body fat (\%) & $42.1 \pm 8.1$ & $42.1 \pm 7.8$ & $34.1 \pm 5.8$ & - \\
Plasma glucose (mmol/L) & $5.2 \pm 0.4$ & $5.6 \pm 0.5$ & $7.5 \pm 1.1^{\mathrm{A}, \mathrm{B}}$ & $3.9 \pm 0.8^{\mathrm{A}, \mathrm{B}, \mathrm{C}}$ \\
Plasma insulin (pmol/L) & $35.2 \pm 8.1$ & $89.8 \pm 40.4^{\mathrm{A}}$ & $72.8 \pm 58.3$ & $13.8 \pm 5.0^{\mathrm{B}, \mathrm{C}}$ \\
Plasma NEFA (mmol/L) & $667 \pm 58$ & $623 \pm 141$ & $566 \pm 211$ & $876 \pm 421$ \\
Plasma TG (mmol/L) & $1.5 \pm 0.8$ & $2.6 \pm 1.2$ & $1.6 \pm 0.5$ & $5.0 \pm 1.5^{\mathrm{A}, \mathrm{B}, \mathrm{C}}$ \\
ALT (U/L) & $22 \pm 4.1$ & $35 \pm 14.4$ & $27 \pm 11.4$ & $18 \pm 4.9^{\mathrm{B}}$ \\
AST (U/L) & $22 \pm 3.7$ & $28 \pm 6.3$ & $23 \pm 5.9$ & $34 \pm 9.3^{\mathrm{A}, \mathrm{C}}$ \\
Intrahepatic fat content & $2.2 \pm 1.3$ & $14.4 \pm 10.4^{\mathrm{A}}$ & $6.9 \pm 5.2$ & $16.5 \pm 18.9^{\mathrm{A}}$ \\
(\% weight/weight) & & & & \\
\hline
\end{tabular}

Data are presented as mean \pm SD. Overweight/obese controls without NAFL (controls, $\mathrm{n}=7$ ), overweight/obese with NAFL (NAFL, $n=15$ ), patients with diabetes type II (T2D, n=9) and GSD type 1a (GSD1a, $\mathrm{n}=7$ ). Age was significantly lower in GSD1a compared to control $\left(\mathrm{p}=2.5 \times 10^{-5}\right)$, NAFL $\left(\mathrm{p}=3.0 \times 10^{-6}\right)$ and T2D $\left(\mathrm{p}=1.47 \times 10^{-7}\right)$. Plasma glucose was significantly lower in GSD1a compared to control $(\mathrm{p}=0.011)$, NAFL $\left(\mathrm{p}=5.6 \times 10^{-5}\right)$ and T2D $\left(\mathrm{p}=1.44 \times 10^{-10}\right)$ and was significantly higher in T2D compared to control $\left(\mathrm{p}=4.0 \times 10^{-6}\right)$ and NAFL $\left(\mathrm{p}=6.0 \times 10^{-6}\right)$. Plasma insulin was significantly lower in GSD1a compared to NAFL $\left(\mathrm{p}=3.4 \times 10^{-5}\right)$ and T2D $(\mathrm{p}=0.006)$ and was significantly higher in NAFL compared to control $(\mathrm{p}=0.048)$. Plasma TG was significantly higher in GSD1a compared to control $\left(\mathrm{p}=3.0 \times 10^{-6}\right)$, NAFL $\left(\mathrm{p}=1.33 \times 10^{-4}\right)$ and T2D $\left(\mathrm{p}=2.0 \times 10^{-6}\right)$. ALT was significantly higher in NAFL compared to GSD1a ( $p=0.011$ ). AST was significantly higher in GSD1 a compared to control $(p=0.006)$ and T2D $(\mathrm{p}=0.017)$. Intrahepatic fat content was significantly higher in NAFL compared to control $(p=0.002)$ and GSD1a compared to control $(p=0.027)$. A Significantly different from control, ${ }^{B}$ significantly different from NAFL, ${ }^{\mathrm{C}}$ significantly different from T2D (Kruskal-Wallis, $\mathrm{p}<0.05$ for plasma insulin, plasma NEFA, ALT and intrahepatic fat content, and one-way ANOVA, $\mathrm{p}<0.05$ for all other parameters). Bonferroni correction was used for post-hoc analyses. 


\section{Development and validation of hepatic ${ }^{1} \mathrm{H}-\mathrm{MRS}$ method}

To allow determination of hepatic fatty acid composition, we developed a ${ }^{1} \mathrm{H}-\mathrm{MRS}$ analysis protocol. To this end, we used information from ${ }^{1} \mathrm{H}$ high-resolution (HR) NMR spectra from five different vegetable oils to improve our analysis routine and used ${ }^{13} \mathrm{C}$ HR NMR to determine the true lipid composition of these oils. We acquired spectra from these different oils with proton magnetic resonance spectroscopy $\left({ }^{1} \mathrm{H}-\mathrm{MRS}\right)$ on our clinical 3T scanner and calculated average corrections factors to correct for TE-induced losses in the ${ }^{1} \mathrm{H}-\mathrm{MRS}$ spectra. When using the average corrections factors, the lipid composition as determined by our clinical protocol showed excellent agreement with the true lipid composition determined by highresolution NMR: the intraclass correlation coefficient (ICC) for SFA, MUFA and PUFA fraction was $0.982,0.970,0.987$ and the $\mathrm{CV}$ was $6 \%, 9 \%$ and $9 \%$, respectively (figure 1 ). 


\section{$\underline{\text { CHAPTER } 3}$}

A)
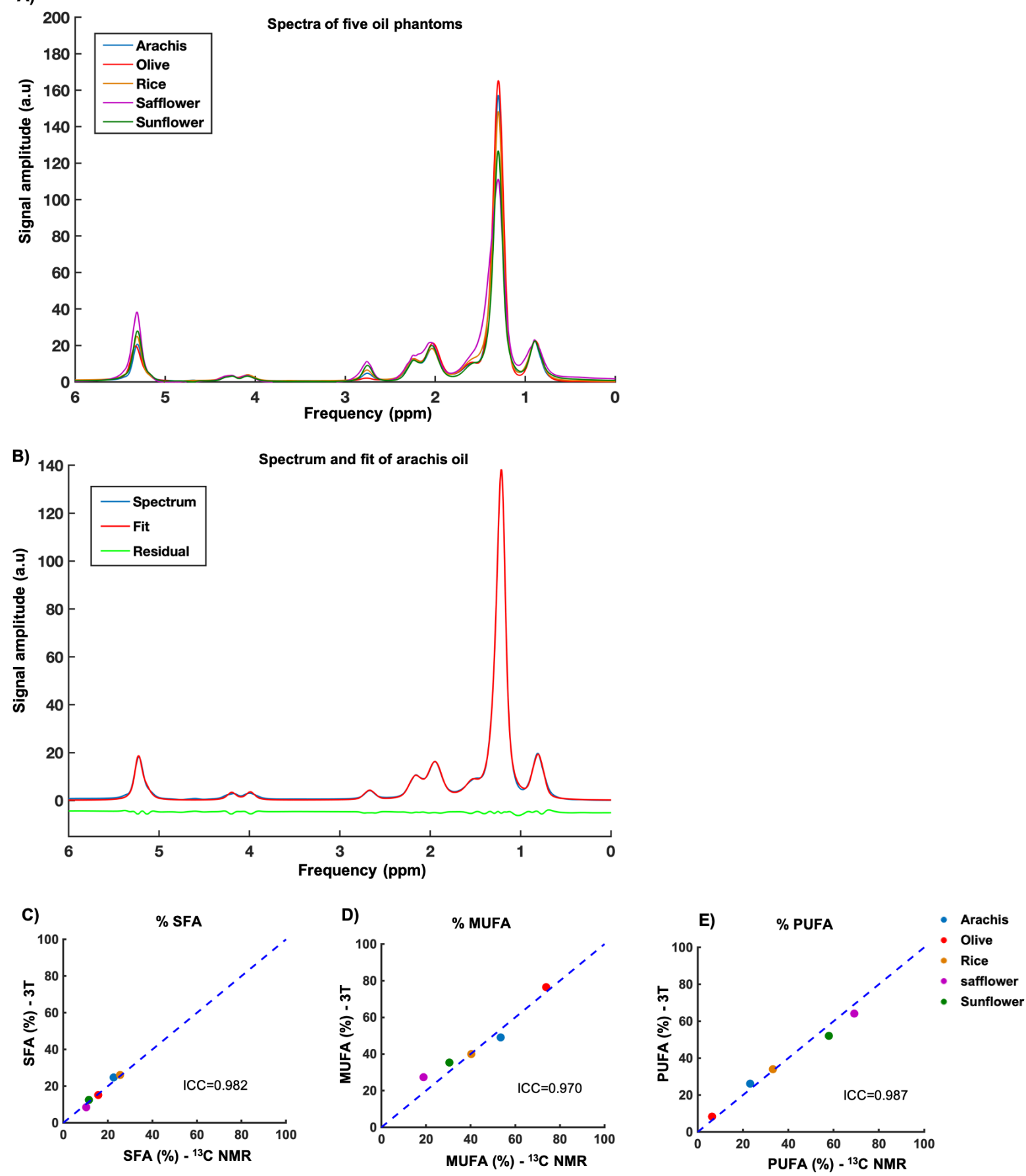

Figure 1: Validation of ${ }^{1}$ H-MRS method in oil phantoms. (A) Lipid spectra acquired from five different oil phantoms (arachis, olive, rice, safflower and sunflower oil) showing the different lipid proton peaks and their position. (B) Lipid spectrum and fit of arachis oil. Correlations between (C) SFA, (D) MUFA, (E) PUFA measured at $3 \mathrm{~T}$ with ${ }^{1} \mathrm{H}$-MRS and measured with high-resolution ${ }^{13} \mathrm{C}-\mathrm{NMR}$ spectroscopy. The intraclass correlation coefficient (ICC) is shown in the respective plots (Intraclass correlation).

As it is known that in vivo several factors can influence MR spectra, the next step was to validate our method in vivo. To this end, we aimed to validate our ${ }^{1} \mathrm{H}-\mathrm{MRS}$ lipid composition measurement with analysis performed in biopsy material. As it is ethically difficult to take liver 
biopsies for this purpose, we rather performed subcutaneous adipose tissue biopsies, which is far less invasive. Therefore, we validated our MRS method in vivo by comparing lipid composition in subcutaneous adipose tissue acquired by the ${ }^{1} \mathrm{H}-\mathrm{MRS}$ technique and by mass spectrometry analysis in subcutaneous adipose tissue biopsies in eight participants (figure 2). As can be seen in figure 2, there was reasonably good agreement between the two methods. The ICC for SFA, MUFA and PUFA fraction was $0.333,0.146,0.306$ and the CV was $8 \%$, $7 \%, 12 \%$, respectively. Thus, lipid composition determined by ${ }^{1} \mathrm{H}-\mathrm{MRS}$ in vivo is in close agreement with lipid composition measured ex vivo in adipose tissue biopsies.

A)
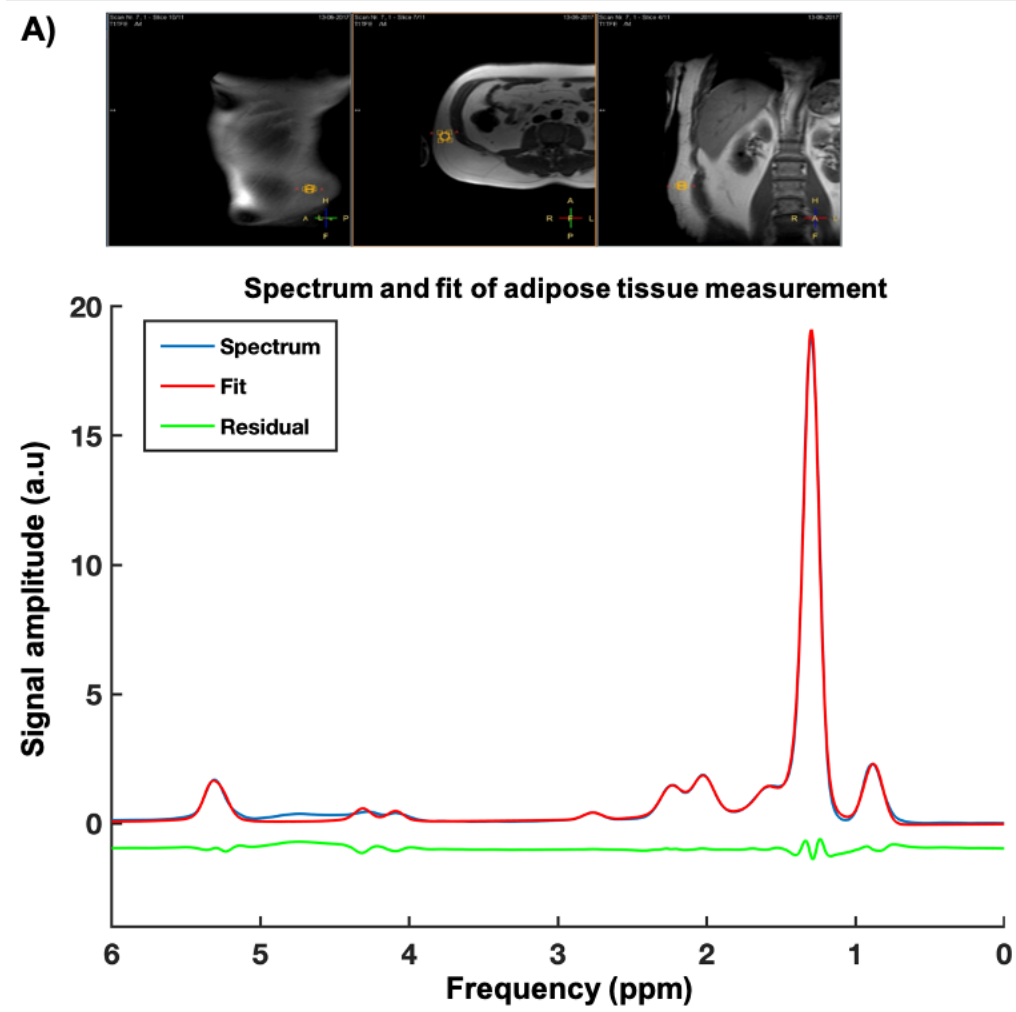

B)

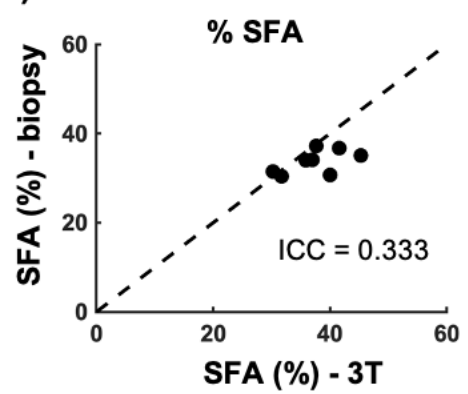

C)

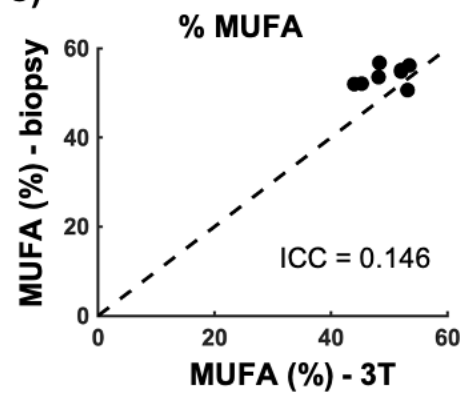

D)

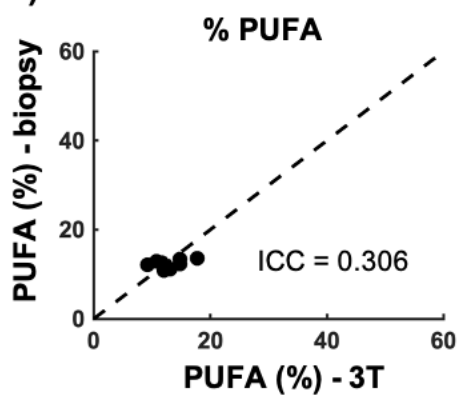

Figure 2: Validation of ${ }^{1}$ H-MRS method in subcutaneous adipose tissue. (A) $\mathrm{T}_{2}$ weighted Turbo spin echo MR image showing the voxel position located on adipose tissue and its corresponding lipid spectrum together with the fitted spectrum. The relationships between subcutaneous adipose tissue measured at $3 \mathrm{~T}$ and adipose lipid composition determined through biopsy for the different lipid fractions: (B) SFA, (C) MUFA and (D) PUFA ( $\mathrm{n}=8$ ). The intraclass correlation coefficient (ICC) is shown in the respective plots (Intraclass correlation). 


\section{$\underline{\text { CHAPTER } 3}$}

A)
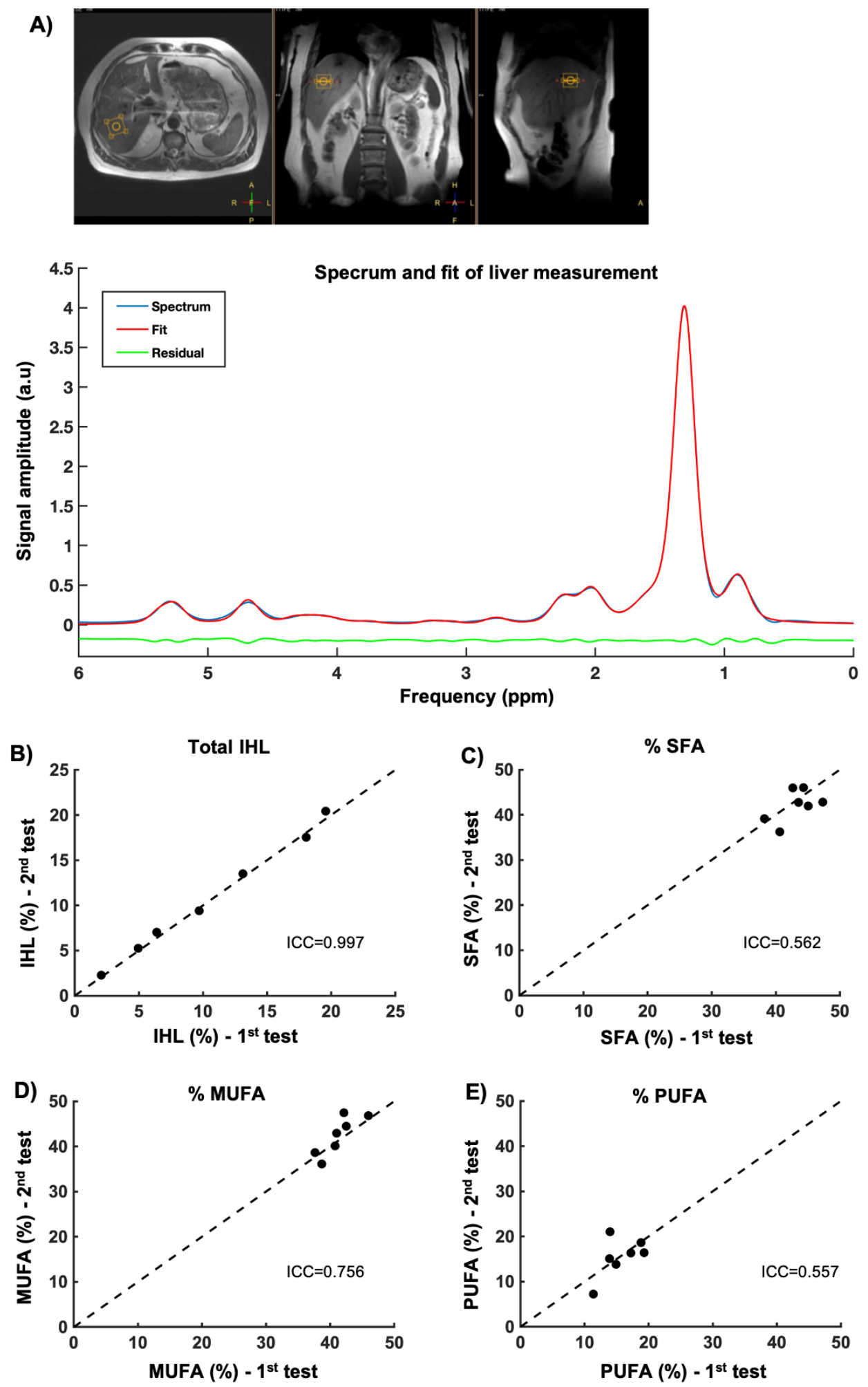

Figure 3: Validation of ${ }^{1}$ H-MRS method by testing reproducibility in vivo. (A) $T_{2}$ weighted Turbo spin echo MR image showing the voxel position located on liver and its corresponding lipid spectrum together with the fitted spectrum. Scatter plots showing the reproducibility of (B) total liver fat content and (C) SFA fraction, (D) MUFA fraction and (E) PUFA fraction. ( $n=7)$. Reproducibility was tested by performing two repeated measurements. The intraclass correlation coefficient (ICC) is shown in the respective plots (Intraclass correlation). 
The final validation step was to apply our protocol in the liver in vivo. We tested reproducibility in seven healthy individuals (separate group) with intrahepatic lipid content ranging from 2$18 \%$ (figure 3). As can be seen in figure 3, the ICC for total IHL content, SFA, MUFA and PUFA fraction was found to be $0.997,0.562,0.756,0.557$ and the CV was $4 \%, 4 \%, 3 \%$, and $12 \%$, respectively. These results indicate high reproducibility for determining in vivo hepatic lipid composition using our developed approach. In addition, we compared hepatic lipid composition by our MRS method to plasma VLDL-triglyceride (VLDL-TG) composition acquired by mass spectrometry analysis in seventeen participants (figure 4). As can be seen in figure 4, especially the SFA fraction showed a strong correlation with hepatic SFA\% (Pearson $\mathrm{r}=0.80, \mathrm{p}<0.001)$.
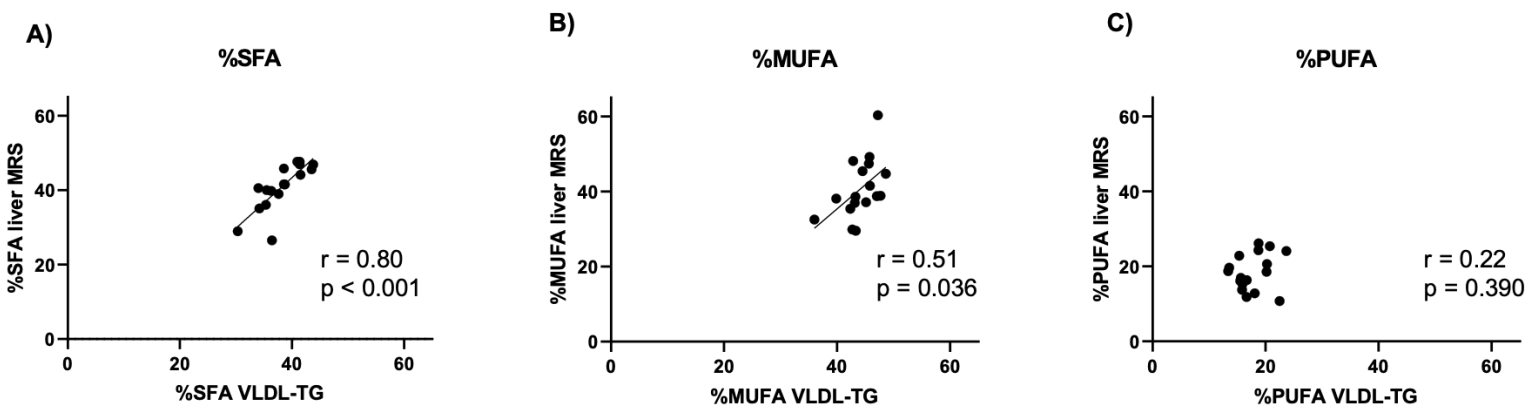

Figure 4: The relationship between hepatic lipid composition and plasma VLDL-TG composition. Relationships are shown for the different lipid fractions: (A) SFA, (B) MUFA and (C) PUFA ( $\mathrm{n}=17)$. Hepatic \%SFA determined with MRS and \%SFA in VLDL-TG correlated significantly $\left(\mathrm{p}=1.38 \times 10^{-4}\right)$. The correlation coefficient is shown in the respective plots (two-sided Pearson correlation).

\section{Higher hepatic SFA is associated with increased DNL}

DNL is an important factor in the development of fatty liver (30). We hypothesized that DNL would specifically lead to the accumulation of saturated fatty acids, as palmitate is the main product of DNL. Therefore, we determined DNL by deuterated water in overweight and obese participants with a wide range of liver fat content $(n=16,0.9-38.4 \%)$ and related it to the hepatic fatty acid composition determined by our MRS protocol in the same volunteers. DNL was not associated with total liver fat content (figure 5A). Interestingly, however, DNL correlated positively with the hepatic SFA fraction (Pearson $r=0.52, p=0.047$; figure 5B). Furthermore, we found a strong negative correlation between DNL and hepatic MUFA fraction (Pearson $\mathrm{r}=-$ 0.71, $\mathrm{p}=0.003$; figure 5C). In addition, DNL was negatively correlated with MUFA/SFA ratio (Pearson $r=-0.64 \mathrm{p}=0.010$; figure 5E). No association was found between DNL and PUFA fraction (figure 5D). Of note, SFA fraction in plasma VLDL-TG did not correlate with DNL (Pearson $\mathrm{r}=0.27, \mathrm{p}=0.308$ ). These results suggest that 1 ) higher rates of DNL lead to altered 


\section{$\underline{\text { CHAPTER } 3}$}

saturation of hepatic lipids, and 2) that our non-invasive method can be used as a measure of the degree of DNL, directly in the liver.

A)

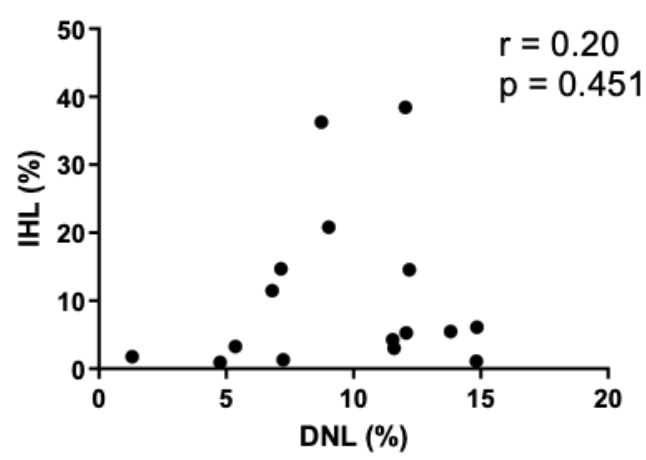

C)

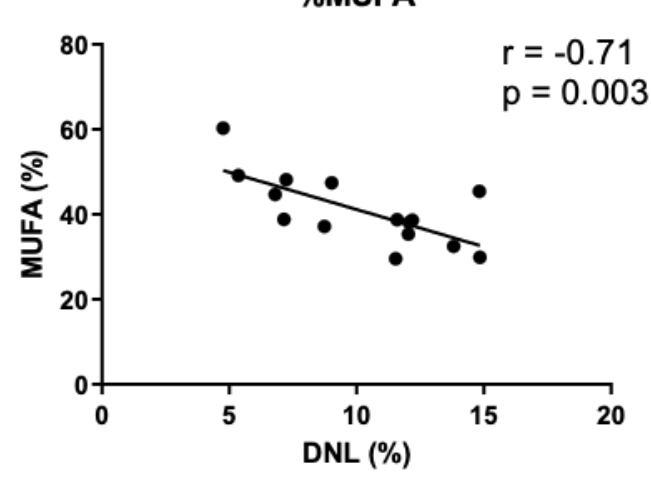

E)

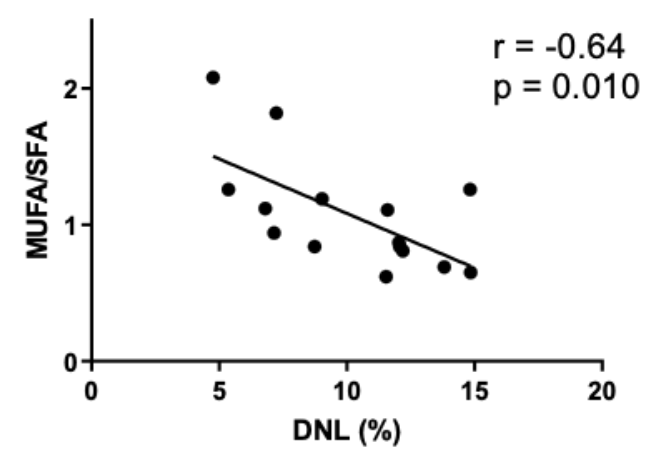

B)

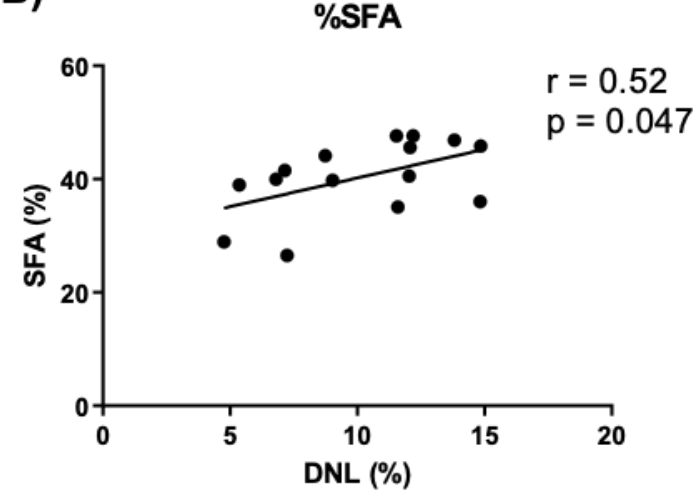

D)

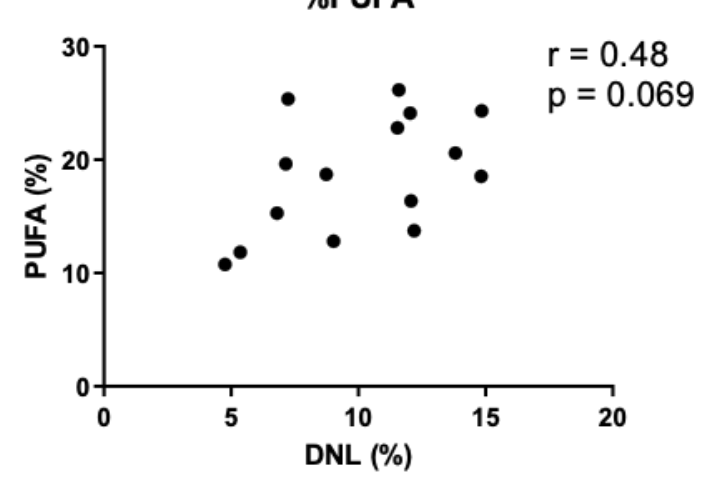

Figure 5: Relationship between DNL and liver fat composition. The relationships between DNL and (A) total liver fat content, (B) SFA fraction, (C) MUFA fraction, (D) PUFA fraction and (E) MUFA/SFA ratio in healthy overweight/obese participants (with and without NAFL, $n=16$ for total liver fat content and $n=15$ for the fatty acid fractions and MUFA/SFA ratio). The correlation coefficient is shown in the respective plots (A; two-sided Spearman correlation, B-E; two-sided Pearson correlation). 


\section{Hepatic SFA fraction is of clinical relevance in humans}

To investigate if hepatic lipid composition may also have clinical relevance, we compared hepatic lipid composition in volunteers with a range in metabolic complications.

A)

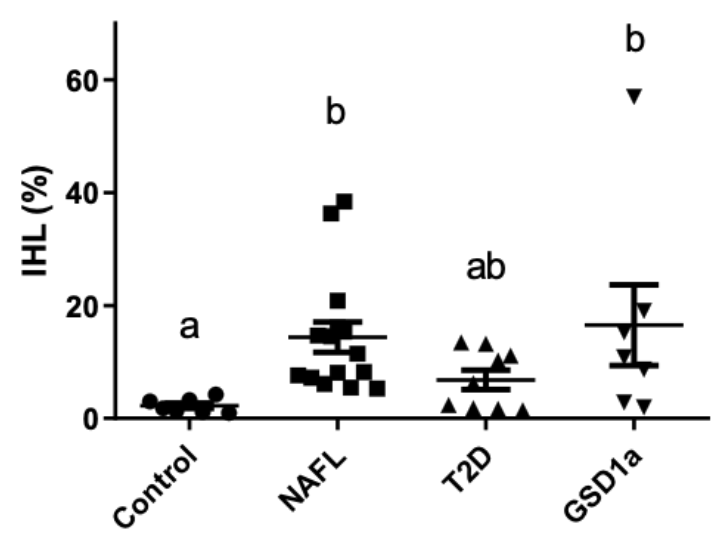

C)

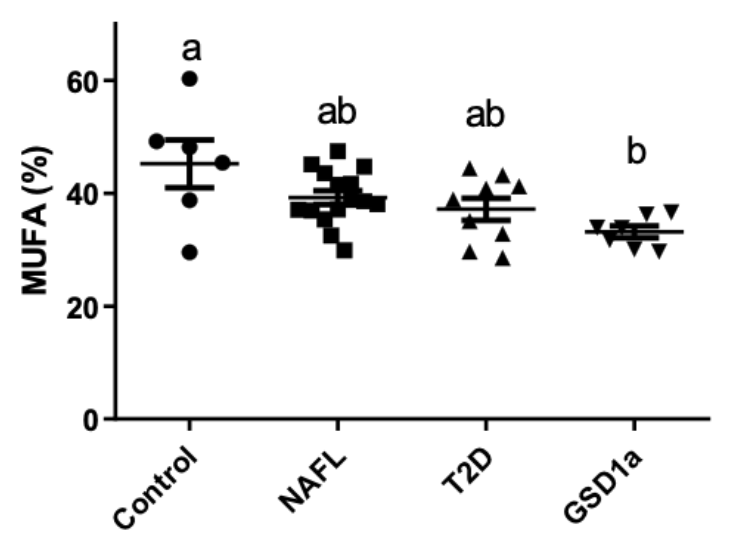

B)

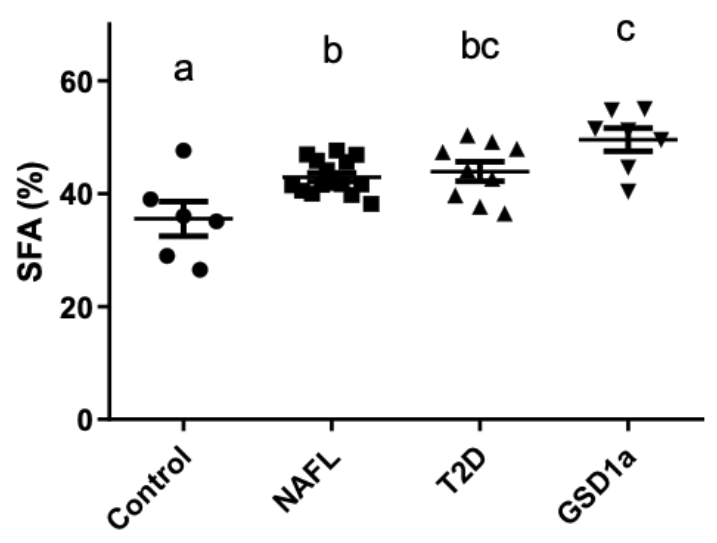

D)

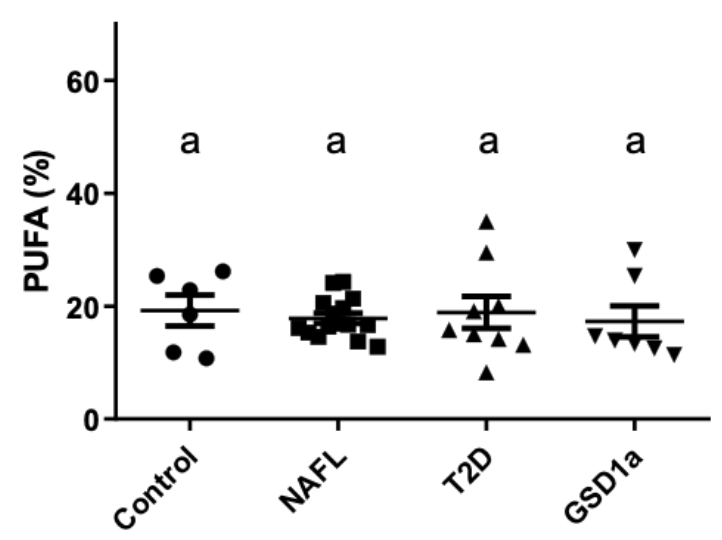

Figure 6: Liver fat content and composition in groups with different metabolic disorders. Comparisons between overweight/obese controls without NAFL (controls, $n=7$ for total liver fat content, $n=6$ for liver fat composition), overweight/obese with NAFL (NAFL, $n=15$ ), patients with diabetes type II (T2D, n=9) and GSD type 1a (GSD1a, n=7). (A) Total liver fat content in control, NAFL, T2D and GSD1a. Total liver fat content was significantly higher in the NAFL group compared to the control group ( $p=0.002$ ) and in the GSD1a group compared to the control group $(p=0.027)$. (B) SFA fraction in control, NAFL, T2D and GSD1a. SFA fraction was significantly higher in the GSD1a group compared to the control group $\left(\mathrm{p}=7.3 \times 10^{-5}\right)$ and NAFL group $(\mathrm{p}=0.034)$, significantly higher in the T2D group compared to the control group $(\mathrm{p}=0.016)$, and significantly higher in the NAFL group compared to the control group ( $\mathrm{p}=0.022$ ). (C) MUFA fraction in control, NAFL, T2D and GSD1a. MUFA fraction was significantly lower in the GSD1a group compared to the control group ( $p=0.006$ ). (D) PUFA fraction in control, NAFL, T2D and GSD1a. Data are presented as mean with error bars showing the SEM. Different letters indicate significant differences between groups (Kruskal-Wallis, $\mathrm{p}<0.05$ for IHL and PUFA, and one-way ANOVA, $\mathrm{p}<0.05$ for SFA and MUFA). Bonferroni correction was used for post-hoc analyses. 


\section{$\underline{\text { CHAPTER } 3}$}

We subdivided the volunteers in whom we demonstrated the relationship between DNL and hepatic SFA, into participants with NAFL (liver fat content $>5 \%, n=15$ ) and control participants (liver fat content $<5 \%, n=7$ ); volunteers with NAFL are known to be at increased risk to develop diabetes type II and other cardiometabolic disorders (6-8). In addition, we measured hepatic lipid fractions and hepatic insulin sensitivity (IS) in a group of patients with diabetes type II $(n=9)$ and furthermore, determined hepatic lipid fractions in glycogen storage disease type 1a (GSD1a) patients $(n=7)$, known to have elevated rates of DNL. This inborn error of metabolism is caused by a defect in glucose-6-phosphatase, which hampers the final step in gluconeogenesis and glycogenolysis in liver and kidney, and, as a consequence, favours the shift of glucose-6-phosphate towards DNL and hepatic steatosis (31). Control and NAFL participants and patients with diabetes type II were comparable in age and BMI. All subject characteristics are summarized in Table 1.

Hepatic SFA fraction was higher in NAFL individuals $(42.9 \% \pm 0.8 \%$, ANOVA, $\mathrm{p}=0.022$, mean \pm SEM; figure $6 \mathrm{~B})$ and in diabetes type II patients $(43.9 \% \pm 1.7 \%$, ANOVA, $p=0.016$; figure 6B) compared to controls $(35.5 \% \pm 3.1 \%)$. Hepatic MUFA and PUFA fractions did not significantly differ between these groups (figure 6C and 6D). No significant associations were found between hepatic fat content and any of the FA fractions in healthy participants with and without NAFL. Therefore, these data suggest that specifically the SFA fraction is elevated in metabolically compromised volunteers.

To further confirm that DNL and SFA fraction may be causally related, and to further investigate the clinical relevance of SFA, we determined SFA fraction in a specific group of patients, known to have elevated rates of DNL, i.e., patients with glycogen storage disease type 1a (GSD1a) $(n=7)$. In these patients, SFA fraction $(49.5 \% \pm 2.0 \%)$ was markedly higher compared to NAFL participants $(42.9 \% \pm 0.8 \%$, ANOVA, $\mathrm{p}=0.034$; figure $6 \mathrm{~B})$ and controls $(35.5 \% \pm 3.1 \%$, ANOVA, $\mathrm{p}<0.001$; figure $6 \mathrm{~B})$. The MUFA fraction in these patients was reduced compared to controls $(33.2 \% \pm 1.1 \%$ vs. $45.2 \% \pm 4.2 \%$, ANOVA, $p=0.006$; figure 6C). These results further confirm that the fraction of hepatic SFA and MUFA reflect the rate of DNL and can be used as a non-invasive measure to characterise hepatic metabolism in clinical patients in more detail. 


\section{Higher hepatic SFA is associated with reduced hepatic IS}

Our results so far suggest that specifically the saturated fat fraction is elevated in patients and individuals with an enhanced metabolic risk.
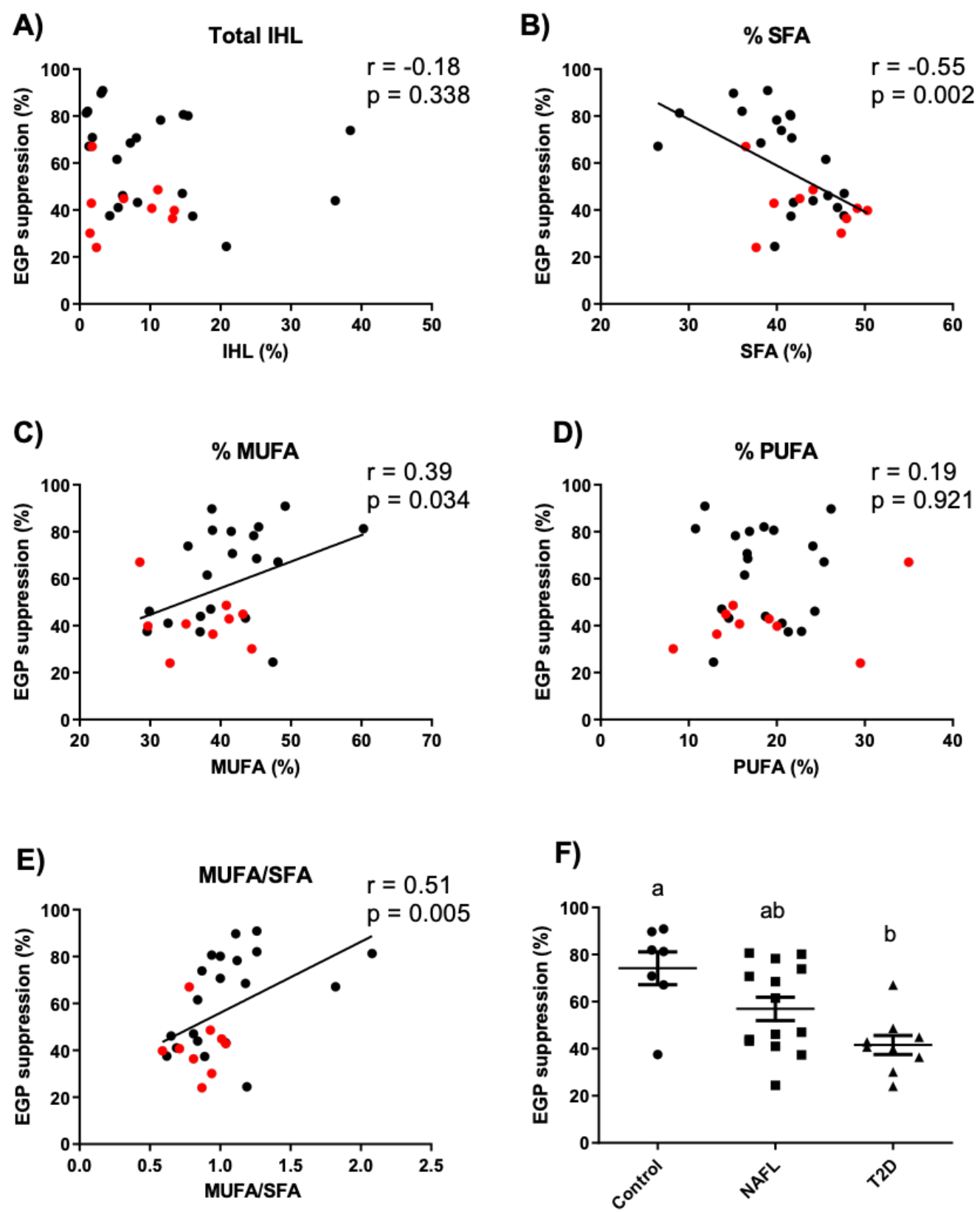

Figure 7: The relationship between liver fat composition and hepatic insulin sensitivity. The relationship between hepatic IS (EGP suppression) and (A) total liver fat content, (B) SFA fraction, (C) MUFA fraction and (D) PUFA fraction and (E) MUFA/SFA ratio in overweight and obese individuals (healthy with and without NAFL in black and patients with T2D in red, $n=30$ for total liver fat content and $n=29$ for the fatty acid fractions and MUFA/SFA ratio). The correlation coefficient is shown in the respective plots (Two-sided Spearman correlation). (F) EGP suppression in healthy overweight/obese without NAFL (controls, $n=7)$, overweight/obese with NAFL $(n=14)$ and patients with T2D $(n=9)$. EGP suppression in patients with T2D was significantly lower compared to controls $(\mathrm{p}=0.002)$. Data are presented as mean with error bars showing the SEM. Different letters indicate significant differences between groups (one-way ANOVA, $\mathrm{p}<0.05$ ). Bonferroni correction was used for post-hoc analyses. 


\section{$\underline{\text { CHAPTER } 3}$}

These data are consistent with findings in animal studies, where it was found that specifically hepatic SFA is of importance in relation to insulin resistance (13). We therefore investigated the relationship between hepatic lipid composition and hepatic IS using the golden-standard two-step hyperinsulinemic euglycemic clamp. Hepatic IS was found to be $40 \%$ lower in patients with diabetes type II compared to controls (EGP suppression controls: $74.2 \% \pm 7.0 \%$ and T2D: $41.6 \% \pm 4.1 \%$, ANOVA, $\mathrm{p}=0.002$; figure $7 \mathrm{~F}$ ), with intermediate values in NAFL (EGP suppression $56.9 \% \pm 4.9 \%$; figure $7 \mathrm{~F}$ ). The hepatic SFA fraction was strongly and negatively associated with hepatic IS (Spearman $\mathrm{r}=-0.55, \mathrm{p}=0.002$; figure $7 \mathrm{~B}$ ), whereas correlation with hepatic MUFA fraction was weaker (Spearman $\mathrm{r}=0.39, \mathrm{p}=0.034$; figure $7 \mathrm{C}$ ) and PUFA fraction and total hepatic fat content did not correlate with hepatic IS (figure 7A, D). Furthermore, the MUFA/SFA ratio was positively associated with hepatic IS (Spearman $\mathrm{r}=0.51, \mathrm{p}=0.005$; figure $7 \mathrm{E}$ ). These results suggest that specifically the SFA fraction in the liver negatively contributes to hepatic IS, rather than the total amount of hepatic fat.

\section{DISCUSSION}

The relation of hepatic lipid composition to cardiometabolic health and specifically insulin resistance has not been investigated in humans. Due to the invasive procedures necessary and due to challenges accompanying the use of non-invasive methods such as ${ }^{1} \mathrm{H}-\mathrm{MRS}$, determination of hepatic lipid composition is hampered. Here, we developed, validated and applied ${ }^{1} \mathrm{H}-\mathrm{MRS}$ methodology that enabled us to non-invasively quantify the fractions of hepatic SFA, MUFA and PUFA separately. We showed that DNL was positively related to the hepatic SFA fraction and negatively to the MUFA fraction in overweight/obese participants, suggesting that high rates of DNL modify fatty acid composition. We confirmed this association between DNL and hepatic SFA fraction by showing increased hepatic SFA fraction in GSD1a patients, a genetic model of increased DNL. Furthermore, we showed that the hepatic SFA fraction is elevated in NAFL and diabetes type II patients and related to hepatic insulin resistance. These results show that our ${ }^{1} \mathrm{H}-\mathrm{MRS}$ protocol can be used to determine hepatic fat composition in humans and that particularly the SFA fraction can be seen as a non-invasive measure of DNL, directly measured in the liver. Furthermore, our results indicate that hepatic lipid composition may be a clinically important feature of hepatic fat accumulation.

Previously, a few studies have used similar approaches using ${ }^{1} \mathrm{H}-\mathrm{MRS}$ for hepatic lipid profiling, but none of these studies have reported SFA, MUFA and PUFA fractions separately (16-19). Even more so, in case of changes in MUFA/PUFA ratios, the calculation of the 
saturation index by using the methine resonance can lead to a misinterpretation of the lipid composition. We here show that by acquiring high quality liver spectra and by applying a sophisticated post-processing method for these spectra it is possible to differentiate between the hepatic SFA, MUFA and PUFA fractions in vivo in volunteers with and without NAFL. Implementation of this methodology in future studies makes it possible to further explore the importance of hepatic fat composition in metabolic health and the factors that could modulate fatty acid composition.

High rates of DNL may change hepatic lipid composition. The end product of the lipogenic pathway is palmitate $(\mathrm{C} 16: 0)$, which in turn can undergo elongation to stearate $(\mathrm{C} 18: 0)$ and desaturation by Stearoyl-CoA desaturase-1 (SCD1) to the MUFAs palmitoleate (C16:1 n-7) and oleate (C18:1 n-9). Previously, it has been shown that changes in DNL as determined by stable isotope techniques correlate with changes in the fraction of palmitate in VLDL-TG (32). In line with this, DNL was positively correlated with the hepatic SFA fraction in the current study. The hepatic MUFA fraction was negatively correlated with DNL, whereas the hepatic PUFA fraction did not correlate with DNL. From these results, it follows that upon increased DNL, the production of SFA is not necessarily accompanied by subsequent desaturation to MUFAs in humans. This is in contrast to animal studies that indicate that desaturation is upregulated in parallel to upregulation of lipogenic enzymes (13). In line, Peter et al. showed that SCD1 mRNA expression and $\mathrm{C} 18: 1 / \mathrm{C} 18: 0$ ratio in $\mathrm{TG}$ and phospholipid fractions in human liver were not increased in individuals with NAFL (33). Thus, handling of fatty acids originating from DNL by the liver may be different in humans and animals. Increased desaturation following lipogenesis is believed to be a rescue mechanism to reduce the negative effects exerted by saturated fatty acids (13). Further, we find higher hepatic SFA fraction in patients with NAFL and T2DM compared to controls, which is in line with earlier results, showing that the relative contribution of DNL derived FA to VLDL-TG was around $5-10 \%$ in healthy individuals in the fasted state, whereas their contribution increased to around $20-25 \%$ in people with NAFL $(11,30,34,35)$. These results suggest that the negative health effects that have been attributed to increased rates of DNL may also be due to the high fraction of SFA that result from DNL. Therefore, drug development targeting DNL is promising (36-38) although it is important to note that the evidence in the present study is only associative and future research will have to show whether decreasing the amount of SFA has beneficial effects on metabolic health. Interestingly however, animal studies support this notion. In mice overexpressing carbohydrate-responsive element-binding protein (ChREBP), resulting in 


\section{$\underline{\text { CHAPTER } 3}$}

increased activity of SCD1 and higher MUFA fraction, the increased MUFA fraction at the expense of SFA fraction has been associated with increased insulin sensitivity, despite high amounts of total hepatic fat content (13). Furthermore, it has been shown in vitro that specifically SFA negatively affect insulin signaling and that desaturation of fatty acids can rescue these negative effects (13). In line with these animal and cell data, we here show that the hepatic SFA fraction was negatively correlated and the MUFA/SFA ratio positively correlated with hepatic insulin sensitivity in overweight and obese participants. Plasma (VLDL-)TG is often used as a surrogate for hepatic TG. In addition, with sophisticated centrifugation methods, saturation of plasma VLDL-TG can be determined. Here we showed that saturation of hepatic lipids correlated with the saturation in plasma VLDL-TG. Interestingly however, unlike the MRS determined SFA fraction, the SFA fraction in VLDLTG did not correlate with DNL. These results indicate, that the SFA fraction determined by MRS is a better measure of DNL compared to the SFA fraction in VLDL-TG, and thus the use of our $1 \mathrm{H}-\mathrm{MRS}$ approach provides extra valuable information that could not be determined from plasma VLDL-TG.

\section{CONCLUSION}

We developed an MR protocol that enabled us to non-invasively quantify the fractions of hepatic SFA, MUFA and PUFA. Applying this ${ }^{1} \mathrm{H}-\mathrm{MRS}$ methodology, we here show that the hepatic SFA fraction is positively associated with DNL, is elevated in patients with NAFL and T2D, and negatively associated with hepatic insulin sensitivity. These results indicate that hepatic lipid composition, as determined by our ${ }^{1} \mathrm{H}-\mathrm{MRS}$ methodology, might be used as measure of DNL and furthermore suggest that specifically the hepatic SFA fraction may determine metabolic health.

\section{ACKNOWLEDGEMENTS}

This research was in part financed by the Ministry of Economic Affairs and Climate Policy by means of the PPP Allowance made available by the Top Sector Life Sciences \& Health to stimulate public-private partnerships and by Unilever R\&D Wageningen. We acknowledge the support from the Netherlands Cardiovascular Research Initiative: an initiative with support of the Dutch Heart Foundation (CVON2014-02 ENERGISE). LL is a recipient of a veni grant from ZonMW (016.veni.188.036) and a junior fellowship (2017.81.004) from the Diabetes Fonds (Dutch Diabetes Research Foundation). BS is a recipient of an Advanced ERC Grant (694717). VS is a recipient of an ERC starting grant (grant no. 759161 'MRS in diabetes'). 


\section{REFERENCES}

1. Sayiner M, Koenig A, Henry L, Younossi ZM. Epidemiology of Nonalcoholic Fatty Liver Disease and Nonalcoholic Steatohepatitis in the United States and the Rest of the World. Clin Liver Dis. 2016 May;20(2):205-14.

2. Gaggini M, Morelli M, Buzzigoli E, DeFronzo RA, Bugianesi E, Gastaldelli A. Non-Alcoholic Fatty Liver Disease (NAFLD) and Its Connection with Insulin Resistance, Dyslipidemia, Atherosclerosis and Coronary Heart Disease. Nutrients. 2013 May 10;5(5):1544-60.

3. Calzadilla Bertot L, Adams LA. The Natural Course of Non-Alcoholic Fatty Liver Disease. Int J Mol Sci. 2016 May 20;17(5).

4. Mantovani A, Byrne CD, Bonora E, Targher G. Nonalcoholic Fatty Liver Disease and Risk of Incident Type 2 Diabetes: A Meta-analysis. Diabetes Care. 2018 Feb;41(2):372-82.

5. Targher G, Byrne CD, Lonardo A, Zoppini G, Barbui C. Non-alcoholic fatty liver disease and risk of incident cardiovascular disease: A meta-analysis. J Hepatol. 2016 Sep;65(3):589-600.

6. Lim S, Oh TJ, Koh KK. Mechanistic link between nonalcoholic fatty liver disease and cardiometabolic disorders. Int J Cardiol. 2015 Dec 15;201:408-14.

7. Targher G, Byrne CD. Clinical Review: Nonalcoholic fatty liver disease: a novel cardiometabolic risk factor for type 2 diabetes and its complications. J Clin Endocrinol Metab. 2013 Feb;98(2):483-95.

8. Targher G, Day CP, Bonora E. Risk of cardiovascular disease in patients with nonalcoholic fatty liver disease. N Engl J Med. 2010 Sep 30;363(14):1341-50.

9. Brouwers B, Schrauwen-Hinderling VB, Jelenik T, Gemmink A, Havekes B, Bruls Y, et al. Metabolic disturbances of non-alcoholic fatty liver resemble the alterations typical for type 2 diabetes. Clin Sci Lond Engl 1979. 2017 Aug 1;131(15):1905-17.

10. Matikainen N, Adiels M, Söderlund S, Stennabb S, Ahola T, Hakkarainen A, et al. Hepatic lipogenesis and a marker of hepatic lipid oxidation, predict postprandial responses of triglyceride-rich lipoproteins. Obes Silver Spring Md. 2014 Aug;22(8):1854-9.

11. Lambert JE, Ramos-Roman MA, Browning JD, Parks EJ. Increased de novo lipogenesis is a distinct characteristic of individuals with nonalcoholic fatty liver disease. Gastroenterology. 2014 Mar; 146(3):72635 .

12. Gluchowski NL, Gabriel KR, Chitraju C, Bronson RT, Mejhert N, Boland S, et al. Hepatocyte Deletion of Triglyceride-Synthesis Enzyme Acyl CoA: Diacylglycerol Acyltransferase 2 Reduces Steatosis Without Increasing Inflammation or Fibrosis in Mice. Hepatol Baltim Md. 2019 Dec;70(6):1972-85.

13. Benhamed F, Denechaud P-D, Lemoine M, Robichon C, Moldes M, Bertrand-Michel J, et al. The lipogenic transcription factor ChREBP dissociates hepatic steatosis from insulin resistance in mice and humans. J Clin Invest. 2012 Jun;122(6):2176-94.

14. Puri P, Baillie RA, Wiest MM, Mirshahi F, Choudhury J, Cheung O, et al. A lipidomic analysis of nonalcoholic fatty liver disease. Hepatol Baltim Md. 2007 Oct;46(4):1081-90.

15. Araya J, Rodrigo R, Videla LA, Thielemann L, Orellana M, Pettinelli P, et al. Increase in long-chain polyunsaturated fatty acid $n-6 / n-3$ ratio in relation to hepatic steatosis in patients with non-alcoholic fatty liver disease. Clin Sci Lond Engl 1979. 2004 Jun;106(6):635-43.

16. Johnson NA, Walton DW, Sachinwalla T, Thompson CH, Smith K, Ruell PA, et al. Noninvasive assessment of hepatic lipid composition: Advancing understanding and management of fatty liver disorders. Hepatol Baltim Md. 2008 May;47(5):1513-23. 


\section{CHAPTER 3}

17. Hamilton G, Yokoo T, Bydder M, Cruite I, Schroeder ME, Sirlin CB, et al. In vivo characterization of the liver fat ${ }^{1} \mathrm{H}$ MR spectrum. NMR Biomed. 2011 Aug;24(7):784-90.

18. Lundbom J, Hakkarainen A, Söderlund S, Westerbacka J, Lundbom N, Taskinen M-R. Long-TE 1H MRS suggests that liver fat is more saturated than subcutaneous and visceral fat. NMR Biomed. 2011 Apr;24(3):238-45.

19. Gajdošík M, Chadzynski GL, Hangel G, Mlynárik V, Chmelík M, Valkovič L, et al. Ultrashort-TE stimulated echo acquisition mode (STEAM) improves the quantification of lipids and fatty acid chain unsaturation in the human liver at 7 T. NMR Biomed. 2015 Oct;28(10):1283-93.

20. Frahm J, Bruhn H, Gyngell ML, Merboldt KD, Hänicke W, Sauter R. Localized high-resolution proton NMR spectroscopy using stimulated echoes: initial applications to human brain in vivo. Magn Reson Med. 1989 Jan;9(1):79-93.

21. Tkác I, Starcuk Z, Choi IY, Gruetter R. In vivo 1H NMR spectroscopy of rat brain at $1 \mathrm{~ms}$ echo time. Magn Reson Med. 1999 Apr;41(4):649-56.

22. Dong Z, Dreher W, Leibfritz D. Experimental method to eliminate frequency modulation sidebands in localized in vivo $1 \mathrm{H}$ MR spectra acquired without water suppression. Magn Reson Med. 2004 Mar;51(3):602-6.

23. Diraison F, Pachiaudi C, Beylot M. In vivo measurement of plasma cholesterol and fatty acid synthesis with deuterated water: determination of the average number of deuterium atoms incorporated. Metabolism. 1996 Jul;45(7):817-21.

24. Dempster P, Aitkens S. A new air displacement method for the determination of human body composition. Med Sci Sports Exerc. 1995 Dec;27(12):1692-7.

25. Siri WE. Body composition from fluid spaces and density: analysis of methods. 1961. Nutr Burbank Los Angel Cty Calif. 1993 Oct;9(5):480-91; discussion 480, 492.

26. Löfgren L, Ståhlman M, Forsberg G-B, Saarinen S, Nilsson R, Hansson GI. The BUME method: a novel automated chloroform-free 96-well total lipid extraction method for blood plasma. J Lipid Res. 2012 Aug;53(8):1690-700.

27. Löfgren L, Forsberg G-B, Ståhlman M. The BUME method: a new rapid and simple chloroform-free method for total lipid extraction of animal tissue. Sci Rep. 2016 Jun 10;6(1):27688.

28. Murphy RC, James PF, McAnoy AM, Krank J, Duchoslav E, Barkley RM. Detection of the Abundance of Diacylglycerol and Triacylglycerol Molecular Species in Cells Using Neutral Loss Mass Spectrometry. Anal Biochem. 2007 Jul 1;366(1):59-70.

29. Steele R. Influences of glucose loading and of injected insulin on hepatic glucose output. Ann N Y Acad Sci. 1959 Sep 25;82:420-30.

30. Donnelly KL, Smith CI, Schwarzenberg SJ, Jessurun J, Boldt MD, Parks EJ. Sources of fatty acids stored in liver and secreted via lipoproteins in patients with nonalcoholic fatty liver disease. J Clin Invest. 2005 May;115(5):1343-51.

31. Bandsma RHJ, Prinsen BH, van Der Velden M de S, Rake J-P, Boer T, Smit GPA, et al. Increased de novo lipogenesis and delayed conversion of large VLDL into intermediate density lipoprotein particles contribute to hyperlipidemia in glycogen storage disease type 1a. Pediatr Res. 2008 Jun;63(6):702-7.

32. Hudgins LC, Parker TS, Levine DM, Hellerstein MK. A dual sugar challenge test for lipogenic sensitivity to dietary fructose. J Clin Endocrinol Metab. 2011 Mar;96(3):861-8. 
33. Peter A, Cegan A, Wagner S, Elcnerova M, Königsrainer A, Königsrainer I, et al. Relationships between hepatic stearoyl-CoA desaturase-1 activity and mRNA expression with liver fat content in humans. Am J Physiol-Endocrinol Metab. 2010 Nov 2;300(2):E321-6.

34. Timlin MT, Barrows BR, Parks EJ. Increased dietary substrate delivery alters hepatic fatty acid recycling in healthy men. Diabetes. 2005 Sep;54(9):2694-701.

35. Hodson L. Hepatic fatty acid synthesis and partitioning: the effect of metabolic and nutritional state. Proc Nutr Soc. 2019 Feb;78(1):126-34.

36. Kim C-W, Addy C, Kusunoki J, Anderson NN, Deja S, Fu X, et al. Acetyl CoA Carboxylase Inhibition Reduces Hepatic Steatosis but Elevates Plasma Triglycerides in Mice and Humans: A Bedside to Bench Investigation. Cell Metab. 2017 Aug 1;26(2):394-406.e6.

37. Loomba R, Kayali Z, Noureddin M, Ruane P, Lawitz EJ, Bennett M, et al. GS-0976 Reduces Hepatic Steatosis and Fibrosis Markers in Patients With Nonalcoholic Fatty Liver Disease. Gastroenterology. 2018 Nov;155(5):1463-1473.e6.

38. Lawitz EJ, Coste A, Poordad F, Alkhouri N, Loo N, McColgan BJ, et al. Acetyl-CoA Carboxylase Inhibitor GS-0976 for 12 Weeks Reduces Hepatic De Novo Lipogenesis and Steatosis in Patients With Nonalcoholic Steatohepatitis. Clin Gastroenterol Hepatol Off Clin Pract J Am Gastroenterol Assoc. 2018 Dec;16(12):1983-1991.e3. 


\section{SUPPLEMENTARY DATA}

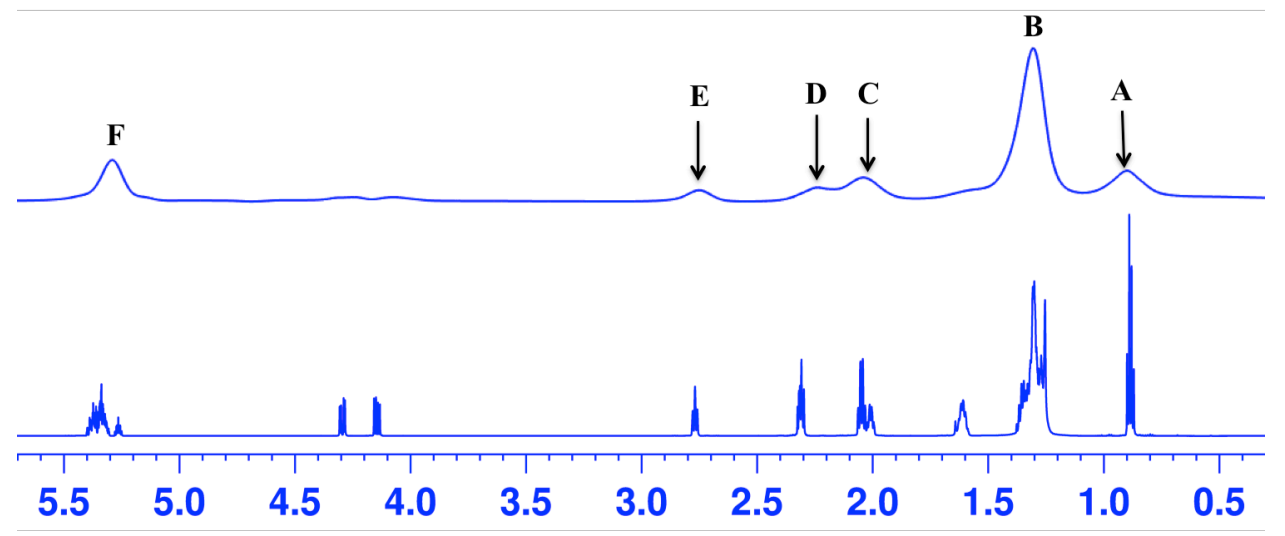

Supplementary figure 1: Comparison of lipid spectrum acquired at $3 T$ and high-resolution ${ }^{1} H-N M R$. Lipid spectrum acquired from the sunflower oil at $3 \mathrm{~T}$ (top) and high-resolution ${ }^{1} \mathrm{H}-\mathrm{NMR}$ spectrum (bottom) showing the variation in spectra resolution. A-methyl $\left(-\mathrm{CH}_{3}\right)$; B-methylene $\left(-\mathrm{CH}_{2}\right)$; C-allylic $\left(\mathrm{CH}_{3}-\mathrm{CH}=\mathrm{CH}-\mathrm{CH}_{2}\right) ; \quad$ D-alpha carbonyl $\left(\mathrm{CH}_{2}-\mathrm{COO}\right) ; \quad$ E-diallylic $\quad\left(=\mathrm{CH}-\mathrm{CH}_{2}-\mathrm{CH}=\right) ; \quad$ F-methine $(\mathrm{CH}=\mathrm{CH})$.

Supplementary table 1: Correction factors for PUFA and MUFA calculation.

\begin{tabular}{l|c|c}
\hline & CA & CB \\
\hline Olive oil & 0,759 & 0,997 \\
Arachis oil & 0,735 & 0,987 \\
Sunflower oil & 0,907 & 1,003 \\
Safflower oil & 0,889 & 0,982 \\
Rice oil & 0,849 & 0,990 \\
\hline
\end{tabular}


Supplementary table 2: List of triglyceride species determined by mass spectrometry.

\begin{tabular}{l} 
Triglyceride species \\
\hline TAG 48:0 \\
TAG 48:1 \\
TAG 48:2 \\
TAG 48:3 \\
TAG 50:0 \\
TAG 50:1 \\
TAG 50:2 \\
TAG 50:3 \\
TAG 50:4 \\
TAG 50:5 \\
TAG 52:0 \\
TAG 52:1 \\
TAG 52:2 \\
TAG 52:3 \\
TAG 52:4 \\
TAG 52:5 \\
TAG 52:6 \\
TAG 54:1 \\
TAG 54:2 \\
TAG 54:3 \\
TAG 54:4 \\
TAG 54:5 \\
TAG 54:6 \\
TAG 54:7 \\
TAG 56:2 \\
TAG 56:3 \\
TAG 56:4 \\
TAG 56:5 \\
TAG 56:6 \\
TAG 56:7 \\
TAG 56:8 \\
TAG 58:7 \\
TAG 58:8 \\
TAG 58:9 \\
\hline
\end{tabular}


Supplementary table 3: List of fatty acid species determined by mass spectrometry.

\begin{tabular}{l|l}
\hline FA species & Saturation \\
\hline FA 14:0 & SFA \\
FA 16:0 & SFA \\
FA 18:0 & SFA \\
FA 16:1 & MUFA \\
FA 18:1 & MUFA \\
FA 18:2 & PUFA \\
FA 20:3 & PUFA \\
FA 20:4 & PUFA \\
FA 20:5 & PUFA \\
FA 22:5 & PUFA \\
FA 22:6 & PUFA \\
\hline
\end{tabular}

Supplementary table 4: Clamp parameters in control participants, patients with NAFL and T2D.

\begin{tabular}{l|c|c|c}
\hline & Control $(\mathrm{n}=7)$ & NAFL $(\mathrm{n}=14)$ & T2D $(\mathrm{n}=9)$ \\
\hline $\mathrm{Ra}(\mu \mathrm{mol} / \mathrm{kg} / \mathrm{min})$ & & & \\
Baseline & $9.74 \pm 2.40$ & $7.64 \pm 1.50$ & $12.84 \pm 2.03^{\mathrm{A}, \mathrm{B}}$ \\
Low insulin & $11.88 \pm 1.34$ & $9.08 \pm 1.69^{\mathrm{A}}$ & $9.83 \pm 2.44$ \\
High insulin & $35.16 \pm 11.29$ & $26.58 \pm 7.74$ & $23.02 \pm 10.76$ \\
Rd ( $\mu$ mol/kg/min) & & & \\
Baseline & $8.87 \pm 2.06$ & $7.18 \pm 1.64$ & $13.28 \pm 3.10^{\mathrm{A}, \mathrm{B}}$ \\
Low insulin & $12.68 \pm 1.30$ & $9.04 \pm 1.58^{\mathrm{A}}$ & $10.28 \pm 2.47^{\mathrm{A}}$ \\
High insulin & $35.41 \pm 10.80$ & $26.16 \pm 7.51$ & $23.55 \pm 9.93^{\mathrm{A}}$ \\
EGP $(\mu \mathrm{mol} / \mathrm{kg} / \mathrm{min})$ & & & \\
Baseline & $9.74 \pm 2.40$ & $7.64 \pm 1.50$ & $12.84 \pm 2.03^{\mathrm{A}, \mathrm{B}}$ \\
Low insulin & $2.68 \pm 2.55$ & $3.15 \pm 1.22$ & $7.54 \pm 2.09^{\mathrm{A}, \mathrm{B}}$ \\
High insulin & $0.98 \pm 1.47$ & $1.07 \pm 0.81$ & $2.73 \pm 0.51^{\mathrm{A}, \mathrm{B}}$ \\
\% suppression low insulin & $74.20 \pm 18.40$ & $56.92 \pm 18.50$ & $41.61 \pm 12.15^{\mathrm{A}}$ \\
\% suppression high insulin & $89.33 \pm 15.82$ & $85.24 \pm 12.06$ & $78.44 \pm 4.03$ \\
\hline
\end{tabular}

Data are presented as mean \pm SD. Overweight/obese controls without NAFL (controls, $n=7$ ), overweight/obese with NAFL (NAFL, $n=14$ ), patients with type 2 diabetes (T2D, $n=9$ ). Ra baseline was significantly higher in T2D compared to control $(\mathrm{p}=0.010)$ and NAFL $\left(\mathrm{p}=2.0 \times 10^{-6}\right)$. Ra low insulin was significantly lower in NAFL compared to control $(\mathrm{p}=0.010)$. Rd baseline was significantly higher in T2D compared to control $(\mathrm{p}=0.002)$ and NAFL $\left(\mathrm{p}=3.0 \times 10^{-6}\right)$. Rd low insulin was significantly higher in control compared to NAFL $(\mathrm{p}=0.001)$ and T2D $(\mathrm{p}=0.045)$. Rd high insulin was significantly lower in T2D compared to control $(\mathrm{p}=0.045)$. EGP baseline was significantly higher in T2D compared to control $(\mathrm{p}=0.010)$ and NAFL $\left(\mathrm{p}=2.0 \times 10^{-6}\right)$. EGP low insulin was significantly higher in T2D compared to control $\left(\mathrm{p}=2.35 \times 10^{-4}\right)$ and NAFL $(\mathrm{p}=0.001)$. EGP high insulin was significantly higher in T2D compared to control $(\mathrm{p}=0.003)$ and NAFL $(\mathrm{p}=0.001)$. EGP suppression low insulin was significantly lower in T2D compared to control $(\mathrm{p}=0.002) .{ }^{\mathrm{A}}$ Significantly different from control, ${ }^{\mathrm{B}}$ significantly different from NAFL (Kruskal-Wallis $\mathrm{p}<0.05$ for EGP Low Insulin and EGP suppression high insulin, and one-way ANOVA $\mathrm{p}<0.05$ for all other parameters). Bonferroni correction was used for post-hoc analyses. 


\section{CHAPTER 4}

\section{Application of a BIlinear Rotation Decoupling (BIRD) filter in combination with $\mathbf{J}$-difference editing for indirect ${ }^{13} \mathrm{C}$ measurements in the human liver}

Pandichelvam Veeraiah, Kim Brouwers, Joachim E. Wildberger, Vera B. Schrauwen Hinderling and Lucas Lindeboom

Magnetic Resonance in Medicine. 2020 Dec;84(6):2911-2917. 


\section{$\underline{\text { CHAPTER } 4}$}

\section{ABSTRACT}

Background and aim: Recently, we introduced a quantum coherence-based method (geHSQC) for indirect ${ }^{13} \mathrm{C}$-MRS in the liver to track ${ }^{13} \mathrm{C}$-labeled lipids into the hepatic lipid pool in vivo. This approach is more robust in case of respiratory motion, however, inherently leads to a signal loss of $50 \%$ when compared to a conventional J-difference editing technique (JDE). Here, we intend to improve the robustness of a regular JDE (STEAM-ACED) with the use of BIlinear Rotation Decoupling filter (BIRD) filter to achieve 100\% higher signal gain when compared to ge-HSQC.

Materials and methods: To determine the efficiency of the BIRD filter ${ }^{1} \mathrm{H}-\left[{ }^{13} \mathrm{C}\right]$ lipid spectra were acquired on 3T from a peanut oil phantom, with three different MR sequences: ge-HSQC, STEAM-ACED and BIRD filter together with STEAM-ACED (BIRD-STEAM-ACED). Finally, our proposed method is tested in vivo in five healthy volunteers with varying liver fat content. In these volunteers we quantified the ${ }^{1} \mathrm{H}-\left[{ }^{13} \mathrm{C}\right]$-signal from the hepatic lipid pool and determined ${ }^{13} \mathrm{C}$ enrichment, which is expected to be $1.1 \%$ according to the natural abundance of ${ }^{13} \mathrm{C}$.

Results: The application of the proposed BIRD filter reduces the subtraction artifact of ${ }^{1} \mathrm{H}-$ $\left[{ }^{12} \mathrm{C}\right]$ lipid signal efficiently in JDE experiments, which leads to a signal gain of $100 \%$ of ${ }^{1} \mathrm{H}$ $\left[{ }^{13} \mathrm{C}\right]$-lipid signals when compared to the ge-HSQC. Phase distortions in vivo were minimal with the use of BIRD as compared to STEAM-ACED which enabled us to robustly quantify the ${ }^{13} \mathrm{C}$-enrichment in all five volunteers.

Conclusion: The BIRD-STEAM-ACED sequence is an efficient and promising tool for ${ }^{13} \mathrm{C}$ tracking experiments in the human liver in vivo.

Keywords: ${ }^{13} \mathrm{C}$ MRS, BIRD filter, ${ }^{13} \mathrm{C}$ tracking. 


\section{INTRODUCTION}

The application of ${ }^{13} \mathrm{C}$-MRS together with the administration of specific ${ }^{13} \mathrm{C}$ labeled substrate enables to measure a large number of metabolic fluxes noninvasively in vivo (1-3). When compared to direct ${ }^{13} \mathrm{C}$-MRS, the use of indirect ${ }^{13} \mathrm{C}$-MRS (i.e. the detection of ${ }^{1} \mathrm{H}$ signals that are coupled to a ${ }^{13} \mathrm{C}$-nuclei) leads to a large signal gain and allows localization of the MR signal with standard ${ }^{1} \mathrm{H}$-MRS localization techniques $(4,5)$. As the natural abundance of ${ }^{13} \mathrm{C}$ is only $1.1 \%$, the efficacy of the spectral editing sequences used is crucial for robust suppression of the large ${ }^{1} \mathrm{H}-\left[{ }^{12} \mathrm{C}\right]$ signals.

Editing of the ${ }^{1} \mathrm{H}-\left[{ }^{13} \mathrm{C}\right]$ signal (e.g. indirect ${ }^{13} \mathrm{C}$ ) is mainly performed in two different ways; either by using regular J-difference editing (JDE) methods or by making use of approaches that allow single-shot acquisitions by applying multiple quantum-coherence (MQC) based sequences (5). Recently, we demonstrated that it is possible to noninvasively follow the retention of labeled dietary ${ }^{13} \mathrm{C}$-lipids into the hepatic lipid pool in vivo using gradient enhanced heteronuclear single quantum coherence (ge-HSQC) (6). However, the drawback of applying ge-HSQC is that it inherently leads to a signal loss of $50 \%$ when compared to a conventional JDE sequence. Earlier studies have demonstrated the applicability of JDE techniques for ${ }^{13} \mathrm{C}$ tracking applications especially to study glucose metabolism in brain $(7,8)$. Such JDE techniques are quite challenging to apply directly in liver to track ${ }^{13} \mathrm{C}$ lipids, as respiratory motion will result in imperfect subtraction due to the (large) overlapping ${ }^{1} \mathrm{H}-\left[{ }^{12} \mathrm{C}\right]$ lipid signal at $1.3 \mathrm{ppm}$. In a previous study, a JDE sequence was applied on skeletal muscle and liver of rats to study lipid handling (9), however it has not been used in humans yet due to the problem of respiratory motion leading to subtraction artifacts. Therefore, the aim of this study was to increase the robustness of a regular JDE technique to increase the edited ${ }^{1} \mathrm{H}-\left[{ }^{13} \mathrm{C}\right]$ signals when compared to the ge-HSQC methodology, which is required to be able to track the low incorporation rates of ${ }^{13} \mathrm{C}$ lipids in the human hepatic fat pool in vivo.

A STEAM-ACED sequence (STEAM localization with Adiabatic Carbon Editing and Decoupling) has been proposed because of its short TE to minimize the effect of ${ }^{1} \mathrm{H}-{ }^{1} \mathrm{H}$ coupling on the acquired signal intensity (10). A BIRD filter (11) is basically a heteronuclear spin echo with delays equal to $1 / 2 \mathrm{JCH}$ to align ${ }^{1} \mathrm{H}$ spins coupled to ${ }^{12} \mathrm{C}$ and ${ }^{13} \mathrm{C}$ spins in the opposite phase (-y and y axes), which allows a $90^{\circ}$ pulse to align these vectors parallel and antiparallel to the main magnetic field ( $\mathrm{z}$ and $-\mathrm{z}$ axes) respectively. After a $90^{\circ}$ pulse, ${ }^{1} \mathrm{H}$ attached to ${ }^{12} \mathrm{C}$ spins are inverted and will recover based on $\mathrm{T}_{1}$ relaxation, while ${ }^{1} \mathrm{H}$ attached to 


\section{$\underline{\text { CHAPTER } 4}$}

${ }^{13} \mathrm{C}$ spins are along the positive z-axis. By choosing an optimal TI, it is possible to suppress the ${ }^{1} \mathrm{H}-\left[{ }^{12} \mathrm{C}\right]$ signal. Here, we hypothesize that pre-suppression of the strong ${ }^{1} \mathrm{H}-\left[{ }^{12} \mathrm{C}\right]$ lipid signal, by introducing a Bllinear Rotation Decoupling (BIRD) filter prior to a STEAM-ACED sequence, optimized for detection of ${ }^{1} \mathrm{H}-\left[{ }^{13} \mathrm{C}\right]$-lipid signals, will lead to an accurate and robust detection of the ${ }^{1} \mathrm{H}-\left[{ }^{13} \mathrm{C}\right]$ lipid signal irrespective of respiratory motion, with higher signal yield as compared to ge-HSQC.

\section{MATERIALS AND METHODS}

\section{General}

All MR experiments were performed on a 3T MR system (Achieva 3T-X Philips Healthcare, Best, Netherlands) using a double tuned ${ }^{1} \mathrm{H} /{ }^{13} \mathrm{C}$ surface coil (Rapid Biomedical $\mathrm{GmbH}$ ). The coil was specifically designed for indirect ${ }^{13} \mathrm{C}$ experiments in the liver, with two ${ }^{13} \mathrm{C}$ elements in quadrature arrangement (size of the elements: $13.2 \times 21 \mathrm{~cm}$, covering $13.2 \times 23.9 \mathrm{~cm}$ ) and two slightly larger ${ }^{1} \mathrm{H}$ elements in quadrature arrangement $(18 \times 20 \mathrm{~cm}$ and $15 \times 15 \mathrm{~cm}$, covering $18 \times 20 \mathrm{~cm}$ ) to achieve optimal sensitivity (expected at a depth of at least $8-12 \mathrm{~cm}$ ). All in vivo experiments were performed according to the protocol approved by the Institutional Human Medical Ethics Committee of Maastricht University Medical Centre (MUMC), Maastricht, Netherlands. A written informed consent was obtained from all the volunteers prior to participating in this study.

\section{MR sequences}

For ${ }^{1} \mathrm{H}-\left[{ }^{13} \mathrm{C}\right]$ editing of the lipid signals, a STEAM-ACED (10) sequence was used with a TE of $7.9 \mathrm{~ms}(\mathrm{TE} 1=\mathrm{TE} 2=3.95 \mathrm{~ms}=1 / 2 \mathrm{JCH})$. Spredrex pulses (Standard Philips $90^{\circ}$ excitation pulse) with duration of $2.75 \mathrm{~ms}$ were used on the ${ }^{1} \mathrm{H}$ channel for both excitation and selective refocusing of ${ }^{1} \mathrm{H}$ signals. During the TM (Mixing Time) period, an adiabatic inversion pulse with a bandwidth of $1600 \mathrm{~Hz}$ was placed on the ${ }^{13} \mathrm{C}$ channel to selectively invert the ${ }^{1} \mathrm{H}-\left[{ }^{13} \mathrm{C}\right]$ spins. The ${ }^{13} \mathrm{C}$ inversion pulse was used with $\mathrm{OFF} / \mathrm{ON}$ condition on alternate scans. Subtraction of the acquired data with and without ${ }^{13} \mathrm{C}$ inversion pulse provided MR signals only from ${ }^{1} \mathrm{H}$ attached to ${ }^{13} \mathrm{C}$ nuclei (2 times), whereas the sum provided MR signals from all compounds containing ${ }^{1} \mathrm{H}$ but not attached to ${ }^{13} \mathrm{C}$ nuclei (shown in Supporting Information Figure S1).

In a next experiment, the BIlinear Rotation Decoupling (BIRD) (11) filter was placed prior to the STEAM-ACED sequence. The inversion time (TI) was optimized in in vivo to achieve presuppression of the large hindering lipid- $\mathrm{CH}_{2}$ signal $\left({ }^{1} \mathrm{H}-\left[{ }^{12} \mathrm{C}\right]\right.$ spins $)$ at $1.3 \mathrm{ppm}$ in order to 
minimize the subtraction artifact. Block pulses were used in the BIRD filter on the ${ }^{1} \mathrm{H}$ channel for both excitation and selective refocusing of ${ }^{1} \mathrm{H}$ signals. A MLEV inversion pulse was used on the ${ }^{13} \mathrm{C}$ channel as shown in Figure 1, to selectively invert the ${ }^{13} \mathrm{C}-\mathrm{CH}_{2}$ resonances (Figure 1). In ge-HSQC sequence, the editing was performed with a MQC pulse sequence placed during the TM period (6).

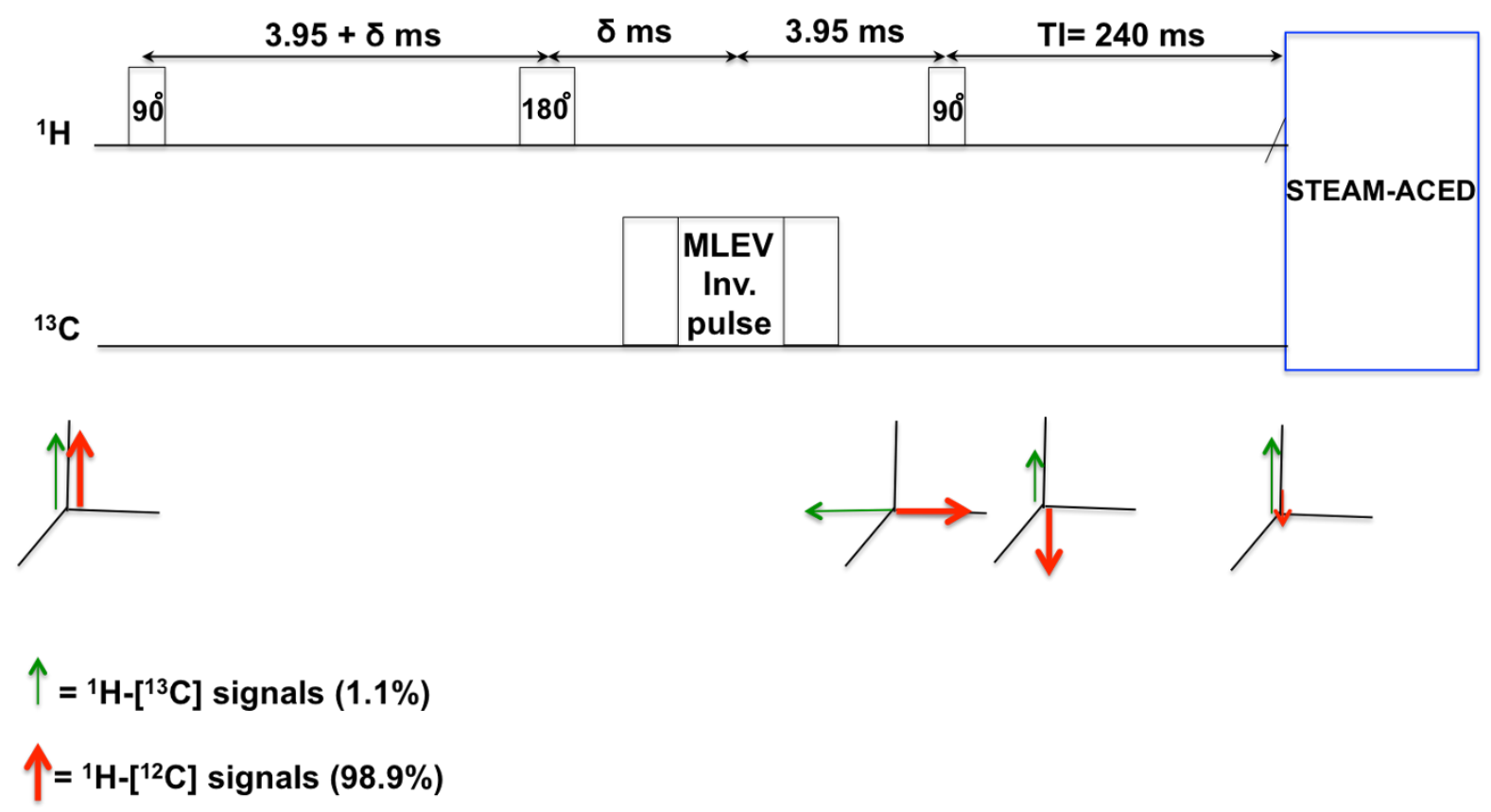

Figure 1: Application of a BIRD filter in front of a STEAM-ACED sequence. The filter is basically a spin echo sequence with a ${ }^{13} \mathrm{C}$ inversion pulse (MLEV) added to avoid J-refocusing of the ${ }^{1} \mathrm{H}-\left[{ }^{13} \mathrm{C}\right]$ signals, which will acquire opposite phase when compared to ${ }^{1} \mathrm{H}-\left[{ }^{12} \mathrm{C}\right]$ in the xy-plane by choosing the appropriate timings. The second $90^{\circ}$ pulse is used to flip the ${ }^{1} \mathrm{H}-\left[{ }^{12} \mathrm{C}\right]$ spins along the $-\mathrm{z}$-axis and the ${ }^{1} \mathrm{H}-\left[{ }^{13} \mathrm{C}\right]$ along the +z-axis. The inversion time (TI) is chosen according to the lipid $\mathrm{CH}_{2}-\mathrm{T}_{1}$ relaxation time in order to null the ${ }^{1} \mathrm{H}-\left[{ }^{12} \mathrm{C}\right]$ spins. As a result, only ${ }^{1} \mathrm{H}-\left[{ }^{13} \mathrm{C}\right]$ spins will be excited by the excitation pulse of the localization sequence (STEAM-ACED).

\section{Phantom experiments}

All MR sequence parameters and timings were optimized using a peanut oil phantom. Scout MR images were acquired with a turbo spin echo (TSE) sequence with TR/TE=2000/100 ms to place a voxel in the center of the phantom. Power calibration was performed using MEGAsLASER sequence to achieve maximal ${ }^{1} \mathrm{H}$ sensitivity in the volume of interest and the calculated power was kept same for both ${ }^{1} \mathrm{H}$ and ${ }^{13} \mathrm{C}$ channel throughout the experiment. Firstly, STEAM-ACED spectral editing sequence was used to acquire lipid ${ }^{1} \mathrm{H}-\left[{ }^{13} \mathrm{C}\right] \mathrm{MR}$ spectra, once with and without ${ }^{13} \mathrm{C}$ inversion with following parameters $\mathrm{TR} / \mathrm{TE}=4000 / 7.9 \mathrm{~ms}$, $\mathrm{NSA}=128$, voxel $=30 \times 30 \times 30 \mathrm{~mm}$, data points $=2048$. In a second experiment, the BIRD filter 
was used together with STEAM-ACED to acquire lipid spectra using identical parameters as above, but now with a TI of $240 \mathrm{~ms}$ in which the ${ }^{1} \mathrm{H}-\left[{ }^{12} \mathrm{C}\right]$ lipid signal was maximally suppressed. In a third experiment, ge-HSQC was used as previously described (6), to acquire ${ }^{1} \mathrm{H}-\left[{ }^{13} \mathrm{C}\right]$ lipid spectra with a $\mathrm{t} 1$ of $4 \mathrm{~ms}$. This set of experiments was used to compare the signal intensity and SNR of ${ }^{1} \mathrm{H}-\left[{ }^{13} \mathrm{C}\right]$ lipid signal and therefore to determine the efficiency of the BIRD filter. SNR was determined by calculating the ratio of the ${ }^{13} \mathrm{C}$ mean peak height and the standard deviation of noise in the edited spectrum.

\section{In vivo MR experiments}

Five healthy male volunteers (mean age $34 \pm 13$ years; mean BMI $28.6 \pm 3.7 \mathrm{~kg} / \mathrm{m}^{2}$ ) were included in this study to determine the in vivo feasibility of our proposed approach. The volunteers were positioned in supine position. The ${ }^{1} \mathrm{H} /{ }^{13} \mathrm{C}$ surface coil was placed on the right side of the chest in subject to cover the liver region. A fast scout MR image (slice thickness=15 $\mathrm{mm}$, flip angle $=15^{\circ}, \mathrm{FOV}=450 \times 450 \times 115 \mathrm{~mm}$ ) was acquired in three orientations (axial, coronal and transversal) with four breath holds to verify the positioning of the RF coil in the middle of the liver. A voxel of $45 \times 45 \times 45 \mathrm{~mm}$ was positioned in the liver by avoiding edges of the liver, diaphragm but also the biliary and vascular structures. STEAM-ACED was used to acquire hepatic lipid ${ }^{1} \mathrm{H}-\left[{ }^{13} \mathrm{C}\right] \mathrm{MR}$ spectra, once with and without ${ }^{13} \mathrm{C}$ inversion with the same MR parameters as used in the phantom. With the same experimental setup, additionally a separate experiment was performed using a BIRD filter prior to the STEAM-ACED sequence (TI=240 ms) to selectively pre-suppress the ${ }^{1} \mathrm{H}-\left[{ }^{12} \mathrm{C}\right]$ lipid signal. The $\mathrm{TI}=240 \mathrm{~ms}$ was chosen based on the average $\mathrm{T}_{1}$ relaxation time of lipid- $\mathrm{CH}_{2}$ in the liver $(340 \mathrm{~ms})$, predicting that the signal would be nulled after TI of $240 \mathrm{~ms}$. The long TR (4000 ms) was chosen to allow the volunteers to breath in the rhythm of the measurement (MR acquisition at end of expiration) and to avoid saturation effects due to $\mathrm{T}_{1}$ relaxation.

\section{Spectral analysis}

All obtained MRS data were post-processed with a custom-built Matlab (version R2017b; The Mathworks Inc.) script. The developed script allowed us to select both series of spectra acquired with and without editing pulses at once for each subject and allowed for automatic phasing, eddy current correction and frequency alignment of all spectra individually. Moreover, the script automatically removed spectra with poor quality based on a correlation approach, in which all individual spectra were ranked based on the correlation with all other spectra. An equal number of bad quality spectra were removed in both series acquired with and 
without editing pulse. Finally, the ${ }^{1} \mathrm{H}-\left[{ }^{13} \mathrm{C}\right]$ lipid resonances obtained in the edited spectrum were fitted as two identical peaks based on iterative based algorithm developed within the Matlab script, using prior knowledge on the known frequency shift of $127 \mathrm{~Hz}\left({ }^{1} \mathrm{H}-\left[{ }^{13} \mathrm{C}\right]\right.$ coupling for lipid). The best fit for the respective target lipid resonances were selected automatically when there was low residual (difference between fit and actual obtained spectrum) signal.

\section{Calculation of ${ }^{13} \mathrm{C}$ natural abundance and hepatic fat $\%$}

The total ${ }^{1} \mathrm{H}$ lipid signal coupled to both ${ }^{12} \mathrm{C}$ and ${ }^{13} \mathrm{C}$ spins were determined from the fitted peak of lipid- $\mathrm{CH}_{2}$ resonance of data acquired only with a conventional STEAM sequence (without ${ }^{13} \mathrm{C}$ inversion pulse in STEAM-ACED). The area of the ${ }^{1} \mathrm{H}-\left[{ }^{13} \mathrm{C}\right]$ lipid signal $\left({ }^{13} \mathrm{C}\right.$ natural abundance) was calculated by fitting and adding the two obtained ${ }^{1} \mathrm{H}-\left[{ }^{13} \mathrm{C}\right]$ lipid- $\mathrm{CH}_{2}$ resonances in the edited spectrum acquired with and without ${ }^{13} \mathrm{C}$ inversion pulse of STEAMACED. The percentage of ${ }^{13} \mathrm{C}$ enrichment was determined using the equation as shown below. Similarly, the ${ }^{13} \mathrm{C}$ lipid signal was also calculated from the data acquired with BIRD-STEAMACED sequence. Then, we compared the calculated percentage of ${ }^{13} \mathrm{C}$ enrichment in both sequences to determine the percentage of signal loss from the expected value of $1.1 \%$ (natural abundance). The hepatic fat $\%$ was calculated after $\mathrm{T}_{2}$ correction (water $\mathrm{T}_{2}=25.1 \mathrm{~ms}$; lipid $\left.\mathrm{T}_{2}=56.7 \mathrm{~ms}\right)$, using the ratio of $\mathrm{CH}_{2} /\left(\mathrm{CH}_{2}+\mathrm{H}_{2} \mathrm{O}\right)$ from the two spectra obtained with and without water suppression, acquired at $\mathrm{TE}=20 \mathrm{~ms}$ using conventional STEAM sequence.

$$
C^{13} \% \text { enrichment }=\left\{\frac{0.5 * S\left[C^{13}\right]}{S[\text { total }]}\right\} * 100
$$

Where $\mathrm{S}_{\text {[total] }}$ represents the total ${ }^{1} \mathrm{H}$ lipid- $\mathrm{CH}_{2}$ signal coupled to both ${ }^{12} \mathrm{C}$ and ${ }^{13} \mathrm{C}$ spins (peak at $1.3 \mathrm{ppm}$ in STEAM spectra) and $\mathrm{S}\left[\mathrm{C}^{13}\right]$ indicates only the addition of two signal intensities of ${ }^{1} \mathrm{H}-\left[{ }^{13} \mathrm{C}\right]$ lipid resonances obtained in the edited spectrum.

\section{RESULTS}

\section{Phantom}

The lipid spectra acquired from the peanut oil phantom using the three different MR sequences are depicted in Figure 2. As expected, the subtraction artifact of ${ }^{1} \mathrm{H}-\left[{ }^{12} \mathrm{C}\right]$ lipid signal was largely reduced with the ge-HSQC sequence when compared to a regular JDE sequence (STEAM-ACED; Figure 2A and 2B). Moreover, as expected, the measured ${ }^{13} \mathrm{C} \%$ enrichment 
with STEAM-ACED was found to be $0.94 \%$ in an oil phantom, indicating only $15 \%$ signal loss, as the obtained value is close to the known value of $1.1 \%\left({ }^{13} \mathrm{C}\right.$ natural abundance).
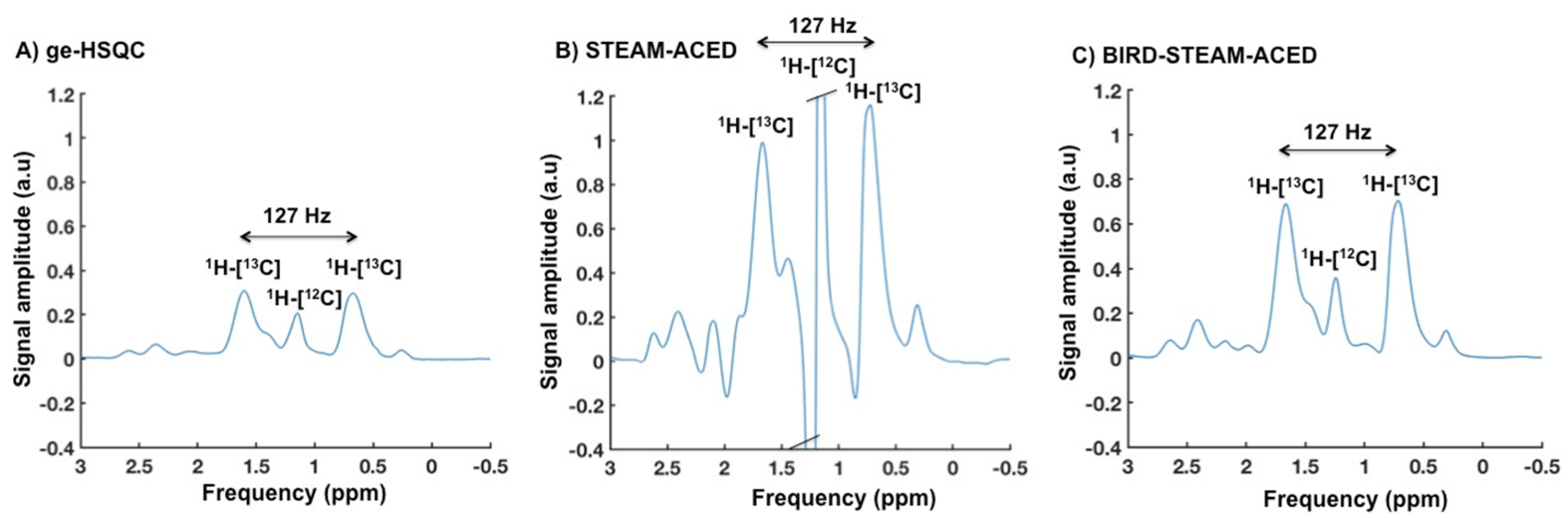

Figure 2: ${ }^{1} \mathrm{H}-I^{13} \mathrm{Cl}$ lipid spectra obtained from the peanut oil phantom with three different MR sequences. A) ge-HSQC B) STEAM-ACED C) BIRD filter together with STEAM-ACED.

Interestingly, the proposed sequence with the addition of BIRD filter reduces the subtraction artifact of the ${ }^{1} \mathrm{H}-\left[{ }^{12} \mathrm{C}\right]$ lipid signal in the oil phantom when compared to a conventional STEAM-ACED sequence. Moreover, the obtained ${ }^{1} \mathrm{H}-\left[{ }^{13} \mathrm{C}\right]$ lipid signal intensity with the use of BIRD filter was approximately two-fold higher SNR (173 vs 91) as compared to the previously proposed ge-HSQC method (Figure $2 \mathrm{C}$ ). The measured ${ }^{13} \mathrm{C}$ natural abundance with the BIRD-STEAM-ACED sequence in an oil phantom was found to be $0.74 \%$, indicating $21 \%$ signal loss in $1 \mathrm{H}-\left[{ }^{13} \mathrm{C}\right]$ lipid signal intensity due to BIRD filter as compared to the STEAMACED sequence alone or if SNR is considered, we find a 16\% loss in SNR (206 vs 173).

\section{In vivo}

Our new method was successfully applied in vivo and demonstrated the feasibility to detect ${ }^{13} \mathrm{C}$ natural abundance in volunteers with wide range of liver fat content ( 2 to $21 \%$, Table 1 ). As expected, a large subtraction artifact was apparent in in vivo when a conventional STEAMACED sequence was used (Figure 3A). This artifact leads to phase distortions in the edited spectrum, which hampers the accurate and robust quantification of the ${ }^{1} \mathrm{H}-\left[{ }^{13} \mathrm{C}\right]$ lipid signal (Figure 3A). The addition of the BIRD-filter leads to pre-suppression of the huge ${ }^{1} \mathrm{H}-\left[{ }^{12} \mathrm{C}\right]-$ lipid signal during MR acquisition and thereby leads to marked reduction in the subtraction artefact of the ${ }^{1} \mathrm{H}-\left[{ }^{12} \mathrm{C}\right]$ lipid signal (Figure $3 \mathrm{~B}$ ).

Due to the implementation of BIRD filter, we were able to visualize our target ${ }^{1} \mathrm{H}-\left[{ }^{13} \mathrm{C}\right]$ lipid signal clearly as two distinct peaks with known coupling constant of $127 \mathrm{~Hz}$. We were able to quantify the ${ }^{1} \mathrm{H}-\left[{ }^{13} \mathrm{C}\right]$ lipid signal successfully in all five volunteers. The SNR was sufficient, 
even in the subject with very low hepatic lipid content. Interestingly, the phase distortions in vivo were minimal with the use of the BIRD filter when compared to STEAM-ACED (Figure 3B). The measured ${ }^{1} \mathrm{H}-\left[{ }^{13} \mathrm{C}\right]$ lipid signal in all five volunteers with the use of BIRD-STEAMACED sequence are shown in the Table 1.

A)

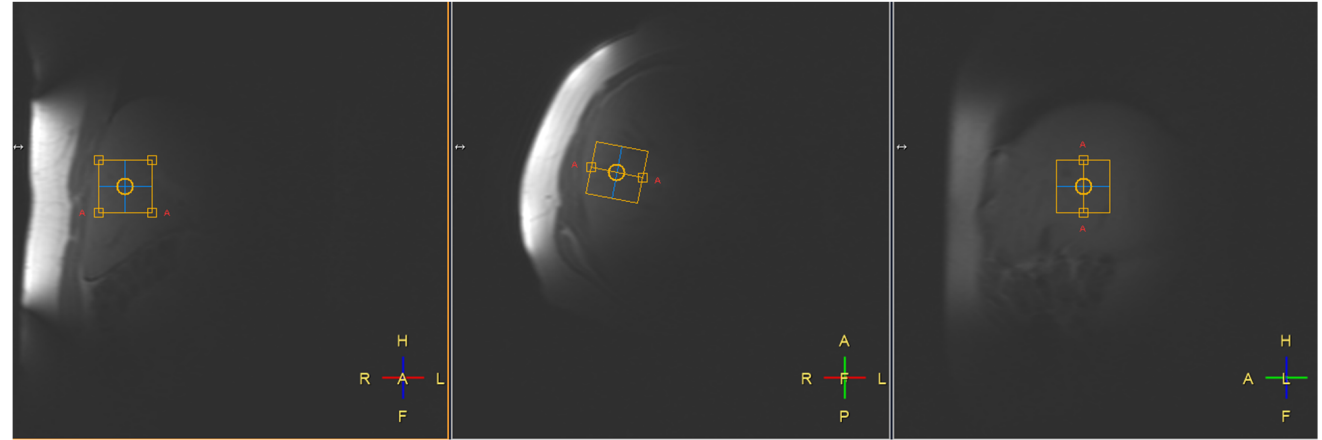

B) STEAM-ACED

C) BIRD-STEAM-ACED
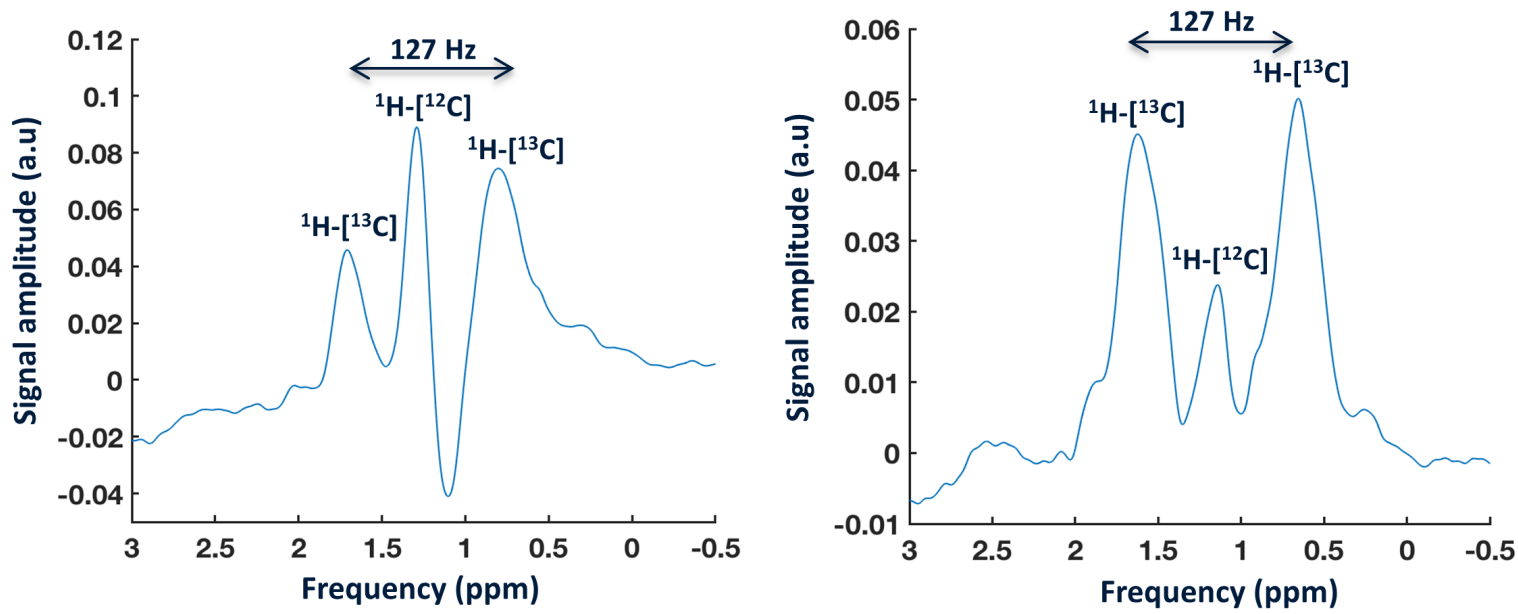

Figure 3: ${ }^{1} \mathrm{H}-I^{13} \mathrm{C}$ hepatic lipid spectra obtained from a healthy volunteer with and without BIRD filter. A) Screenshot of scout MR image showing the voxel position in three different orientations of a volunteer. ${ }^{1} \mathrm{H}-\left[{ }^{13} \mathrm{C}\right]$ hepatic lipid spectra obtained from the voxel with two different MR sequences $\mathrm{B}$ ) STEAM-ACED C) BIRD filter together with STEAM-ACED.

Table 1: The measured $\left.{ }^{1} \mathrm{H}-\Gamma^{13} \mathrm{C}\right]$ lipid signal (natural abundance) with BIRD-STEAM-ACED sequence in five different volunteers with varying hepatic fat content (\%).

\begin{tabular}{l|c|c}
\hline & Fat \% & ${ }^{\mathbf{1 3}} \mathbf{C} \%$-BIRD-STEAM-ACED \\
\hline Subject 1 & 13.8 & 0.58 \\
Subject 2 & 3.5 & 0.63 \\
Subject 3 & 3.3 & 0.54 \\
Subject 4 & 20.6 & 0.53 \\
Subject 5 & 2.4 & 0.63 \\
\hline \multicolumn{2}{c}{ Mean } & $0.58 \pm 0.05$ \\
\hline
\end{tabular}




\section{DISCUSSION}

In this study, we improved the robustness of a regular JDE (STEAM-ACED sequence) technique for the application in human liver with the use of BIRD filter and demonstrated the in vivo feasibility of our proposed approach. We were successful in the determination of the ${ }^{13} \mathrm{C}$ (natural abundance) lipids in hepatic fat pool in all five volunteers. The subtraction artifact of the ${ }^{1} \mathrm{H}-\left[{ }^{12} \mathrm{C}\right]$ lipid signal is efficiently reduced with the use of BIRD filter as compared to the conventional STEAM-ACED. Therefore, the two distinct peaks of the ${ }^{1} \mathrm{H}-\left[{ }^{13} \mathrm{C}\right]$ lipid- $\mathrm{CH}_{2}$ signal were clearly visible with the use of BIRD filter together with STEAM-ACED spectral editing sequence. Interestingly, with BIRD filter, there is a minimum signal loss in ${ }^{13} \mathrm{C}$ lipid signal intensity in phantom as compared to the conventional STEAM-ACED.

The addition of the BIRD filter leads to inherent ${ }^{1} \mathrm{H}-\left[{ }^{13} \mathrm{C}\right]$ signal loss, probably due to ${ }^{1} \mathrm{H}-{ }^{1} \mathrm{H}$ couplings. However, due to $\mathrm{T}_{1}$ recovery, the ${ }^{1} \mathrm{H}-\left[{ }^{13} \mathrm{C}\right]$ signal losses due to $\mathrm{BIRD}$ filter will be less with longer TI (when TI > actual null TI of lipid- $\mathrm{CH}_{2}$ ), which results in higher ${ }^{1} \mathrm{H}-\left[{ }^{13} \mathrm{C}\right]$ signal intensity. We aimed at nulling the ${ }^{1} \mathrm{H}-\left[{ }^{12} \mathrm{C}\right]$ signal with the chosen TI. A longer TI would provide higher ${ }^{1} \mathrm{H}-\left[{ }^{13} \mathrm{C}\right]$ lipid signal intensity, but simultaneously the ${ }^{1} \mathrm{H}-\left[{ }^{12} \mathrm{C}\right]$ residue will also be increased. Thus, there is a tradeoff between ${ }^{12} \mathrm{C}$ and ${ }^{13} \mathrm{C}$ lipid signals. Due to differences in $\mathrm{T}_{1}$ relaxation time between in vivo and the oil phantom (340 vs $270 \mathrm{~ms}$ ), the degree of recovery is slightly different and therefore, the oil phantom provides higher enrichment $(0.74 \%)$ than in vivo $(0.58 \%)$ while using identical TI (shown in Supporting Information Figure S2).

Moreover, both phantom and in vivo measurements yield lower ${ }^{13} \mathrm{C}$ enrichment than expected $\left({ }^{13} \mathrm{C}\right.$ natural abundance of $1.1 \%$ ). This indicates that there is (systemic) inherent signal loss, which might be due to ${ }^{1} \mathrm{H}-{ }^{1} \mathrm{H}$ couplings. However, this is not a problem for ${ }^{13} \mathrm{C}$ tracking applications in liver when indirect ${ }^{13} \mathrm{C}$ MRS is generally used to measure changes in the ${ }^{13} \mathrm{C} \%$ enrichment, as the baseline enrichment can be set to $1.1 \%$. This inherent signal loss will be taken into account to calculate the absolute ${ }^{13} \mathrm{C} \%$ enrichment.

Interestingly, the in vivo measured ${ }^{13} \mathrm{C}$ lipid signal enrichment (natural abundance) with $\mathrm{BIRD}$ STEAM-ACED sequence was very similar among all volunteers with varying liver fat content, suggesting that the present approach is very robust. Due to the insertion of the B1-insensitive MLEV pulse on the ${ }^{13} \mathrm{C}$ channel in the BIRD filter, signal loss is minimized, irrespective of $\mathrm{B} 1$ inhomogeneity while using a surface coil. In contrast, some B1 inhomogeneity is expected due to the use of standard pulses on the ${ }^{1} \mathrm{H}$ channel, and while this can lead to some signal loss in 
absolute terms, it will not affect the estimation of ${ }^{13} \mathrm{C}$ enrichment, as both the ${ }^{1} \mathrm{H}-\left[{ }^{13} \mathrm{C}\right]$ signal and the reference signal are equally affected. Due to phase distortions and a large residual of ${ }^{12} \mathrm{C}$ artifact, approaches to quantify the ${ }^{1} \mathrm{H}-\left[{ }^{13} \mathrm{C}\right]$ lipid signal intensity with solely STEAMACED were not successful in vivo. Thus, our proposed sequence with BIRD filter was designed to minimized ${ }^{1} \mathrm{H}-\left[{ }^{12} \mathrm{C}\right]$ signal and was successfully applied in in vivo in volunteers with varying total liver fat content and showed higher signal-to-noise ratio (SNR) for ${ }^{13} \mathrm{C}$ lipids (natural abundance) even in the subject with low liver fat content.

Also, the ge-HSQC provides excellent editing of the ${ }^{1} \mathrm{H}-\left[{ }^{13} \mathrm{C}\right]$ lipid- $\mathrm{CH}_{2}$ peak in an oil phantom but the signal loss of $>50 \%$ as compared to a conventional STEAM-ACED is unavoidable. Our proposed sequence with BIRD filter leads to a large signal gain in ${ }^{1} \mathrm{H}-\left[{ }^{13} \mathrm{C}\right]$-lipid signal intensity and SNR (almost two-fold) when compared to ge-HSQC. Next to this, the use of JDE in combination of BIRD also has other advantages when compared to ge-HSQC. Due to the relatively long $T_{1}$, the hepatic water signal intensity is not much affected by the optimized TI of $240 \mathrm{~ms}$. Therefore, the residual water signal can be used to perform phase and frequency alignment of individual spectra whereas in ge-HSQC this is not possible due to the gradient de-phasing of all ${ }^{1} \mathrm{H}-\left[{ }^{12} \mathrm{C}\right]$ lipid signals and also water signals (6). Also, a signal gain is expected when compared to ge-HSQC in practice due to the insertion of B1 insensitive pulses in our approach (MLEV pulse in BIRD filter and adiabatic hyperbolic secant pulse in the TM period of the STEAM-ACED sequence). In contrast to ge-HSQC, miscalibration of the ${ }^{13} \mathrm{C}$ transmit power does not per se lead to lower a ${ }^{13} \mathrm{C}$-enrichment, due to the $\mathrm{B} 1$ insensitivity of the pulses used in the proposed approach. The higher ${ }^{13} \mathrm{C}$ signal (as compared to ge-HSQC) together with minimal ${ }^{12} \mathrm{C}$ artifact with the use of BIRD filter will enable to track even the smaller contribution of labeled glucose (12) to form ${ }^{13} \mathrm{C}$ lipids (as compared to the contribution from ${ }^{13} \mathrm{C}$ labeled fatty acids) in hepatic fat pool. Thus, our proposed approach can now be used as an efficient and promising tool for ${ }^{13} \mathrm{C}$ tracking applications in vivo.

\section{CONCLUSION}

The proposed sequence with BIRD filter reduces the subtraction artifacts of ${ }^{1} \mathrm{H}-\left[{ }^{12} \mathrm{C}\right]$ lipid signal efficiently without much signal loss in the target ${ }^{1} \mathrm{H}-\left[{ }^{13} \mathrm{C}\right]$ lipid signal and thus, it can be used as an indirect ${ }^{13} \mathrm{C}$ method for ${ }^{13} \mathrm{C}$ tracking applications in liver. 


\section{$\underline{\text { CHAPTER } 4}$}

\section{ACKNOWLEDGEMENTS}

This research was in part financed by the Ministry of Economic Affairs and Climate Policy by means of the PPP Allowance made available by the Top Sector Life Sciences \& Health to stimulate public-private partnerships and by Unilever Foods Innovation Centre Wageningen. LL was a recipient of a veni grant from ZonMW (016.veni.188.036) and a junior fellowship (2017.81.004) from the Diabetes Fonds (Dutch Diabetes Research Foundation). VS-H was supported by an ERC starting grant (grant no. 759161 "MRS in diabetes"). 


\section{REFERENCES}

1. Rothman DL, De Feyter HM, de Graaf RA, Mason GF, Behar KL. 13C MRS studies of neuroenergetics and neurotransmitter cycling in humans. NMR Biomed. 2011 Oct;24(8):943-57.

2. Beckmann N, Turkalj I, Seelig J, Keller U. 13C NMR for the assessment of human brain glucose metabolism in vivo. Biochemistry. 1991 Jul 2;30(26):6362-6.

3. Petersen KF, Befroy DE, Dufour S, Rothman DL, Shulman GI. Assessment of Hepatic Mitochondrial Oxidation and Pyruvate Cycling in NAFLD by (13)C Magnetic Resonance Spectroscopy. Cell Metab. 2016 $12 ; 24(1): 167-71$.

4. Chen H, De Feyter HM, Brown PB, Rothman DL, Cai S, de Graaf RA. Comparison of direct 13C and indirect $1 \mathrm{H}-[13 \mathrm{C}] \mathrm{MR}$ detection methods for the study of dynamic metabolic turnover in the human brain. J Magn Reson San Diego Calif 1997. 2017;283:33-44.

5. de Graaf RA, Rothman DL, Behar KL. State of the art direct 13C and indirect 1H-[13C] NMR spectroscopy in vivo. A practical guide. NMR Biomed. 2011 Oct;24(8):958-72.

6. Lindeboom L, de Graaf RA, Nabuurs CI, van Ewijk PA, Hesselink MK, Wildberger JE, et al. Quantum coherence spectroscopy to measure dietary fat retention in the liver. JCI Insight. 2016 Aug 18;1(13):e84671.

7. de Graaf RA, Mason GF, Patel AB, Behar KL, Rothman DL. In vivo 1H-[13C]-NMR spectroscopy of cerebral metabolism. NMR Biomed. 2003 Nov;16(6-7):339-57.

8. Fitzpatrick SM, Hetherington HP, Behar KL, Shulman RG. The flux from glucose to glutamate in the rat brain in vivo as determined by $1 \mathrm{H}$-observed, 13C-edited NMR spectroscopy. J Cereb Blood Flow Metab Off J Int Soc Cereb Blood Flow Metab. 1990 Mar;10(2):170-9.

9. Jonkers RAM, Geraedts TR, van Loon LJC, Nicolay K, Prompers JJ. Multitissue assessment of in vivo postprandial intracellular lipid partitioning in rats using localized $1 \mathrm{H}-[13 \mathrm{C}]$ magnetic resonance spectroscopy. Magn Reson Med. 2012 Oct;68(4):997-1006.

10. Pfeuffer J, Tkác I, Choi IY, Merkle H, Ugurbil K, Garwood M, et al. Localized in vivo 1H NMR detection of neurotransmitter labeling in rat brain during infusion of [1-13C] D-glucose. Magn Reson Med. 1999 Jun;41(6):1077-83.

11. Garbow JR, Weitekamp DP, Pines A. Bilinear rotation decoupling of homonuclear scalar interactions. Chem Phys Lett. 1982 Dec 17;93(5):504-9.

12. Silva JCP, Marques C, Martins FO, Viegas I, Tavares L, Macedo MP, et al. Determining contributions of exogenous glucose and fructose to de novo fatty acid and glycerol synthesis in liver and adipose tissue. Metab Eng. 2019 Dec 1;56:69-76. 


\section{SUPPLEMENTARY DATA}

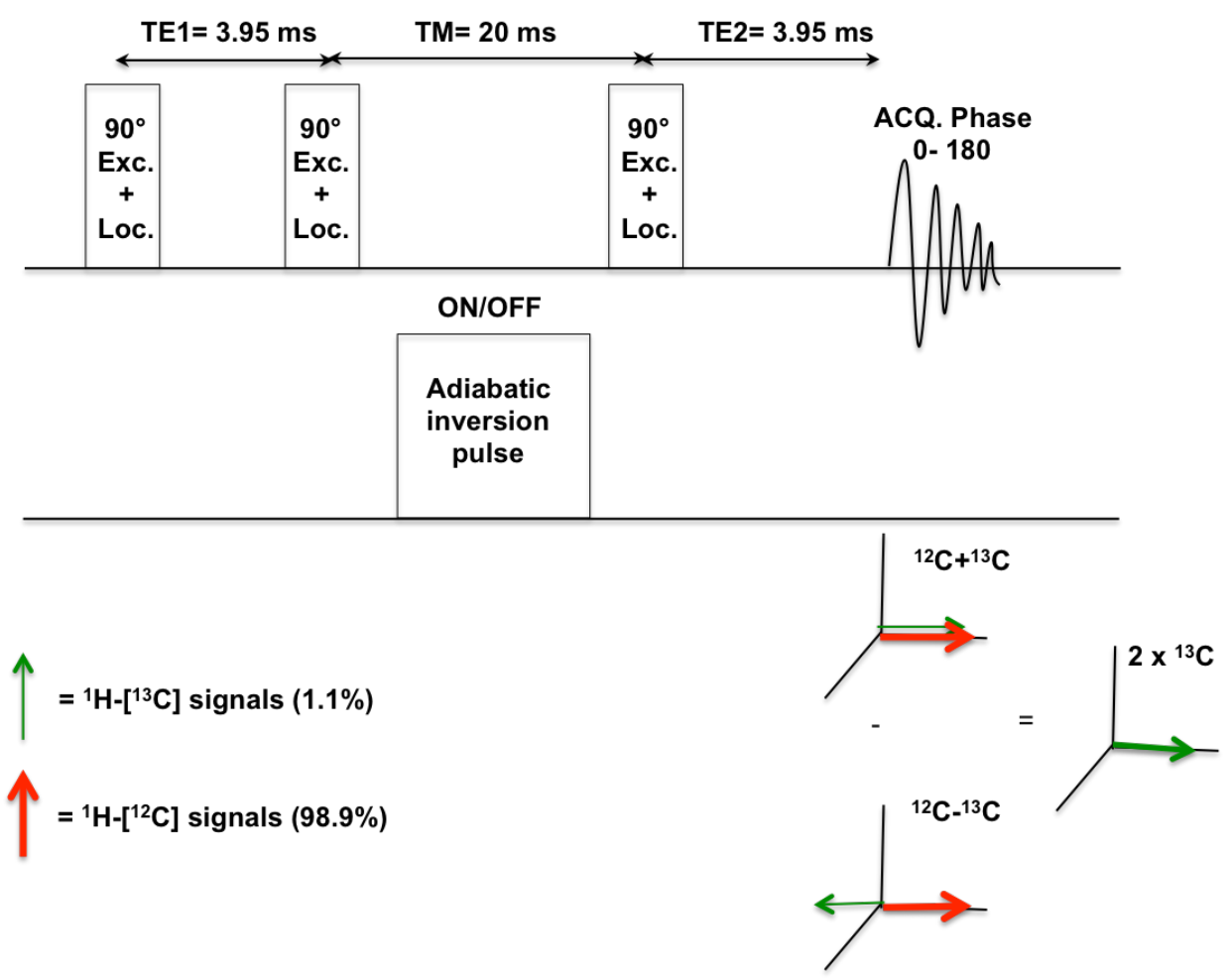

Supporting information figure S1: Sequence diagram of STEAM-ACED sequence(10). An adiabatic inversion pulse with $\mathrm{BW}$ of $1600 \mathrm{~Hz}$ is only applied on ${ }^{13} \mathrm{C}$ channel on alternate scans for editing purpose. 


\section{A) Peanut oil phantom}

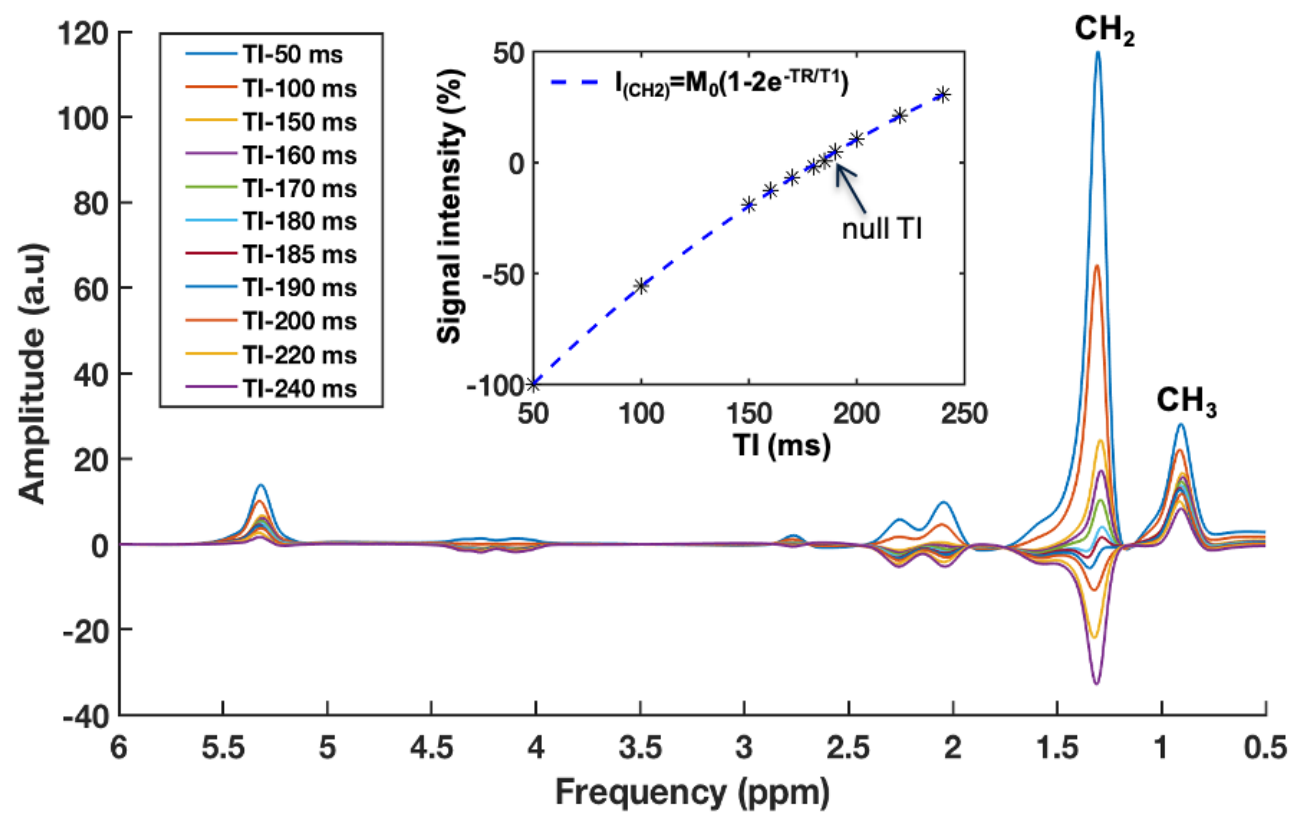

\section{B) Healthy volunteer}

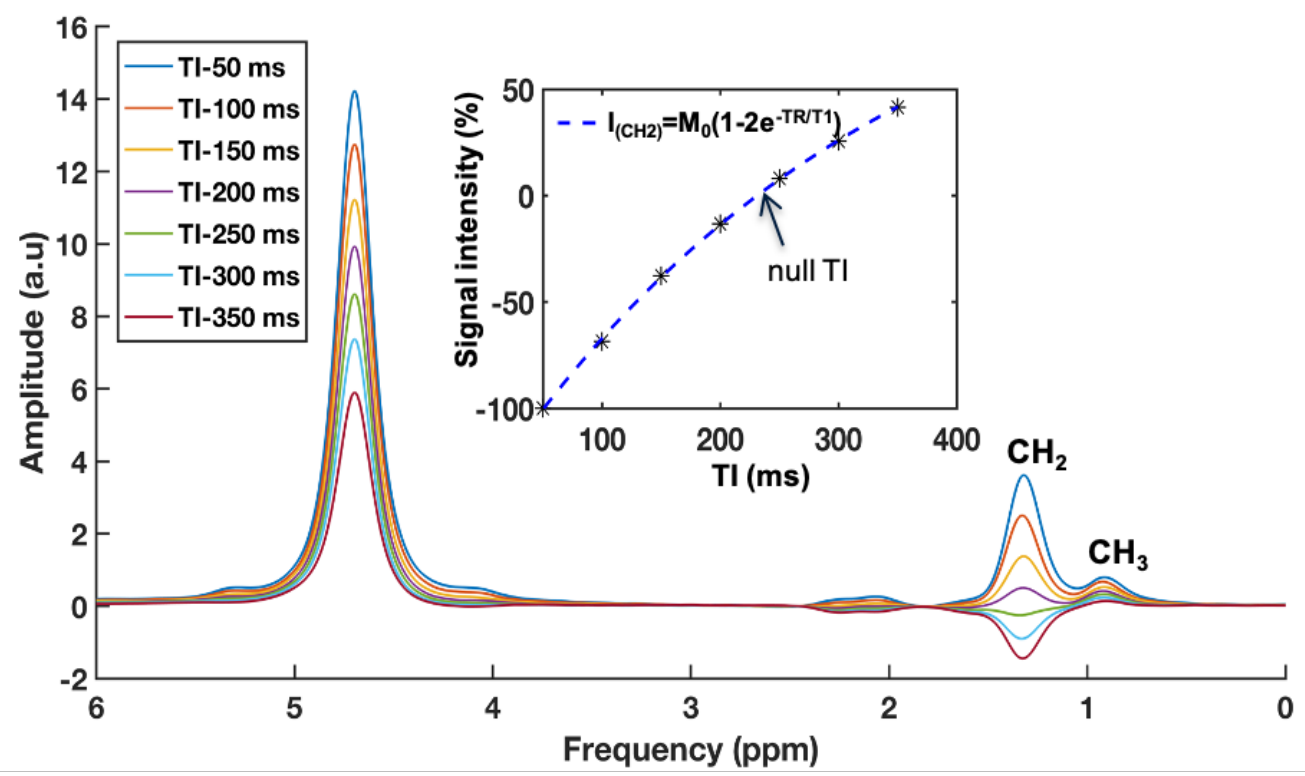

Supporting information figure S2: The difference of lipid $\mathrm{CH}_{2} \mathrm{~T}_{1}$ relaxation time between oil phantom and in vivo. ${ }^{1} \mathrm{H}-\mathrm{MR}$ lipid spectra acquired with different $\mathrm{TI}$ values from the peanut oil phantom (A) and from a healthy volunteer (B) using an inversion recovery with STEAM sequence. Plotted the signal intensity of lipid- $\mathrm{CH}_{2}$ versus TI in the respective figure. The null TI (indicated by arrow) for the lipid- $\mathrm{CH}_{2}$ was found to be $186 \mathrm{~ms}$ and $240 \mathrm{~ms}$ in oil phantom and in vivo respectively. Therefore, $\mathrm{T}_{1}$ of lipid- $\mathrm{CH}_{2}$ was estimated to be $270 \mathrm{~ms}$ in this oil phantom and $340 \mathrm{~ms}$ in in vivo. 



\section{CHAPTER 5}

\section{Effects of fructose restriction on liver steatosis (FRUITLESS); a double-blind randomized controlled trial}

Nynke Simons, Pandichelvam Veeraiah, Pomme I.H.G. Simons, Nicolaas C. Schaper, M. Eline Kooi, Vera B. Schrauwen-Hinderling, Edith J.M. Feskens, E.M.C. (Liesbeth) van der Ploeg, Mathias D.G. Van den Eynde, Casper G. Schalkwijk, Coen D.A. Stehouwer and Martijn C.G.J. Brouwers

The American Journal of Clinical Nutrition. 2021 Feb 2;113(2):391-400. 


\section{$\underline{\text { CHAPTER } 5}$}

\section{ABSTRACT}

Background and aim: There is an ongoing debate on whether fructose plays a role in the development of non-alcoholic fatty liver disease (NAFLD). The aim of this study was to investigate the effects of fructose restriction on intrahepatic lipid (IHL) content in a doubleblind randomized controlled trial using an isocaloric comparator.

Materials and methods: Between March 2017 and October 2019, forty-four adult overweight individuals with a fatty liver index $\geq 60$ were randomly assigned to a six-week fructoserestricted diet ( $<7.5$ grams per meal and $<10$ grams per day) supplemented with sachets of glucose (=intervention group) or fructose (=control group) three times daily. Participants and assessors were blinded to the allocation. IHL content, assessed by proton magnetic resonance spectroscopy, was the primary outcome and glucose tolerance and serum lipids were the secondary outcomes. All measurements were conducted in Maastricht University Medical Center.

Results: Thirty-seven participants completed the study protocol. After six weeks of fructose restriction, dietary fructose intake and urinary fructose excretion were significantly lower in the intervention group (difference: -57.0 grams/day, 95\%CI:-77.9, -39.5 and $-38.8 \mu \mathrm{mol} / \mathrm{day}$, 95\%CI:-91.2, -10.7, respectively). Although IHL content decreased in both the intervention and control group ( $\mathrm{p}<0.001$ and $\mathrm{p}=0.003$, respectively), the change in IHL content was more pronounced in the intervention group (difference: -0.7 percent point, $95 \% \mathrm{CI}:-2.0,-0.03$ ). The change in glucose tolerance and serum lipids were not significantly different between both groups.

Conclusions: Six weeks of fructose restriction per se leads to a small, but statistically significant decrease in IHL content in comparison to an isocaloric control group.

Trial Registration: www.clinicaltrials.gov; NCT03067428.

Keywords: non-alcoholic fatty liver disease, hepatic steatosis, intrahepatic lipid, fructose, nutrition, dietary intervention, randomized controlled trial, glucose metabolism. 


\section{INTRODUCTION}

The drastic increase in fructose consumption since the industrial revolution has paralleled the current epidemic of obesity and its cardiometabolic complications, such as dyslipidemia, diabetes type II, non-alcoholic fatty liver disease (NAFLD) and cardiovascular disease (1-4). Furthermore, fructose overfeeding trials have convincingly shown to increase the accumulation of fat in the liver (5), the principal organ involved in the metabolism of fructose (6).

There is, however, an ongoing debate on whether fructose is more detrimental for liver health than other simple sugars, such as glucose $(6,7)$. While there is abundant evidence from animal studies on the prominent role and putative mechanism by which fructose causes IHL accumulation (8-12), a previous meta-analysis of controlled trials in humans did not show any effects of isocaloric fructose exchange for other carbohydrates on intrahepatic lipid (IHL) content or serum alanine aminotransferase levels(5). On the other hand, Schwarz and colleagues more recently demonstrated that a nine-day isocaloric fructose-restricted diet successfully reduced IHL content in obese children with high habitual sugar intake, which was mainly attributed to a decrease in de novo lipogenesis (13).

The discrepancies between these studies may be attributed to the amount of fructose that was consumed, i.e., either moderate to high (5)or very low(13). Fructose can serve as a substrate for de novo lipogenesis and stimulate de novo lipogenesis via upregulation of transcription factors that enable the expression of genes involved in de novo lipogenesis, such as sterol regulatory element binding protein 1 (SREBP1), carbohydrate response element binding protein $(\mathrm{ChREBP})$ and liver $\mathrm{X}$ receptor $(\mathrm{LXR})(14,15)$. On the other hand, even at small ('catalytic') amounts, fructose can also increase hepatic glucose disposal $(16,17)$.

As the interpretation of the intervention study by Schwarz and colleagues was limited by its single-arm design and, hence, the lack of a control group, the aim of the current study ('eFfects of fRUctose restrIcTion on LivEr SteatosiS': FRUITLESS) was to investigate the effects of fructose restriction on IHL content in a double-blind randomized controlled trial, using an isocaloric comparator. 


\section{RESEARCH DESIGN AND METHODS}

\section{Study population}

Between March 2017 and October 2019, individuals were recruited via advertisements or contacted directly if they had participated in scientific research before and agreed to be contacted for future studies. Inclusion criteria for participation were age $\geq 18$ years, a high prior chance of having an increased IHL content (i.e. a body mass index (BMI) $\geq 28 \mathrm{~kg} / \mathrm{m}^{2}$ and a fatty liver index $(\mathrm{FLI}) \geq 60(18,19))$ and a daily fructose intake above the Dutch average (i.e. $\geq 45$ grams/day (20) according to a three-day food journal). If an individual did not meet the inclusion criterion of BMI $\geq 28 \mathrm{~kg} / \mathrm{m}^{2}$, but had an elevated FLI, he/she was still considered eligible to participate. Due to a slow recruitment rate, the inclusion criterion of fructose intake $\geq 45$ grams/day was abandoned (protocol amendment July 18, 2018). Individuals were excluded from participation in case of (history of) liver disease, (history of) excessive alcohol consumption (i.e., $>3$ and $>2$ units per day for males and females, respectively), major change in weight (i.e., $>5 \%$ ) and/or physical activity pattern in the three months prior to the study, use of glucose-lowering drugs, recent illness, pregnancy and/or lactation, contraindications for magnetic resonance imaging (MRI), and inability to give informed consent. Eligibility assessment was performed by the clinical researcher of the study (N.S.).

All participants gave written informed consent prior to inclusion in the study. The study was performed according to the Declaration of Helsinki (21) and approved by the medical ethical committee of Maastricht University Medical Center. The full trial protocol can be accessed via the corresponding author or ClinicalTrials.gov (identifier: NCT03067428).

\section{Dietary intervention}

Since restriction of one nutrient (in this case fructose) without affecting other components of the diet is practically impossible, both groups were asked to follow a six-week fructoserestricted diet. In addition to this diet, the control group was supplemented with fructose powder aimed at achieving a fructose intake similar to baseline, whereas the intervention group remained fructose-restricted and received glucose powder to allow an isocaloric comparison. At screening, baseline and completion of the study, dietary intake was assessed with a threeday food journal along with a personal interview by the clinical researcher (N.S.) who was blinded to the intervention assignment. Weight of the food products was provided by the participant or estimated using average quantities per portion. Macro- and micronutrient composition and caloric content of the diet were calculated using the Dutch food composition 
table (22). Average fructose intake was calculated with an extensive database of food products from the Wageningen University (20). This database was also used for the composition of the fructose-restricted diet throughout the study. If the fructose content of a specific product was not available in the database, a comparable food product or the sucrose content ( $=50 \%$ fructose) of the specific product was used.

All participants received extensive counseling on the fructose-restricted diet by the clinical researcher of the study (N.S.) under the supervision of an experienced metabolic dietician (E.M.C.P.), who were both blinded to the intervention assignment. Participants were allowed to have an ad-libitum food intake, as long as the fructose intake per meal and per day was less than 7.5 grams and 10 grams, respectively. For this, participants were provided a list of permitted and prohibited food products as well as examples of fructose-restricted meals. Furthermore, participants received dietary counseling and diets were adjusted on a weekly basis according to the fructose intake that was assessed by personal interviews. Substantial weight loss/gain was checked every week and (if necessary) corrected with dietary advice. Randomization to either glucose or fructose supplementation was computer-generated using block sizes of four and performed by an independent researcher (M.D.G.E.). The allocation sequence was concealed in an opaque, sealed envelope and remained blinded to the participants and assessors (N.S., P.I.H.G.S., M.C.G.J.B., C.D.A.S., N.C.S., P.V. and V.B.H.S.) upon completion of all analyses. Supplementation of either glucose or fructose equaled the amount of fructose that was restricted from the diet. In case of a baseline fructose intake below the Dutch average (i.e., 45 grams/day), glucose or fructose supplementation was set at 45 grams/day. The glucose and fructose powders (indistinguishable in terms of color and odor) were prepacked in identical sachets by an independent researcher (M.D.G.E.) and distributed to the participants on a weekly basis. Participants were instructed to dissolve the glucose or fructose supplementation in water or food (e.g., yoghurt or cottage cheese) for consumption during the three main meals. If participants preferred to dissolve the glucose or fructose powder in water, they were urged to consume the supplementation solely during or directly after finishing the meal. As the six-week fructose-restricted diet is devoid of fruits and vegetables, all participants received vitamin $\mathrm{C}$ supplementation $(70 \mathrm{mg} /$ day) throughout the entire study to prevent deficiency. No additional requirements were imposed in terms of food/liquid intake or medication use. Participants were asked not to alter their physical activity throughout the study. 


\section{Primary outcome measure}

Participants were asked to visit the research ward of Maastricht University Medical Center after an 8-hour fast and to refrain from alcohol 3 days prior to the measurements. Proton magnetic resonance spectroscopy $\left({ }^{1} \mathrm{H}-\mathrm{MRS}\right)$ was performed to determine IHL content at baseline and completion. All magnetic resonance (MR) measurements were performed on a $3 \mathrm{~T}$ MR system (Achieva 3T-X Philips Healthcare, Best, Netherlands) using a 32-channel sense cardiac/torso coil (Philips Healthcare, Best, Netherlands). Due to morbid (abdominal) obesity, five participants were scanned on a wide bore system 1.5 T MR system (Ingenia, Philips Healthcare, Best, Netherlands) at both time points (i.e., baseline and follow-up measurement). A $\mathrm{T}_{2}$-weighted turbo spin echo MR image was acquired in three (axial, coronal and transversal) orientations and a $20 \times 20 \times 20 \mathrm{~mm}$ voxel was placed in the right hepatic lobe, avoiding vascular structures and edges of the liver, diaphragm and biliary structures. Special care was taken to ensure that the placement of the voxel was in same position at both time points. Hepatic lipid spectra were acquired using a PRESS sequence with water suppression (frequency selective pre-pulses) using the following MR parameters: repetition time (TR)/echo time $(\mathrm{TE})=4000 / 32.5 \mathrm{~ms}, 32$ acquisitions with 2 signal averages in each, bandwith $=2000 \mathrm{~Hz}$ and data points $=2048$. Additionally, spectra without water suppression were acquired (8 acquisitions with 2 signal averages in each) as an internal reference. The long TR was chosen to let the participant breath in the rhythm of the measurement, and MR acquisition was performed at the end of expiration. A pressure-sensitive probe was placed on the abdomen of the participant to monitor the breathing pattern throughout the measurement. All MR spectra obtained were individually frequency-aligned and -phased, and lipid- $\mathrm{CH}_{2}$ and water peaks were fitted to the respective spectrum as described before (23), using a custom-built MATLAB script (MATLAB R2017b, Mathworks, USA). The signal intensities obtained were corrected for $\mathrm{T}_{2}$ decay using the literature $\mathrm{T}_{2}$ values of $3 \mathrm{~T}(26.3 \mathrm{~ms}, 59.1 \mathrm{~ms})(24)$ and $1.5 \mathrm{~T}(50 \mathrm{~ms}, 60 \mathrm{~ms})$ (25) for water and lipid, respectively. Finally, the IHL percentage was represented as the area ratio of the $\mathrm{CH}_{2} / \mathrm{H}_{2} \mathrm{O}$ peak using the $\mathrm{T}_{2}$-corrected signal intensities of lipid- $\mathrm{CH}_{2}$ and water. Due to technical reasons, MRS was not available in one participant. IHL content of both time points was subsequently assessed using mDIXON imaging, which was validated against ${ }^{1} \mathrm{H}-\mathrm{MRS}$ in a former study $\left(\mathrm{ICC}_{\text {agreement }}=0.82 ; \mathrm{p}<0.001(26)\right)$.

\section{Secondary outcome measures}

At the same day of the ${ }^{1} \mathrm{H}-\mathrm{MRS}$ measurements, blood was drawn for the determination of serum insulin and lipids (i.e. total, HDL and LDL cholesterol and triglycerides) and a two-hour 
75-gram oral glucose tolerance test (OGTT) was performed, all exactly as described previously (27) . The area under the curve (AUC) was calculated as a measure of glucose tolerance. Insulin resistance was estimated with the HOMA2-IR calculator (www.dtu.ox.ac.uk).

\section{Other measurements}

All participants filled in a health questionnaire concerning smoking, habitual alcohol consumption and medical history. Weight was measured in solely underwear, height was assessed with a stadiometer and waist and hip circumference were determined with a measuring tape at the level of umbilicus and trochanter major, respectively. Twenty-four-hour urine was collected in pre-acidified plastic containers at $\mathrm{T}=0,2,4$ and 6 weeks for measurement of urinary fructose, an objective biomarker of fructose consumption (28), with Ultra Performance Liquid Chromatography-tandem Mass Spectrometry (UPLC-MS/MS; VC\% 6.6)).

\section{Statistical analysis}

For this double-blind randomized controlled trial, sample size calculation showed that 19 individuals per group were required to detect a mean difference of $3 \% \pm 3.2 \%$ in IHL content $\left(\alpha=0.05, \beta=0.20\right.$, allocation ratio $\left.\mathrm{N}_{\text {intervention group }}: \mathrm{N}_{\text {control group }}=1: 1\right)$. To account for drop-out $(15 \%)$, the number of individuals was increased to 22 per group.

Dichotomous data are expressed as frequencies. Continuous data are presented as median (interquartile range). Changes from baseline within and between groups were analyzed with a Wilcoxon signed-rank test and a Mann Whitney $U$ test, respectively. The 95\% confidence intervals $(95 \% \mathrm{CI})$ for these changes were calculated according to the Hodges-Lehmann method.

Sensitivity analyses were conducted 1) to assess the influence of unbalanced randomization, if present, on the primary outcome measure. For this, a one-way ANCOVA was conducted with the unbalanced variable as a covariate; 2) to determine the effect of the protocol amendment (i.e. the omission of the fructose intake $\geq 45$ grams/day inclusion criterion) on the primary outcome measure; 3 ) to assess the effect of the degree of IHL content at baseline (i.e. above or below the $5.56 \%$ cut-off value (25)) on the primary outcomes measure. For both analyses, interaction terms were tested in a one-way ANCOVA; and 4) to explore a dose response relationship by testing the association between the change in fructose intake and the change in IHL content and by testing for an interaction between the intervention and the change in fructose intake on the change in IHL content (one-way ANCOVA). 


\section{$\underline{\text { CHAPTER } 5}$}

A p-value $<0.05$ was considered statistically significant. All analyses were carried out with the IBM Statistical Package of Social Sciences (SPSS) version 25 for Windows (SPSS inc. Chicago, IL).

\section{RESULTS}

\section{Randomization and follow-up}

Forty-four participants were randomly assigned to the intervention group (fructose-restricted diet plus glucose supplementation) or control group (fructose-restricted diet plus fructose supplementation) (see Figure 1 for flow chart). Six participants discontinued participation because of various reasons (i.e., claustrophobia during MR measurements ( $n=2)$, nonadherence to the diet (as indicated by the participant) $(n=1)$, immobility due to ankle distortion $(n=1)$, symptoms related to irritable bowel syndrome (before start of the diet) $(n=1)$ and personal reasons $(n=1))$. At completion of the study, one participant did not appear to meet the inclusion criteria (i.e., FLI $\geq 60$ ) and was therefore excluded from the analyses. The individualized supplementation was successfully supplied and ingested by all participants. Since follow-up of the primary outcome could not be acquired for the six individuals who discontinued participation, the intention-to-treat analyses did not differ from the per protocol analyses, which were carried out with 16 and 21 participants in the intervention and control group, respectively. Baseline characteristics are displayed in Table 1.

\section{Table 1 General baseline characteristics of the study population}

\begin{tabular}{l|c|c}
\hline Characteristic & Intervention group (n=16) & Control group (n=21) \\
\hline Age (yr) & $55(35-62)$ & $52(38-62)$ \\
Sex (n, M/F) & $6 / 10$ & $6 / 15$ \\
Smoking (\% yes) & 12.5 & 14.3 \\
Alcohol intake (units/week) & $2(1-5)$ & $3(0-5)$ \\
BMI (kg/m2) & $34.1(28.8-37.3)$ & $31.1(30.2-35.6)$ \\
Waist circumference (cm) & $117.9(106.5-128.4)$ & $110.0(104.3-113.6)$ \\
HOMA2-IR & $0.84(0.50-1.37)$ & $0.86(0.73-1.14)$ \\
Serum total cholesterol (mmol/L) & $5.2(4.3-5.6)$ & $5.2(4.2-6.0)$ \\
Serum HDL-cholesterol (mmol/L) & $1.2(1.1-1.3)$ & $1.3(1.0-1.5)$ \\
Serum LDL-cholesterol (mmol/L) & $3.1(2.3-3.8)$ & $2.7(2.3-3.8)$ \\
Serum triglycerides (mmol/L) & $1.6(1.2-1.9)$ & $1.3(0.9-1.7)$ \\
Dietary fructose intake (g/day) & $42.1(20.3-73.4)$ & $36.9(27.1-54.6)$ \\
Fatty liver index & $94(74-96)$ & $84(64-91)$ \\
Intrahepatic lipid content (\%) & $4.9(2.3-10.3)$ & $2.1(0.9-7.7)$ \\
\hline
\end{tabular}

Data are expressed as median (interquartile range). Abbreviations: high-density lipoprotein (HDL), homeostasis model assessment of insulin resistance (HOMA2-IR), low-density lipoprotein (LDL), years (yr). 


\section{Enrollment}

Assessed for eligibility $(\mathrm{n}=71)$

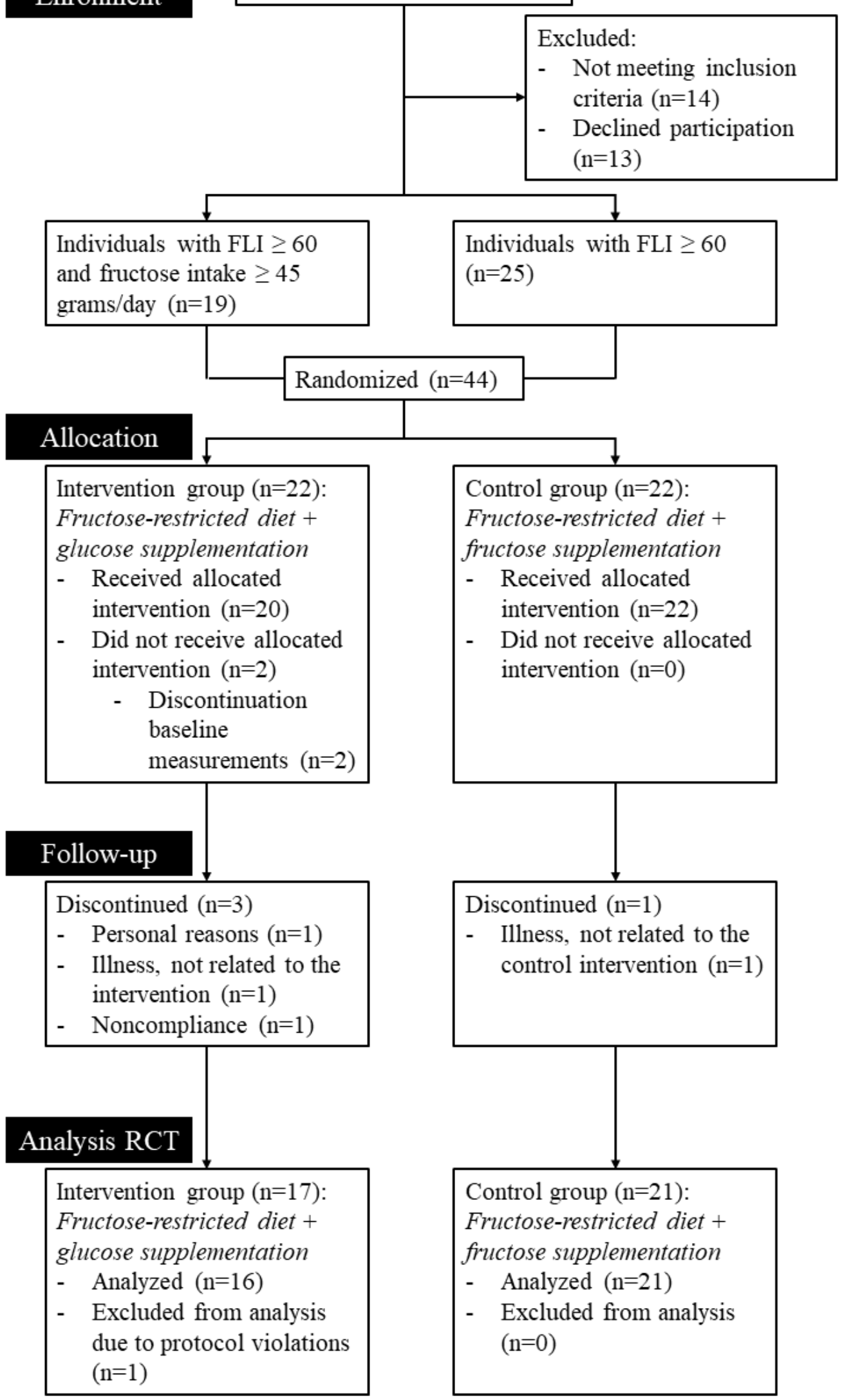

Figure 1: Flow chart of the study. 


\section{$\underline{\text { CHAPTER } 5}$}

\section{Adherence and tolerability of the intervention}

Compared to baseline, the habitual dietary intake of fructose decreased drastically in both groups (Figure 2, Panel A). The median dietary fructose intake after six weeks of fructose restriction was 1.4 grams/day in the intervention group and 2.2 grams/day in the control group (change from baseline: -45.7 grams/day, 95\%CI: -61.4, -29.8 and -37.9 grams/day, 95\%CI: $48.3,-28.6$, respectively).

A

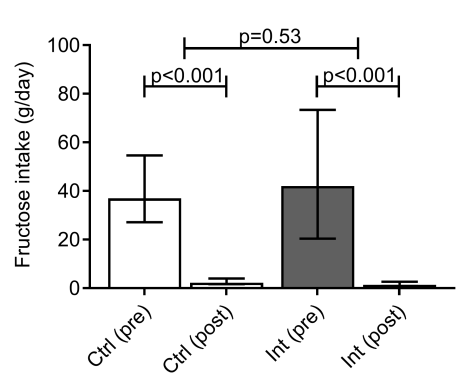

$\mathrm{D}$

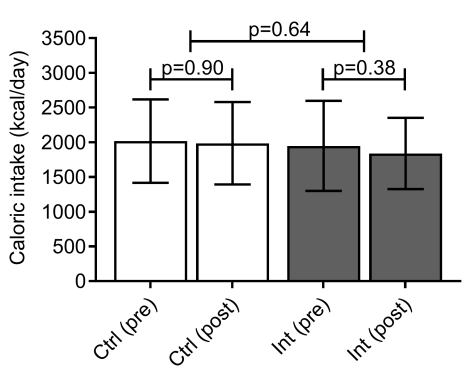

B

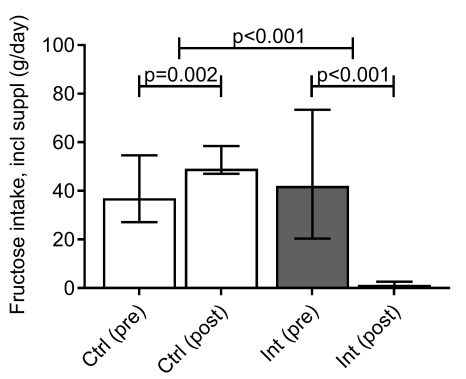

$\mathrm{E}$

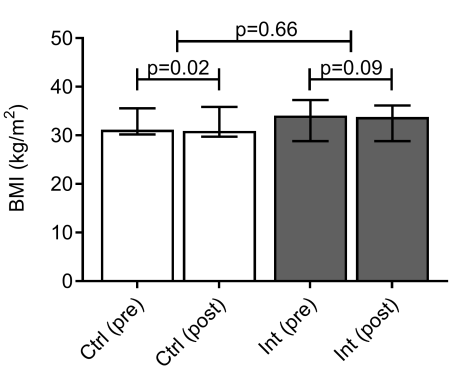

C

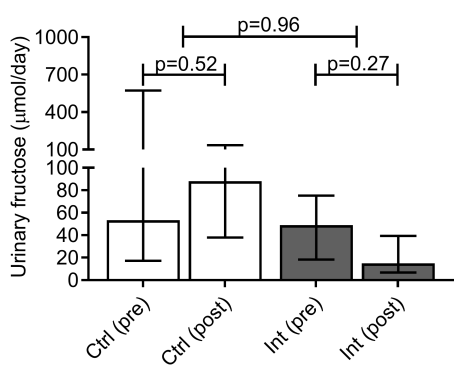

Figure 2: Adherence to the intervention. Daily fructose intake (A), daily fructose intake including supplementation (B), 24-hour urinary fructose concentration (C), daily caloric intake (D) and body mass index (BMI) (E) in the control (Ctrl; white bars, $n=21$ ) and intervention (Int; grey bars, $n=16$ ) group at baseline (pre) and after completion of the study (post). Data are expressed as median \pm interquartile range. Differences within groups are analyzed with a Wilcoxon signed-rank test. Differences between groups are analyzed with a Mann Whitney U test.

The change from baseline was not significantly different between both groups (difference between change from baseline: -6.7 grams/day, 95\%CI: -24.0, 13.0). When the supplementation was taken into account, the fructose intake in the control group increased to 49.1 grams per day (change from baseline: +12.4 grams/day, 95\%CI: 6.2, 20.8). This was higher than baseline, since supplementation of fructose (and glucose) was set at 45 grams/day in all participants with a baseline fructose intake below the Dutch average (see Research Design and Methods section). This change was significantly different from the intervention group (difference between change from baseline: -57.0 grams/day, 95\%CI: -77.9, -39.5) (Figure 2, 
Panel B; Supplementary Figure 1). The cumulative difference in fructose intake during the full study period - calculated as the individual difference from baseline times the number of days each participant was included in the study - was 1,894 grams $(95 \% \mathrm{CI}$ : 1,727, 2,052). This difference was also reflected by a significantly different urinary fructose excretion between the intervention and control group at completion of the study $(-38.8 \mu \mathrm{mol} / \mathrm{day}, 95 \% \mathrm{CI}$ : -91.2, -10.7). The change from baseline between the groups was not significantly different, which was mainly attributed to the large variation in urinary fructose concentration at baseline in the control group (Figure 2, Panel C).

Caloric intake remained stable throughout the intervention as a consequence of isocaloric supplementation and did not differ between the two groups (Figure 2, Panel D). Nevertheless, BMI decreased in both the intervention and control group (change from baseline: $-0.2 \mathrm{~kg} / \mathrm{m} 2$, 95\%CI: $-0.5,0.0$ and $-0.4 \mathrm{~kg} / \mathrm{m} 2,95 \% \mathrm{CI}:-0.6,-0.1$, respectively), which was not significantly different between both groups (difference between change from baseline: $-0.1 \mathrm{~kg} / \mathrm{m} 2,95 \% \mathrm{CI}$ : $-0.3,0.5$, Figure 2, Panel E). No statistically significant effect was observed for macronutrient composition (i.e., carbohydrate, protein, total fat and saturated fat intake, Supplementary Figure 2, Panel A-D). When macronutrient composition was expressed as percentage of total daily energy intake, a significant difference between change from baseline was observed for carbohydrate intake (difference between change from baseline: 7.1\%, 95\% CI: 0.7, 14.3, Supplementary Figure 3, panel A), which appeared to be in exchange for protein intake (difference between change from baseline: -5.9\%, 95\%CI: -13.3, 0.4, Supplementary Figure 2, panel B).

The intervention was generally well tolerated. Diarrhea (grade 1-2) was reported in three and five participants in the intervention and control group, respectively. Only one participant in the control group experienced a serious adverse event, which occurred between the screening visit and baseline measurements.

\section{Effect of fructose restriction on intrahepatic lipid content}

IHL content decreased in both treatment arms (Figure 3, Panel A). The reduction in IHL content was significantly greater in the intervention group, albeit small (difference between change from baseline: $-0.7 \%, 95 \% \mathrm{CI}$ : -2.0, -0.03) (Figure 3, Panel A). Since baseline BMI and IHL content tended to be higher in the intervention group (Table 1), we subsequently conducted a sensitivity analysis to assess the impact thereof on the primary outcome measure. 


\section{$\underline{\text { CHAPTER } 5}$}

A

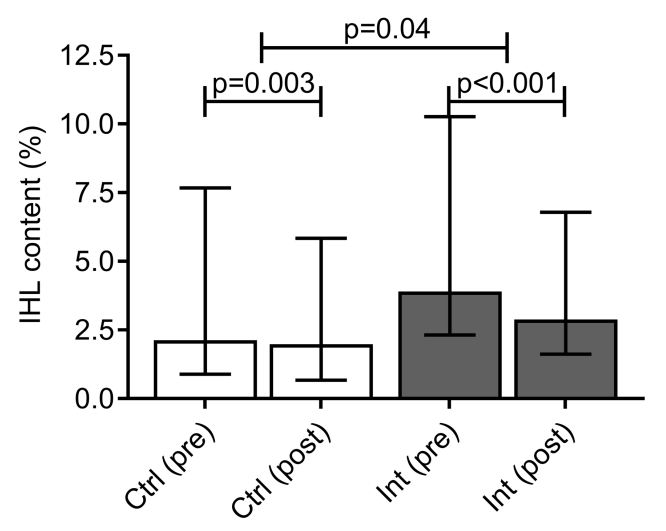

C

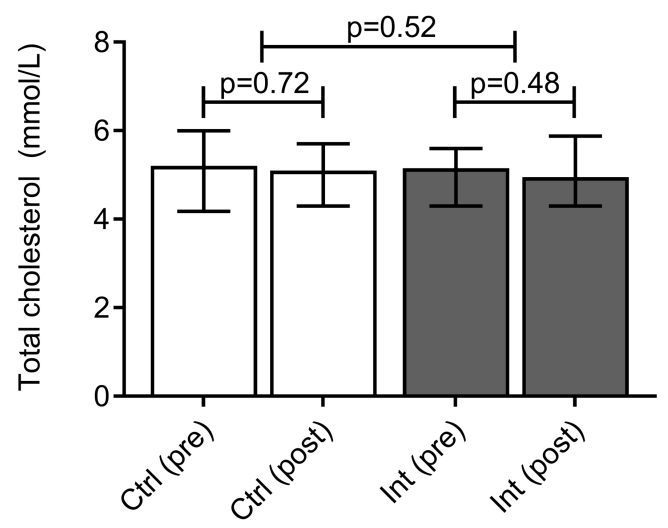

$E$

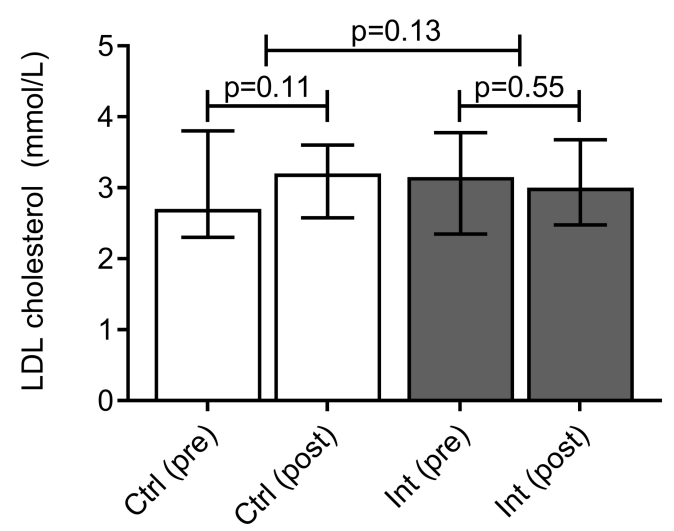

B
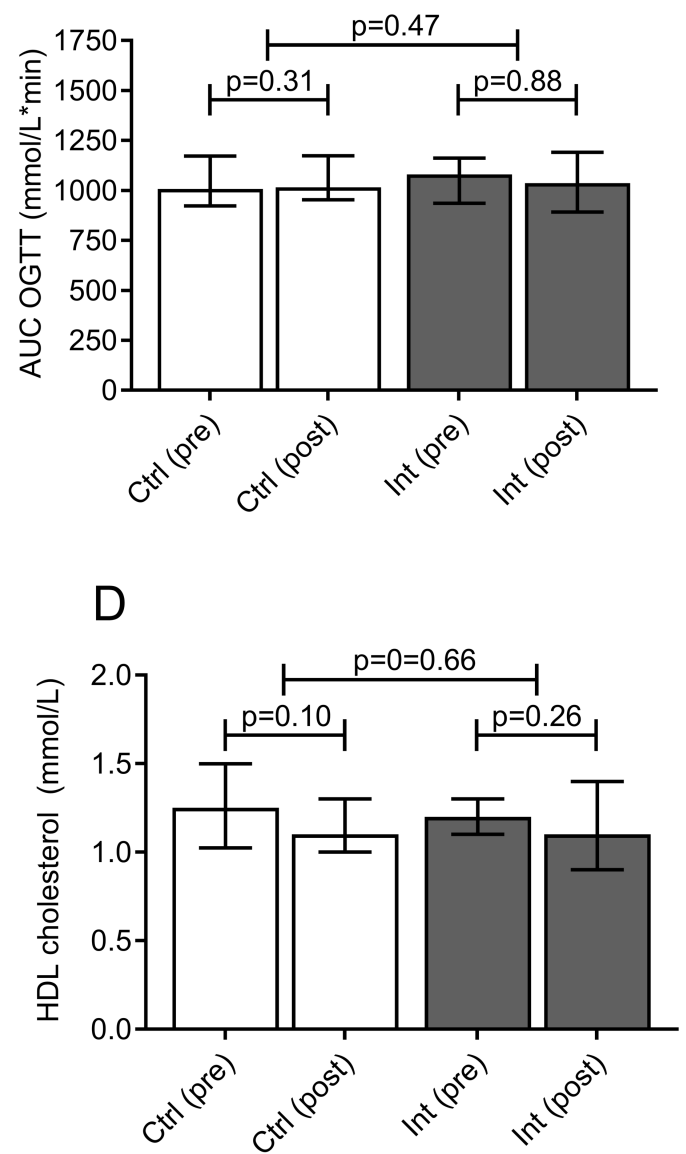

$\mathrm{F}$

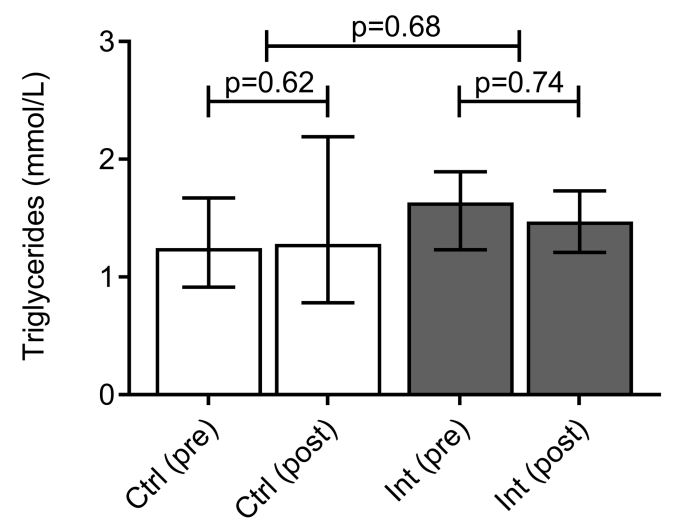

Figure 3: Intrahepatic lipid content, glucose tolerance and serum lipid levels. Intrahepatic lipid content (A), glucose area under the curve (AUC) during an oral glucose tolerance test (OGTT) (B), serum total cholesterol (C), serum HDL cholesterol (D), serum LDL cholesterol (E) and serum triglycerides $(\mathrm{F})$ in the control ( $\mathrm{Ctrl}$; white bars, $\mathrm{n}=21$ ) and intervention (Int; grey bars, $\mathrm{n}=16)$ group at baseline (pre) and after completion of the study (post). Data are expressed as median \pm interquartile range. Differences within groups are analyzed with a Wilcoxon signed-rank test. Differences between groups are analyzed with a Mann Whitney U test. 
Addition of baseline BMI and IHL content as covariates to a one-way ANCOVA did not have substantial effects on the (log-transformed) primary outcome measure (beta: $-0.06,95 \% \mathrm{CI}$ : $0.116,0.007, \mathrm{p}=0.08$ ). Subgroup analyses did not show significantly different effect sizes between participants with either high or low habitual fructose intake, or high or low baseline IHL content ( $\mathrm{p}$ for interaction: 0.63 and 0.75, respectively; Supplementary Figure 4). Finally, the change in fructose intake was not associated with the change in IHL content $(p=0.34)$, nor was there an interaction between the intervention and the change in fructose intake on the change in IHL content $(\mathrm{p}=0.72)$.

\section{Effect of fructose restriction on glucose tolerance and serum lipid levels}

Plasma glucose excursions during a two-hour 75-gram OGTT were not significantly different between the two groups (difference between change from baseline: $32.3,95 \% \mathrm{CI}$ : $-62.3,119.0$, Figure 3, Panel B). HOMA2-IR was neither affected in the intervention nor in the control group (change from baseline:0.12, 95\%CI: $-0.17,0.51$ and $0.06,95 \% \mathrm{CI}$ : $-0.13,0.19$, respectively), nor was there a difference between the groups (difference between change from baseline: 0.10 , 95\%CI: $-0.21,0.42$ ). Additionally, no effects were observed on serum lipids (Figure 3, Panel C-F).

\section{DISCUSSION}

In this double-blind randomized controlled trial, we show that a six-week fructose-restricted diet resulted in a small decrease in IHL content in adult overweight individuals with a high FLI in comparison to an isocaloric comparator. No effects were observed on glucose tolerance or serum lipid levels.

Although glucose and fructose are both simple sugars with identical molecular formulas, their metabolic fate differs greatly. In contrast to glucose, which is widely metabolized throughout the body, a proportion of ingested fructose is already cleared in the small intestine, as shown by animal studies (29). After absorption, fructose reaches the portal blood where it is almost entirely taken up by the liver to be converted to glucose, lactate, glycogen or triglycerides (30). Furthermore, at a low dose, fructose facilitates hepatic glucose disposal by dissociation of the glucokinase-glucokinase regulatory protein complex (GCK-GKRP) (16,31). Dissociation of this complex appears clinically relevant, since carriers of a missense variant in GCKR - the gene that encodes a GKRP protein that binds GCK less effectively - are prone to develop NAFLD (32). Thus, although clear metabolic differences between fructose and glucose 


\section{$\underline{\text { CHAPTER } 5}$}

metabolism exist, such differences cannot be easily extrapolated to net effects on metabolic health, including IHL content, and experiments such as the current trial may provide important insights.

Dose-dependent metabolic pathways as described above may also explain the conflicting results of previous clinical trials $(5,13)$. To date, fructose intervention studies have mainly focused on the role of fructose added to the diet and showed contrasting effects on IHL content (5,33-36), Recently, Schwarz and colleagues (13) showed that restriction of fructose from the diet reduced IHL content in obese children and adolescents. The lack of a control group, however, makes it difficult to conclude whether this change in IHL content can be attributed to fructose restriction per se, or alternatively, to the change in the diet in general or other behaviors that are associated with participation in a clinical trial (i.e. the Hawthorne effect (37)).

The current study is the first that used a randomized controlled study design in which both the intervention and control group followed the same fructose-restricted diet. Together with a similar caloric intake in both treatment arms (as a consequence of the isocaloric glucose supplementation in the intervention group), we were able to study the effect of fructose restriction per se. Indeed, the results in the control group show that the diet by itself was already associated with a decrease in IHL content, independent of a change in fructose intake (which was supplemented). More importantly, by comparing both treatment arms we are able to show that fructose restriction per se reduces IHL content, albeit with a small effect size $(0.7$ percent point).

In the present study, we did not observe any effect of fructose restriction on serum lipid levels, glucose tolerance or HOMA2-IR, which was somewhat surprising since these variables have been associated with IHL content $(38,39)$. A possible explanation might be that the effect of fructose restriction on IHL content was too small to result in a detectable change in serum lipid levels and/or glucose tolerance. In addition, we did not assess insulin sensitivity with the gold standard method, i.e., a hyperinsulinemic-euglycemic clamp. Another possible explanation might be that the duration of the intervention was too short. Yet, this period is comparable to most other fructose feeding trials, which varied between the three and ten weeks (40). Moreover, it has been shown that IHL accumulation can resolve within days $(13,41)$. We, therefore, believe that a six-week intervention period - in which the cumulative difference in 
fructose consumption was almost 2 kilograms - is sufficient to reach a steady state in these metabolic outcomes.

Our study has strengths and limitations. First, the sample size was relatively small, but should be viewed in perspective of the major dietary restrictions that were imposed. More importantly, our study was adequately powered with an expected drop-out rate. Second, we used the FLI as a screening tool for fatty liver (42). Despite a high positive likelihood ratio to rule in fatty liver with a FLI $\geq 60$ (43-45), the median IHL content of the study participants was only $3.4 \%$. This was not attributable to regression to the mean, since FLI was high at both the screening and baseline visit (91 versus 87, respectively). Nevertheless, sensitivity analysis with stratification for high and low liver fat showed that similar effects sizes were observed in individuals with a high IHL content at baseline. Furthermore, we did not observe a dose response relationship between the change in fructose intake and the change in IHL content. It should, however, be noted that this study was not designed and hence, not powered to assess a dose response relationship. Third, due to a slow inclusion rate we decided to abandon the inclusion criterion of fructose intake $\geq 45$ grams/day. To safeguard a detectable effect on IHL content, the supplementation of either glucose or fructose was increased to 45 grams/day in participants with a low habitual fructose intake. Again, a sensitivity analysis did not show any significant difference when we stratified for fructose intake at baseline. Fourth, supplemented fructose may not have the same metabolic effects as fructose containing food products. The food matrix may play a role in the timing of fructose absorption - in the current study the supplemented fructose was dissolved in water or food - and may cause an interaction between different nutrients. For instance, it has been postulated that that the presence of vitamin $\mathrm{C}$ in fruit and vegetables abolishes the toxic effects of fructose (46-48). Fifth, despite major efforts to achieve an isocaloric diet in comparison to the habitual baseline diet of participants, BMI decreased similarly in both the intervention and control group, which could be explained by the Hawthorne effect.

A major strength of this study is the double-blind randomized controlled design, using an isocaloric comparator. As hitherto mentioned, both groups followed the same fructoserestricted diet, which is of importance, given the differential impact of macronutrients on IHL content $(49,50)$. Despite this design, we did find some small, probably accidental differences in carbohydrate intake expressed as percentage of total energy intake (i.e., relatively higher in the intervention group), which tended to be in exchange for protein intake (i.e., relatively lower 


\section{$\underline{\text { CHAPTER } 5}$}

in the intervention group). Given the previously described effects of carbohydrates and proteins on IHL content (i.e. predisposing and protective, respectively) $(49,50)$, the effects of these subtle differences in macronutrient composition on IHL content, if any, have probably mitigated the observed difference in the current study. Finally, not only did we used a threeday food journal combined with personal interviews to assess fructose intake throughout the study, we were also able to objectively confirm adherence to the study protocol by measuring urinary fructose levels, a biomarker of fructose intake (28).

\section{CONCLUSION}

In this double-blind randomized controlled trial, we showed that six-week fructose-restriction per se results in a small but statistically significant decrease in IHL content in overweight adult individuals with a high FLI in comparison to an isocaloric control group. No effects were observed on glucose tolerance or serum lipid levels.

\section{ACKNOWLEDGEMENTS}

We thank Jean L.J.M. Scheijen, Amée M. Buziau, Petra M. Niessen, Jos Op 't Roodt, Yvo H.A.M. Kusters and Armand M.A. Linkens (Department of Internal Medicine, Maastricht University Medical Center, Maastricht, The Netherlands) for their assistance during the execution of the study. This study was supported by research grants from Nutricia Research Foundation (grant 2016-33) and the Netherlands Heart Foundation (grant 2015T042). The authors confirm independence from the sponsors; the content of the article has not been influenced by the sponsors. The authors have declared that no conflicts of interests exist. 


\section{REFERENCES}

1. Bray GA, Nielsen SJ, Popkin BM. Consumption of high-fructose corn syrup in beverages may play a role in the epidemic of obesity. Am J Clin Nutr. 2004 Apr;79(4):537-43.

2. Schulze MB, Manson JE, Ludwig DS, Colditz GA, Stampfer MJ, Willett WC, et al. Sugar-sweetened beverages, weight gain, and incidence of type 2 diabetes in young and middle-aged women. JAMA. 2004 Aug 25;292(8):927-34.

3. Jensen T, Abdelmalek MF, Sullivan S, Nadeau KJ, Green M, Roncal C, et al. Fructose and sugar: A major mediator of non-alcoholic fatty liver disease. J Hepatol. 2018 May;68(5):1063-75.

4. Yang Q, Zhang Z, Gregg EW, Flanders WD, Merritt R, Hu FB. Added sugar intake and cardiovascular diseases mortality among US adults. JAMA Intern Med. 2014 Apr;174(4):516-24.

5. Chiu S, Sievenpiper JL, de Souza RJ, Cozma AI, Mirrahimi A, Carleton AJ, et al. Effect of fructose on markers of non-alcoholic fatty liver disease (NAFLD): a systematic review and meta-analysis of controlled feeding trials. Eur J Clin Nutr. 2014 Apr;68(4):416-23.

6. Hannou SA, Haslam DE, McKeown NM, Herman MA. Fructose metabolism and metabolic disease. J Clin Invest. 2018 Feb 1;128(2):545-55.

7. Sievenpiper JL, de Souza RJ, Jenkins DJA. Sugar: fruit fructose is still healthy. Nature. 2012 Feb;482(7386):470-470.

8. Ackerman Z, Oron-Herman M, Grozovski M, Rosenthal T, Pappo O, Link G, et al. Fructose-induced fatty liver disease: hepatic effects of blood pressure and plasma triglyceride reduction. Hypertens Dallas Tex 1979. 2005 May;45(5):1012-8.

9. Armutcu F, Coskun O, Gürel A, Kanter M, Can M, Ucar F, et al. Thymosin alpha 1 attenuates lipid peroxidation and improves fructose-induced steatohepatitis in rats. Clin Biochem. 2005 Jun;38(6):540-7.

10. Koo H-Y, Miyashita M, Cho BHS, Nakamura MT. Replacing dietary glucose with fructose increases ChREBP activity and SREBP-1 protein in rat liver nucleus. Biochem Biophys Res Commun. 2009 Dec $11 ; 390(2): 285-9$.

11. Roglans N, Vilà L, Farré M, Alegret M, Sánchez RM, Vázquez-Carrera M, et al. Impairment of hepatic Stat3 activation and reduction of PPARalpha activity in fructose-fed rats. Hepatol Baltim Md. 2007 Mar;45(3):778-88.

12. Samuel VT, Liu Z-X, Wang A, Beddow SA, Geisler JG, Kahn M, et al. Inhibition of protein kinase Cepsilon prevents hepatic insulin resistance in nonalcoholic fatty liver disease. J Clin Invest. 2007 Mar; 117(3):73945.

13. Schwarz J-M, Noworolski SM, Erkin-Cakmak A, Korn NJ, Wen MJ, Tai VW, et al. Effects of Dietary Fructose Restriction on Liver Fat, De Novo Lipogenesis, and Insulin Kinetics in Children with Obesity. Gastroenterology. 2017 Sep;153(3):743-52.

14. Gluchowski NL, Becuwe M, Walther TC, Farese RV. Lipid droplets and liver disease: from basic biology to clinical implications. Nat Rev Gastroenterol Hepatol. 2017 Jun;14(6):343-55.

15. DiStefano JK. Fructose-mediated effects on gene expression and epigenetic mechanisms associated with NAFLD pathogenesis. Cell Mol Life Sci CMLS. 2020 Jun;77(11):2079-90.

16. Petersen KF, Laurent D, Yu C, Cline GW, Shulman GI. Stimulating effects of low-dose fructose on insulinstimulated hepatic glycogen synthesis in humans. Diabetes. 2001 Jun;50(6):1263-8. 


\section{CHAPTER 5}

17. Buziau AM, Schalkwijk CG, Stehouwer CDA, Tolan DR, Brouwers MCGJ. Recent advances in the pathogenesis of hereditary fructose intolerance: implications for its treatment and the understanding of fructose-induced non-alcoholic fatty liver disease. Cell Mol Life Sci CMLS. 2020 May;77(9):1709-19.

18. Li L, Liu D-W, Yan H-Y, Wang Z-Y, Zhao S-H, Wang B. Obesity is an independent risk factor for nonalcoholic fatty liver disease: evidence from a meta-analysis of 21 cohort studies. Obes Rev Off J Int Assoc Study Obes. 2016 Jun;17(6):510-9.

19. Bedogni G, Bellentani S, Miglioli L, Masutti F, Passalacqua M, Castiglione A, et al. The Fatty Liver Index: a simple and accurate predictor of hepatic steatosis in the general population. BMC Gastroenterol. 2006 Nov 2;6:33.

20. Sluik D, Engelen AI, Feskens EJ. Fructose consumption in the Netherlands: the Dutch National Food Consumption Survey 2007-2010. Eur J Clin Nutr. 2015 Apr;69(4):475-81.

21. World Medical Association. Ethical principles for medical research involving human subjects. Eur J Emerg Med Off J Eur Soc Emerg Med. 2001 Sep;8(3):221-3.

22. Rijksinstituut voor Volksgezondheid en Milieu [National Institute for Public Health and the Environment]. NEVO-table [Dutch Food Composition table]. In Nederlands Voedingsstoffenbestand 2010 [Dutch Food Composition Database 2010] Den Haag:| RIVM; 2010

23. Lindeboom L, Nabuurs CI, Hesselink MKC, Wildberger JE, Schrauwen P, Schrauwen-Hinderling VB. Proton magnetic resonance spectroscopy reveals increased hepatic lipid content after a single high-fat meal with no additional modulation by added protein. Am J Clin Nutr. 2015 Jan;101(1):65-71.

24. Guiu B, Petit J-M, Loffroy R, Ben Salem D, Aho S, Masson D, et al. Quantification of liver fat content: comparison of triple-echo chemical shift gradient-echo imaging and in vivo proton MR spectroscopy. Radiology. 2009 Jan;250(1):95-102.

25. Szczepaniak LS, Nurenberg P, Leonard D, Browning JD, Reingold JS, Grundy S, et al. Magnetic resonance spectroscopy to measure hepatic triglyceride content: prevalence of hepatic steatosis in the general population. Am J Physiol Endocrinol Metab. 2005 Feb;288(2):E462-468.

26. Kusters YH, Schalkwijk CG, Houben AJ, Kooi ME, Lindeboom L, Op 't Roodt J, et al. Independent tissue contributors to obesity-associated insulin resistance. JCI Insight. 2017 Jul 6;2(13).

27. Simons N, Debray F-G, Schaper NC, Kooi ME, Feskens EJM, Hollak CEM, et al. Patients With Aldolase B Deficiency Are Characterized by Increased Intrahepatic Triglyceride Content. J Clin Endocrinol Metab. 2019 Nov 1;104(11):5056-64.

28. Tasevska N, Runswick SA, McTaggart A, Bingham SA. Urinary sucrose and fructose as biomarkers for sugar consumption. Cancer Epidemiol Biomark Prev Publ Am Assoc Cancer Res Cosponsored Am Soc Prev Oncol. 2005 May;14(5):1287-94.

29. Jang C, Hui S, Lu W, Cowan AJ, Morscher RJ, Lee G, et al. The Small Intestine Converts Dietary Fructose into Glucose and Organic Acids. Cell Metab. 2018 Feb 6;27(2):351-361.e3.

30. Sun SZ, Empie MW. Fructose metabolism in humans - what isotopic tracer studies tell us. Nutr Metab. 2012 Oct 2;9(1):89.

31. Shiota M, Moore MC, Galassetti P, Monohan M, Neal DW, Shulman GI, et al. Inclusion of low amounts of fructose with an intraduodenal glucose load markedly reduces postprandial hyperglycemia and hyperinsulinemia in the conscious dog. Diabetes. $2002 \mathrm{Feb} ; 51(2): 469-78$.

32. Speliotes EK, Yerges-Armstrong LM, Wu J, Hernaez R, Kim LJ, Palmer CD, et al. Genome-wide association analysis identifies variants associated with nonalcoholic fatty liver disease that have distinct effects on metabolic traits. PLoS Genet. 2011 Mar;7(3):e1001324. 
33. Sobrecases H, Lê K-A, Bortolotti M, Schneiter P, Ith M, Kreis R, et al. Effects of short-term overfeeding with fructose, fat and fructose plus fat on plasma and hepatic lipids in healthy men. Diabetes Metab. 2010 Jun;36(3):244-6.

34. Lê K-A, Ith M, Kreis R, Faeh D, Bortolotti M, Tran C, et al. Fructose overconsumption causes dyslipidemia and ectopic lipid deposition in healthy subjects with and without a family history of type 2 diabetes. Am $\mathrm{J}$ Clin Nutr. 2009 Jun;89(6):1760-5.

35. Kechagias S, Ernersson A, Dahlqvist O, Lundberg P, Lindström T, Nystrom FH, et al. Fast-food-based hyperalimentation can induce rapid and profound elevation of serum alanine aminotransferase in healthy subjects. Gut. 2008 May;57(5):649-54.

36. Sevastianova K, Santos A, Kotronen A, Hakkarainen A, Makkonen J, Silander K, et al. Effect of short-term carbohydrate overfeeding and long-term weight loss on liver fat in overweight humans. Am J Clin Nutr. 2012 Oct;96(4):727-34.

37. McCarney R, Warner J, Iliffe S, van Haselen R, Griffin M, Fisher P. The Hawthorne Effect: a randomised, controlled trial. BMC Med Res Methodol. 2007 Jul 3;7:30.

38. DeFilippis AP, Blaha MJ, Martin SS, Reed RM, Jones SR, Nasir K, et al. Nonalcoholic fatty liver disease and serum lipoproteins: The Multi-Ethnic Study of Atherosclerosis. Atherosclerosis. 2013 Apr;227(2):42936.

39. Wang B, Li M, Zhao Z, Wang S, Lu J, Chen Y, et al. Glycemic Measures and Development and Resolution of Nonalcoholic Fatty Liver Disease in Nondiabetic Individuals. J Clin Endocrinol Metab. 2020 May $1 ; 105(5)$.

40. Sievenpiper JL, de Souza RJ, Cozma AI, Chiavaroli L, Ha V, Mirrahimi A. Fructose vs. glucose and metabolism: do the metabolic differences matter? Curr Opin Lipidol. 2014 Feb;25(1):8-19.

41. Bortolotti M, Kreis R, Debard C, Cariou B, Faeh D, Chetiveaux M, et al. High protein intake reduces intrahepatocellular lipid deposition in humans. Am J Clin Nutr. 2009 Oct;90(4):1002-10.

42. European Association for the Study of the Liver (EASL), European Association for the Study of Diabetes (EASD), European Association for the Study of Obesity (EASO). EASL-EASD-EASO Clinical Practice Guidelines for the management of non-alcoholic fatty liver disease. J Hepatol. 2016 Jun;64(6):1388-402.

43. Yang B-L, Wu W-C, Fang K-C, Wang Y-C, Huo T-I, Huang Y-H, et al. External validation of fatty liver index for identifying ultrasonographic fatty liver in a large-scale cross-sectional study in Taiwan. PloS One. 2015;10(3):e0120443.

44. Cuthbertson DJ, Weickert MO, Lythgoe D, Sprung VS, Dobson R, Shoajee-Moradie F, et al. External validation of the fatty liver index and lipid accumulation product indices, using $1 \mathrm{H}$-magnetic resonance spectroscopy, to identify hepatic steatosis in healthy controls and obese, insulin-resistant individuals. Eur J Endocrinol. 2014 Nov;171(5):561-9.

45. Koehler EM, Schouten JNL, Hansen BE, Hofman A, Stricker BH, Janssen HLA. External validation of the fatty liver index for identifying nonalcoholic fatty liver disease in a population-based study. Clin Gastroenterol Hepatol Off Clin Pract J Am Gastroenterol Assoc. 2013 Sep;11(9):1201-4.

46. Lanaspa MA, Sanchez-Lozada LG, Choi Y-J, Cicerchi C, Kanbay M, Roncal-Jimenez CA, et al. Uric acid induces hepatic steatosis by generation of mitochondrial oxidative stress: potential role in fructosedependent and -independent fatty liver. J Biol Chem. 2012 Nov 23;287(48):40732-44.

47. Kc S, Cárcamo JM, Golde DW. Vitamin C enters mitochondria via facilitative glucose transporter 1 (Glut1) and confers mitochondrial protection against oxidative injury. FASEB J Off Publ Fed Am Soc Exp Biol. 2005 Oct;19(12):1657-67. 


\section{CHAPTER 5}

48. Johnson RJ, Stenvinkel P, Andrews P, Sánchez-Lozada LG, Nakagawa T, Gaucher E, et al. Fructose metabolism as a common evolutionary pathway of survival associated with climate change, food shortage and droughts. J Intern Med. 2020 Mar;287(3):252-62.

49. Luukkonen PK, Sädevirta S, Zhou Y, Kayser B, Ali A, Ahonen L, et al. Saturated Fat Is More Metabolically Harmful for the Human Liver Than Unsaturated Fat or Simple Sugars. Diabetes Care. 2018 Aug;41(8):1732-9.

50. Markova M, Pivovarova O, Hornemann S, Sucher S, Frahnow T, Wegner K, et al. Isocaloric Diets High in Animal or Plant Protein Reduce Liver Fat and Inflammation in Individuals with Type 2 Diabetes. Gastroenterology. 2017 Feb;152(3):571-585.e8. 


\section{SUPPLEMENTARY DATA}

A

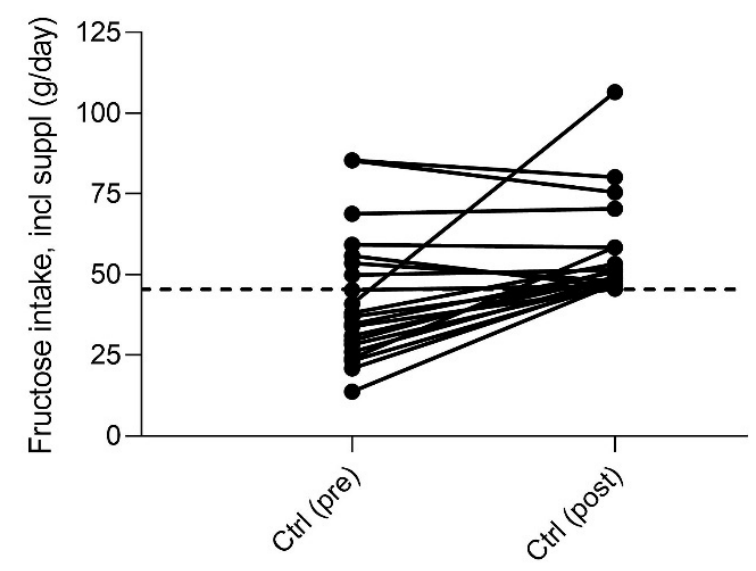

B

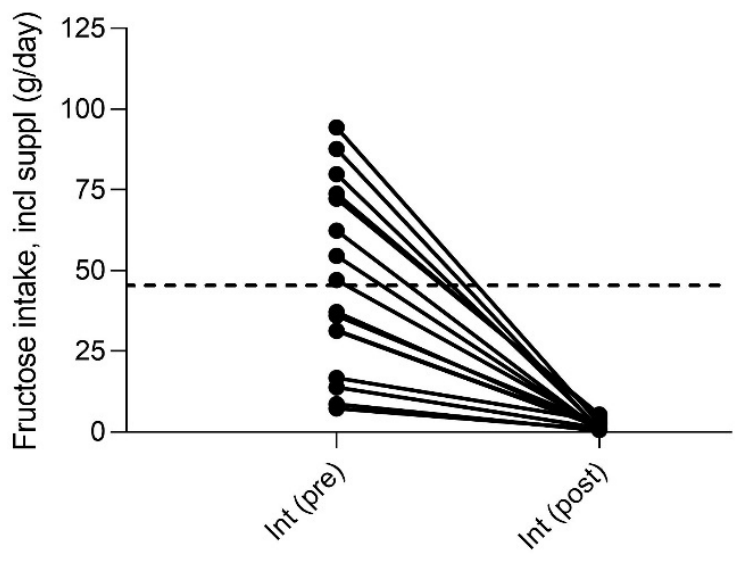

Supplementary figure 1: Individual fructose intake pre- and post-intervention. Fructose intake at baseline (pre) and after completion of the study (post) in the control (Ctrl; panel A, n=21) and intervention (Int; panel B, $\mathrm{n}=16$ ) group. Data are expressed as individual data points. All participants followed a fructose-restricted diet. Individuals who consumed $\geq 45$ grams of fructose per day at baseline (i.e., above the dashed horizontal line) received supplementation of fructose (control group, panel A) or glucose (intervention group, panel B) in isocaloric exchange for the amount of fructose that was restricted from the diet. Individuals who consumed $<45$ grams of fructose per day at baseline (i.e., below the dashed horizontal line) were supplemented with fructose (control group, panel A) or glucose (intervention group, panel B) to 45 grams per day (see Research Design and Methods section). As a consequence, the minimum difference in fructose intake between both groups during the study was somewhat less than 45 grams per day. 


\section{$\underline{\text { CHAPTER } 5}$}

A
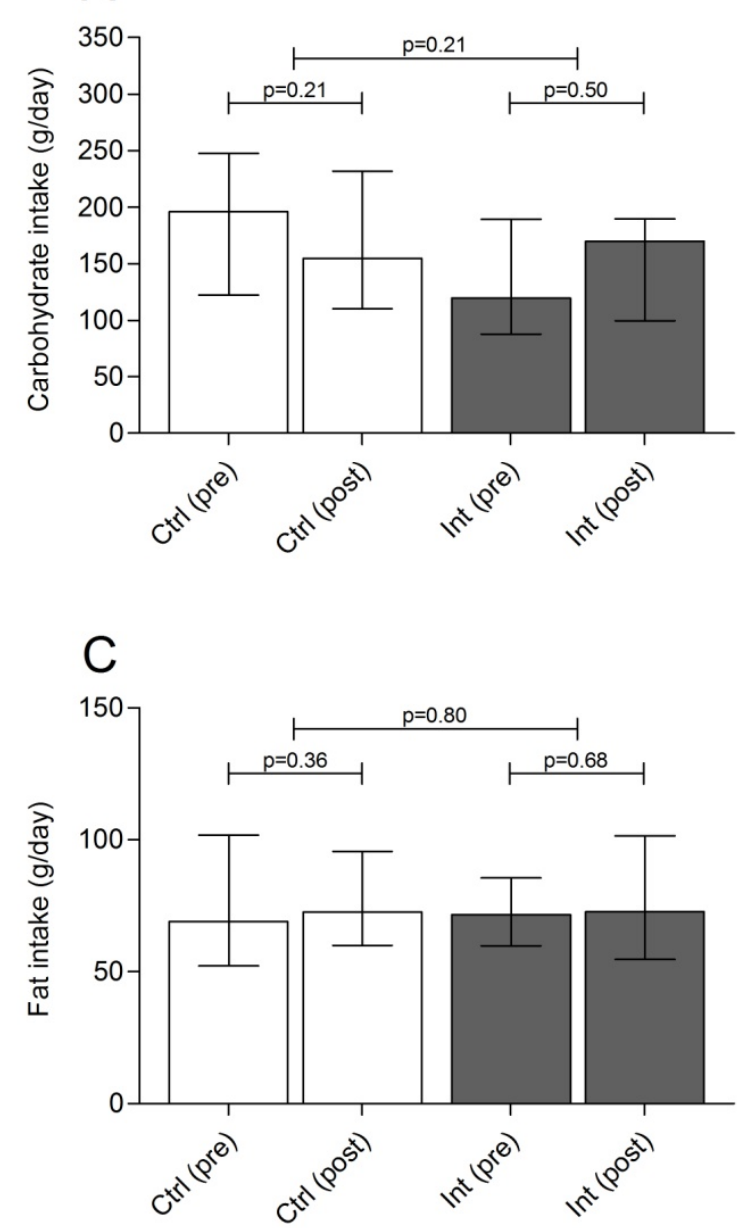

$\mathrm{B}$
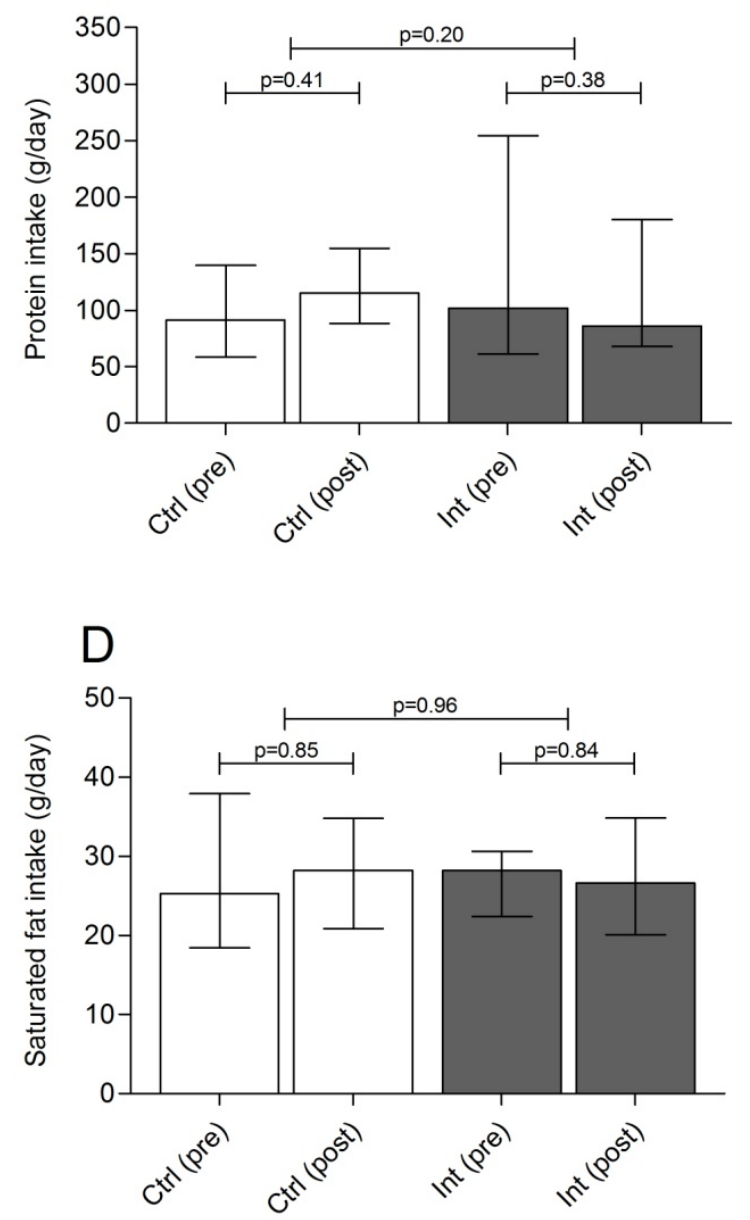

Supplementary figure 2: Macronutrient composition pre- and post-intervention. Daily carbohydrate (A), protein (B), total fat (C) and saturated fat (D) intake in the control (Ctrl; white bars, $n=21$ ) and intervention (Int; grey bars, $\mathrm{n}=16$ ) group at baseline (pre) and after completion of the study (post). Data are expressed as median \pm interquartile range. Differences within groups are analyzed with a Wilcoxon signed-rank test. Differences between groups are analyzed with a Mann Whitney U test. 
A

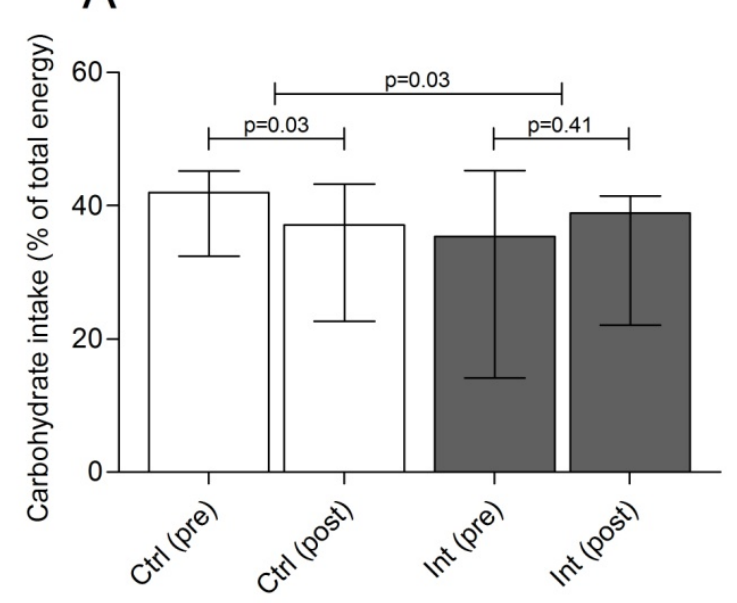

C

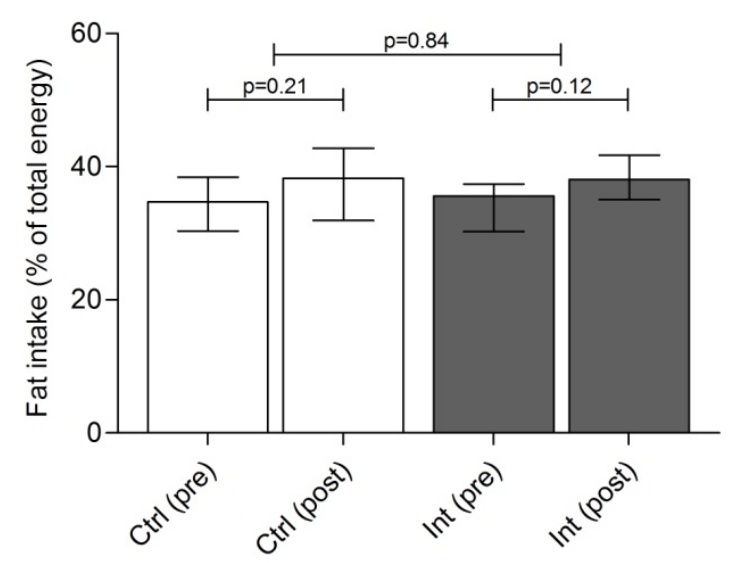

B

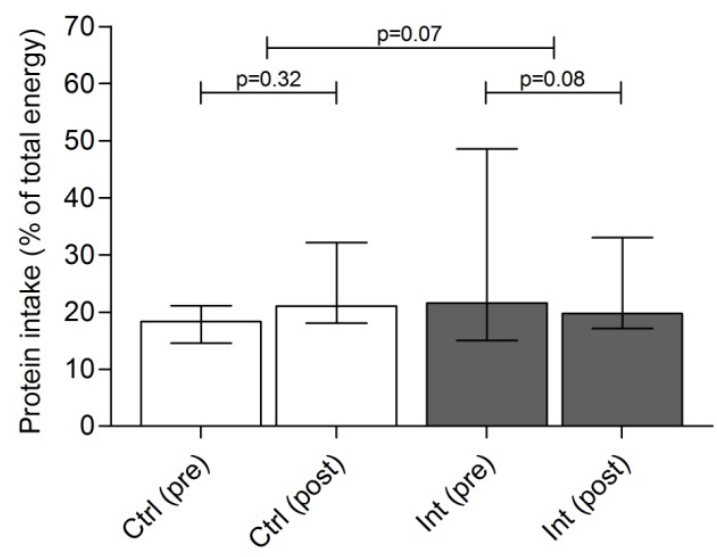

D

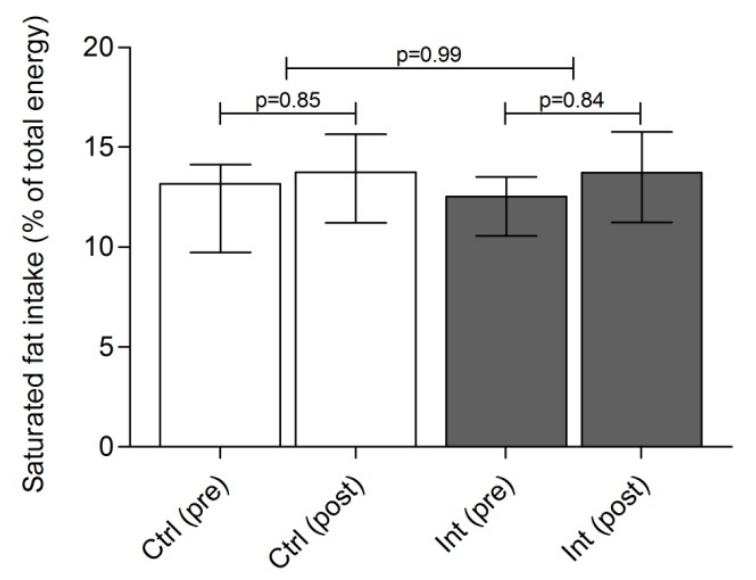

Supplementary figure 3: Macronutrient composition expressed as percentage of total energy intake pre- and post-intervention. Daily carbohydrate (A), protein (B), total fat (C) and saturated fat (D) intake expressed as percentage of daily energy intake in the control (Ctrl; white bars, $n=21$ ) and intervention (Int; grey bars, $n=16$ ) group at baseline (pre) and after completion of the study (post). Data are expressed as median \pm interquartile range. Differences within groups are analyzed with a Wilcoxon signed-rank test. Differences between groups are analyzed with a Mann Whitney U test. 


\section{$\underline{\text { CHAPTER } 5}$}

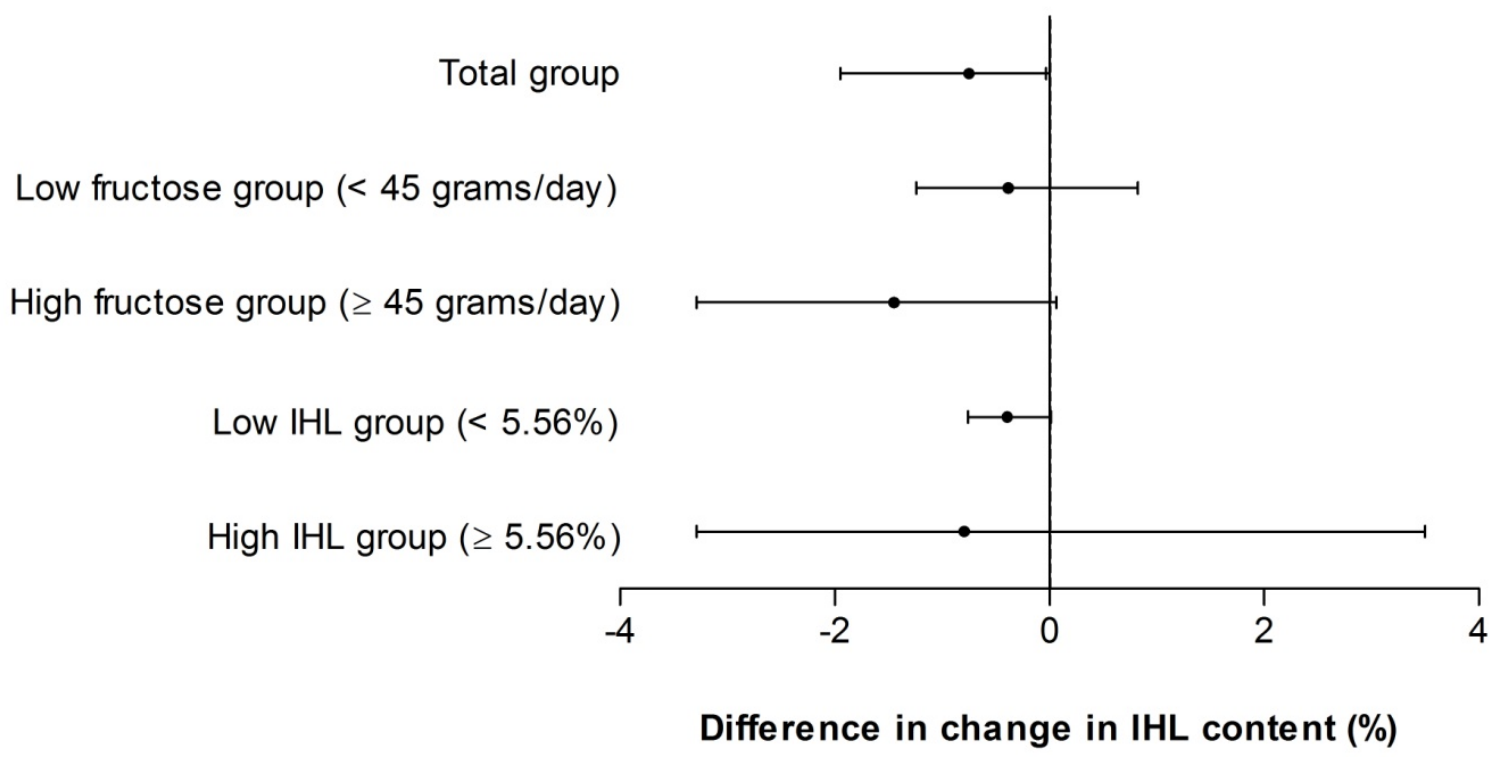

Supplementary figure 4: Subgroup analyses based on fructose intake and IHL content at baseline. Effect size of the intervention on intrahepatic lipid content stratified by fructose intake (i.e., above or below the Dutch average fructose intake, 45 grams per day) and intrahepatic lipid content (i.e., above or below the cut off value for NAFLD based on proton magnetic resonance spectroscopy, 5.56\%) at baseline. Data are expressed as median difference (with 95\%CI) between change from baseline between the control $(\mathrm{n}=21)$ and intervention $(\mathrm{n}=16)$ group. 


\section{CHAPTER 6}

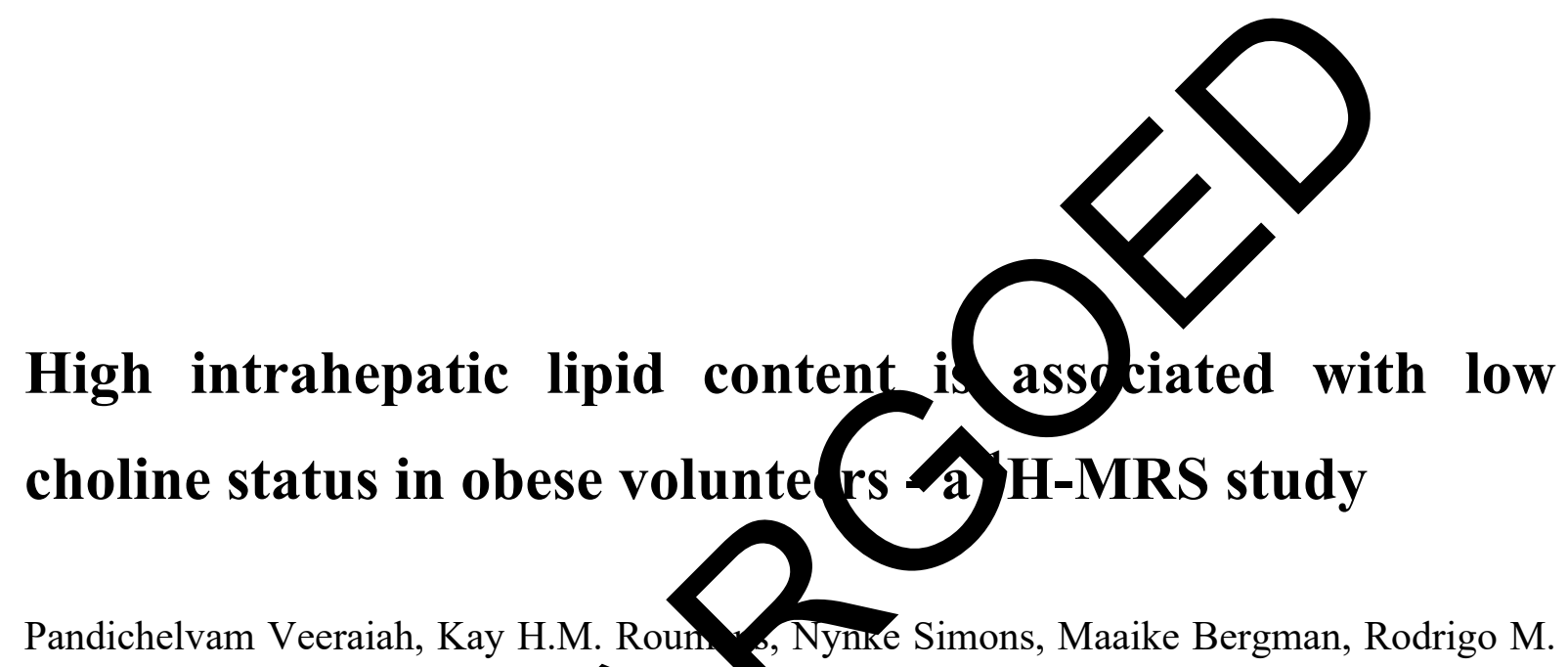

Fuentes, Matthijs K.C. Hesselink, pau Brouwers, Patrick Schrauwe $\mathrm{a}$ B. Schrauwen-Hinderling

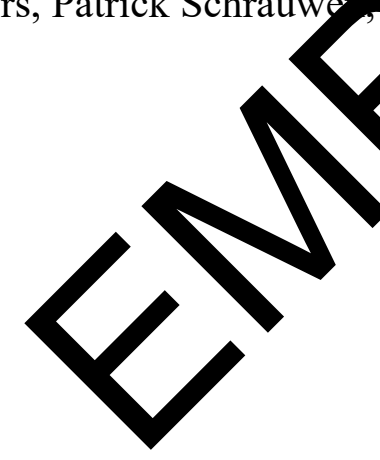




\section{CHAPTER 7}

General discussion 


\section{$\underline{\text { CHAPTER } 7}$}

\section{INTRODUCTION}

Non-alcoholic fatty liver (NAFL) has become a major threat to metabolic health. The prevalence of NAFL is increasing rapidly worldwide and is estimated to be about $25 \%$ and NAFL is predominantly observed (50-70\%) in volunteers with obesity/overweight (1). NAFL can progress into liver inflammation (non-alcoholic steatohepatitis, NASH) and severe chronic liver diseases including cirrhosis, fibrosis and hepatocellular carcinoma (HCC) (2). Currently, NAFL-related disorders such as HCC and cirrhosis are the second most common reason for liver transplantation (3). Next to being a risk factor for progression of liver disease, NAFL is also strongly associated with insulin resistance and in fact is the common risk factor for diabetes type II (4) and cardiovascular diseases (CVD) (5). Despite the known adverse health effects of NAFL, the underlying mechanism by which fat accumulates in the liver is far from understood in humans, and currently, we are mostly relying on data from animal studies. This is mainly due to the fact that tissue sampling (a biopsy) is generally needed to obtain metabolic information, and such invasive techniques are an undesirable and inappropriate method for longitudinal studies in humans. Therefore, reliable and quantitative non-invasive methodologies are warranted to study metabolic pathways involved in the development of NAFL in humans.

Over the past decade, magnetic resonance spectroscopy (MRS) has emerged as an accurate, reliable and quantitative tool to study metabolism non-invasively in humans (6) and is regularly applied in brain, heart, liver (7) and muscle (8). A major advantage of MRS is that it can be used to gain dynamic metabolic information as it is a safe technique, which does not rely on ionizing radiation. Therefore, interventional studies can use MRS repeatedly in longitudinal metabolic research to determine the response of any physiological challenge. Nowadays, ${ }^{1} \mathrm{H}-$ MRS has become the gold-standard method to measure intrahepatic lipid (IHL) content noninvasively and has been shown to correlate with histological evaluation of hepatic steatosis (9). Although MRS has been extensively used in both experimental and clinical settings, the potential of in vivo MRS has not been fully utilized yet. Investing time in optimizing MR protocols, developing new MR sequences and improving post-processing approaches can provide access to more valuable metabolic information. In this thesis, I have proposed new MRS protocols to study hepatic lipid metabolism in humans and applied these protocols successfully to show the in vivo feasibility and clinical applications in metabolic research towards understanding hepatic steatosis. 


\section{Can MRS play a role to distinguish metabolic pathways in the liver towards understanding hepatic lipid metabolism?}

The liver plays a central role in many metabolic processes. The liver regulates constant energy supply to the body through metabolism of both lipids and carbohydrates. Hepatic steatosis occurs when there is an imbalance between lipid uptake and lipid disposal. As stated in the introduction chapter 1 , several metabolic pathways contribute to the accretion of triglyceride stores in the liver: influx of circulating fatty acids, meal-derived fat retention, de novo lipogenesis (DNL), while other pathways lower the fat content of the liver, such as fatty acid oxidation (FAO) and secretion of very low-density lipoproteins (VLDL) (10). It has been shown that excessive DNL can contribute significantly to liver fat accumulation (11-13). DNL is the primary metabolic pathway by which excess dietary consumption of carbohydrates (nonlipid precursors) is being converted into fat in the liver. Although animal studies have indicated a link between DNL and NAFL, the exact relative importance of these metabolic pathways to the development of hepatic steatosis in humans is yet unknown, mainly due to the lack of reliable non-invasive methodologies. To date, the methods that measure metabolic pathways in both animal and human studies are mainly based on the use of stable-label isotopes in combination with biopsies (13-17). The work of this thesis represents an effort to develop MRS techniques that convey information about the metabolic pathways involved in fat accretion in the liver.

While ${ }^{1} \mathrm{H}-\mathrm{MRS}$ is widely used to quantify total IHL content non-invasively, this method is not able to distinguish between exogenous and endogenous lipids. Therefore, ${ }^{1} \mathrm{H}-\mathrm{MRS}$ only provides the net effect of lipid uptake, storage and oxidation (18). Exogenous dietary fat uptake is commonly measured by using fatty acid tracers, for example consumption of fatty acids that are enriched with stable isotope of ${ }^{13} \mathrm{C}$ (carbon) or ${ }^{2} \mathrm{H}$ (deuterium). The low natural abundance of ${ }^{13} \mathrm{C}(1.1 \%)$ and ${ }^{2} \mathrm{H}(0.015 \%)$ makes them ideal candidates for tracer studies as they provide a low background signal. Conventionally, ${ }^{13} \mathrm{C}$ enrichment in VLDL-TG is measured in plasma, which is considered as a surrogate marker for assessing hepatic TG pool $(14,16)$. To assess ${ }^{13} \mathrm{C}$ enrichment directly in the liver, tissue sampling is generally required which is not suitable and undesirable for human studies. Alternatively, noninvasive MR spectroscopic techniques can overcome this problem. It is known that direct ${ }^{13} \mathrm{C}$ MRS together with stable ${ }^{13} \mathrm{C}$ labeled substrates can provide an opportunity to track the low incorporation of ${ }^{13} \mathrm{C}$ labeled lipids in real time directly in the liver. This allows the quantitative assessment of contribution of exogenous dietary fat retention to total liver fat accumulation as it measures the changes in ${ }^{13} \mathrm{C}$ enrichment 
(from the baseline) noninvasively (19). However, localization of MR signal is difficult with direct ${ }^{13} \mathrm{C}$ MRS and usually the measurements are only localized by the coil and the flip angle of the excitation pulse. This can be problematic as contamination from other tissues occur, making the interpretation of data challenging. Furthermore, signal-to-noise ratio (SNR) is usually very low because of the inherently low sensitivity of direct ${ }^{13} \mathrm{C}$-MRS. Therefore, more advanced indirect ${ }^{13} \mathrm{C}$ spectral editing MR techniques have been developed to study hepatic lipid metabolism. These techniques make it possible to take advantage of the higher sensitivity of ${ }^{1} \mathrm{H}$ and better localization techniques that are established for ${ }^{1} \mathrm{H}$ and still detecting a signal that scales with ${ }^{13} \mathrm{C}$ abundance: this is achieved by observing the ${ }^{1} \mathrm{H}$ signal, however only retaining the ${ }^{1} \mathrm{H}$ signal that is coupled to ${ }^{13} \mathrm{C}$ nuclei. Such "indirect" ${ }^{13} \mathrm{C}$ MRS can be performed mainly by two ways; either by subtraction-based J-difference editing (JDE) method or by applying multiple quantum coherence-based method that allow single shot acquisitions (20). Although subtraction-based J-difference editing (JDE, e.g., POCE) has been frequently applied in brain to study glucose metabolism $(21,22)$, it is generally not suitable for application in the liver. This is due to respiratory motion in the liver which leads to imperfect subtraction due to (large) overlap of ${ }^{1} \mathrm{H}-\left[{ }^{12} \mathrm{C}\right]$ lipid signal at $1.3 \mathrm{ppm}$. Therefore, our research group has previously introduced a quantum coherence-based method (ge-HSQC) for indirect ${ }^{13} \mathrm{C}-\mathrm{MRS}$ in the liver and demonstrated the in vivo feasibility to track ${ }^{13} \mathrm{C}$-labeled lipids into the hepatic lipid pool in the liver (23). However, using the previously developed method of ge-HSQC, a ${ }^{13} \mathrm{C}$ lipid signal increase in the liver could be detected, but the sensitivity of the method is insufficient to investigate postprandial metabolism in more detail (e.g., differentiate between retention of different kinds of fatty acids or quantify the rate of DNL by quantifying the ${ }^{13} \mathrm{C}$ lipid signal resulting from ${ }^{13} \mathrm{C}$ sugar intake). Therefore, in chapter 4 , we developed a new and more sensitive approach for ${ }^{13} \mathrm{C}$ tracking applications in the human liver by improving the robustness of a regular J-difference editing (JDE) technique with use of Bllinear Rotation Decoupling (BIRD) filter. Interestingly, our proposed approach with BIRD filter not only reduced the subtraction artifact efficiently but also achieved a 2 -fold higher ${ }^{13} \mathrm{C}$ signal gain as compared to the previously applied method of ge-HSQC (thereby indirectly also increasing time resolution of the methodology). We demonstrated the in vivo feasibility of our proposed approach and successfully quantified even the low natural abundance of ${ }^{1} \mathrm{H}-\left[{ }^{13} \mathrm{C}\right]$ hepatic lipid signals in healthy volunteers. Therefore, a reliable indirect ${ }^{13} \mathrm{C}$ spectral editing MR method has been developed for ${ }^{13} \mathrm{C}$ tracking applications in the human liver. This new approach can be used to distinguish the exogenous contribution of different dietary sources into the hepatic fat pool with more sensitivity. Furthermore, this approach could bring us a step closer to the direct 
measurement of DNL in the human liver with the use of ${ }^{13} \mathrm{C}$ labeled glucose or fructose, just like it was previously performed in small animal models (24). However, it is yet unclear whether the newly developed indirect ${ }^{13} \mathrm{C}$ MR method is sensitive enough to determine rates of DNL in vivo, as this also depends on the degree to which dietary sugar is converted to lipids by DNL which will in turn determine the amount of ${ }^{13} \mathrm{C}$ labeled glucose or fructose required to track low incorporation of ${ }^{13} \mathrm{C}$ labeled hepatic lipids. Considering the high costs of ${ }^{13} \mathrm{C}$ labeled glucose or fructose, such human experiments will certainly be very expensive. To complicate things further, it has been suggested in a previous study that the majority of ${ }^{13} \mathrm{C}$ labeled sugars (38\%) are found to be oxidized as TG-glycerol fraction and only minimal amounts found in TG-fatty acids (0.5\%) (25), challenging a simple interpretation of such experiments. Considering the above-mentioned practical aspects, indirect ${ }^{13} \mathrm{C}$ MRS may not be rapidly employed on a large cohort population to measure DNL with the use of either ${ }^{13} \mathrm{C}$ labeled glucose or fructose but may rather be employed to investigate the postprandial kinetics of dietary fatty acids in more detail. Therefore, in chapter 3, we developed an alternative method to measure DNL in humans based on fatty acid profiling. Interestingly, it is known that the end product of endogenous production of fatty acids through DNL results mainly in SFAs and high rates of DNL may change hepatic lipid composition. Therefore, determining the SFA fraction directly in the liver may in theory provide an indirect measure of DNL in humans. Previous ${ }^{1} \mathrm{H}-\mathrm{MRS}$ studies have attempted to measure lipid composition using the methine (5.3 ppm) resonance, however this approach can only determine the relative amount of double bonds, as a degree of saturation ("saturation index") and cannot specifically distinguish between MUFA and PUFA content $(26,27)$. As stated in the introduction chapter 1, in principle, the distinct detection of the allylic and diallylic peak in the ${ }^{1} \mathrm{H}-\mathrm{MR}$ derived spectrum allows the estimating the MUFA and PUFA content separately, but at clinical field strength (3T), the allylic resonance is highly contaminated (overlapped) with the alpha carbonyl group, which hampers the accurate quantification of hepatic lipid composition. In chapter $\mathbf{3}$, we showed that acquiring high quality ${ }^{1} \mathrm{H}-\mathrm{MR}$ spectra with optimized water suppression and developing an extensive post-processing routine with appropriate prior knowledge, enables us to differentiate between SFA, MUFA and PUFA fractions separately in the liver. Interestingly, we showed that the hepatic SFA fraction as measured by ${ }^{1} \mathrm{H}-\mathrm{MRS}$ was positively correlated with DNL (measured by stable isotope technique, deuterated water) indicating that high rates of DNL will result in higher amounts of SFA in the liver. In line with this, we further confirmed this association between DNL and SFA fraction by showing an increased hepatic SFA fraction in GSD1a patients, a genotype which is characterized by increased rates of DNL. 


\section{$\underline{\text { CHAPTER } 7}$}

We have to be aware, that this approach measures fatty acid composition as a marker of DNL and reflects the long-term effect of DNL, as it takes time for the fat composition in the liver to change. Therefore, it is more a reflection of "habitual" DNL and acute changes in DNL cannot be visualized by this ${ }^{1} \mathrm{H}-\mathrm{MRS}$ method. Acute determination of DNL really rely on tracer studies and here, the newly developed indirect ${ }^{13} \mathrm{C}$ MRS method may be a step in the right direction.

Interestingly, plasma (VLDL)-TG composition is often used as a surrogate marker to reflect hepatic lipid composition and can therefore also be used as indicator of DNL $(13,15,17)$. Therefore, to investigate the agreement, we compared plasma VLDL-TG composition with our ${ }^{1} \mathrm{H}$ MRS based hepatic lipid composition. As expected, hepatic SFA and MUFA fractions, as determined by our newly developed ${ }^{1} \mathrm{H}$-MRS method were positively correlated with corresponding SFA and MUFAs measured in plasma VLDL-TG composition. However, interestingly, unlike the SFA fraction determined by MRS, SFA fractions in plasma VLDL-TG did not correlate with DNL measured by deuterated water. This illustrates the added value of determining fatty acid composition directly in the liver by MRS above investigating fatty acid composition in the VLDL fraction as a surrogate marker for DNL.

Taken together, MRS can certainly be instrumental in advancing our understanding of the various metabolic pathways how lipids get into the liver and concretely, this thesis has contributed to this by developing MR tools that can be used to track dietary fat and means to investigate DNL further.

\section{Can MRS characterize impaired metabolic health associated with hepatic steatosis?}

In addition to the abnormal IHL content, there are other characteristics that may be different in NAFL and can be identified non-invasively through MRS. We showed in chapter 2 that $\mathrm{T}_{2}$ relaxation times, measured in a total of 104 volunteers, differ among individuals. This is consistent with earlier MRS reports where the variation in $T_{2}$ relaxation times was reported among individuals, however the underlying reason was not yet explored in those studies $(28,29)$. Interestingly, we found that volunteers with high liver fat have shorter water $T_{2}$ and longer lipid $\mathrm{T}_{2}$ as compared to volunteers with low liver fat, indicating that $\mathrm{T}_{2}$ relaxation times are associated with level of hepatic steatosis. In line with this, a similar $\mathrm{T}_{2}$ variation was shown before in rats (30), which was suggested to be a characteristic feature for fatty liver tissue. The dependency of $T_{2}$ on the degree of steatosis is reported here for first time in humans which 
could be considered as an important characteristic feature of fatty liver that can be characterized non-invasively by ${ }^{1} \mathrm{H}-\mathrm{MRS}$.

It is yet unclear what is underlying the changes in $T_{2}$ relaxation time with increasing fat content, but it is known that increased hepatic iron load, which is more common in NAFL $(31,32)$, shortens $T_{2}$ relaxation times and may in principle be underlying the shorter $T_{2}$ relaxation times that we found with increasing degree of NAFL severity. However, if iron content was indeed different, also $\mathrm{T}_{2}$ relaxation time of lipids is expected to be shortened and in fact we see the opposite, making it unlikely that in our relatively healthy population, iron content is the underlying reason of our findings. Differences in glycogen content may be another factor to be involved in modulating $T_{2}$ relaxation of water specifically. Glycogen binds water and the bound water is known to have a shortened $\mathrm{T}_{2}$ relaxation time with respect to unbound water $(33,34)$. Therefore, the shortened $\mathrm{T}_{2}$ relaxation time may reflect increased glycogen concentrations, but this requires further study. In line with this notion, metabolically challenged patients that are prone to develop NAFL are also thought to have rather inert glycogen stores, not able to mobilize glycogen as easily as metabolically healthy people (35). Therefore, the measurement of $\mathrm{T}_{2}$ relaxation times by ${ }^{1} \mathrm{H}-\mathrm{MRS}$ is not only important to perform accurate $\mathrm{T}_{2}$ correction of MRS signals for the absolute estimation of IHL content but may also be important to characterize metabolic health associated with hepatic steatosis.

It has been suggested from animal studies that not only the total IHL content, but also the fatty acid composition, especially the saturated, mono- and poly-unsaturated fatty acids (SFA, MUFA and PUFA respectively) in the liver may be important in determining the physiological consequences of fatty liver. Animal studies have suggested that specifically saturated fatty acids play an important role in the negative metabolic effects of NAFL, such as on insulin sensitivity (36-38). Therefore, distinguishing the total IHL pool stored in the liver may help to better characterize the pathophysiology of NAFLD. Using our newly developed ${ }^{1} \mathrm{H}-\mathrm{MR}$ methodology, we showed the clinical relevance of determining hepatic lipid composition in three different metabolically challenged populations such as diabetes type II, GSD1a patients and NAFL (chapter 3). Interestingly, we found that hepatic lipid composition was altered and that specifically the SFA fraction was elevated in all three populations as compared to healthy obese controls. Moreover, we observed that specifically the SFA fraction in the liver is associated with insulin resistance. This finding supports the hypothesis that negative health effects are conveyed by SFA in the liver and showed clinical relevance of measuring hepatic 


\section{$\underline{\text { CHAPTER } 7}$}

lipid composition, specifically SFA fraction. While SFA is positively correlated with DNL, MUFA is negatively correlated with DNL indicating that upon increased DNL, the endogenous production of SFA is not necessarily accompanied by subsequent desaturation to MUFAs in humans. This is in contrast to the findings reported earlier in animal studies where desaturation was shown to be upregulated together with increase in lipogenic enzymes (39). This illustrates the unique role of MRS in increasing our understanding of hepatic lipid metabolism in truly translational research in humans. Thus, ${ }^{1} \mathrm{H}-\mathrm{MRS}$ plays a vital role to characterize the impaired metabolic health associated with NAFL and specifically the SFA fraction determined by our newly developed ${ }^{1} \mathrm{H}-\mathrm{MRS}$ is a clinically important target in NAFL.

In addition to the quantification of triglyceride (hepatic lipid and composition), other metabolites that may be involved in the etiology of NAFL are detectable by ${ }^{1} \mathrm{H}-\mathrm{MRS}$. For example, the trimethyl-ammonium (TMA) group of choline can be detected non-invasively in the ${ }^{1} \mathrm{H}-\mathrm{MR}$ derived spectrum at $3.20 \mathrm{ppm}$. Choline is an essential nutrient and involved in several biochemical pathways: serving as a neurotransmitter, methyl donor and an essential component of cellular membranes in regulating lipid metabolism (40). Although cholinedeficient diets are often used to induce NAFL in animal models to study hepatic lipid metabolism, experimental human data on hepatic choline status and its relationship with NAFL is scarce.

Since choline is generally considered as a biomarker for tumor tissue, previous ${ }^{1} \mathrm{H}-\mathrm{MRS}$ studies measured hepatic choline content mainly in tumor patients (41-44) but no data is available on combined quantification of lipid composition and choline content on healthy individuals. This is mainly due to the lack of appropriate non-invasive techniques to quantify lipid composition and it is also challenging to detect the small resonance of choline accurately. As the choline peak is visible in between the large resonances of water $(4.7 \mathrm{ppm})$ and lipids $(1.3 \mathrm{ppm})$ in the ${ }^{1} \mathrm{H}-\mathrm{MR}$ derived spectrum, very efficient water suppression and robust post-processing are warranted for the accurate detection of hepatic choline content in vivo. Since choline has a long $\mathrm{T}_{2}$ relaxation time (approximately $77 \mathrm{~ms}$ ) (45), earlier studies preferred to apply long TE MRS to detect the choline peak, minimizing contamination by lipid signals due to relative suppression of the lipid signal at long TE. However, while using a long TE, not only the lipid signal is reduced, but also a considerable $\mathrm{T}_{2}$ associated signal loss for choline will occur, which hampers accurate detection of choline peak due to low SNR. Moreover, long TE MRS where lipids are suppressed, does not provide combined information of lipid content, lipid 
composition and choline in the liver. Therefore, in chapter 6, we showed that the TMA/choline peak can be detected and can be reliably quantified next to the lipid composition by ${ }^{1} \mathrm{H}-\mathrm{MRS}$ at $3 \mathrm{~T}$, when using optimized water suppression and robust-post processing approach. This opens the way to investigate hepatic choline metabolism in more detail, and here we showed for the first time that hepatic choline content is negatively associated with increasing level of steatosis, measured in a large group of obese/overweight volunteers with a wide range of IHL content, suggesting that the choline status may be an important parameter of liver metabolic health.

Choline functions as methyl donor and is the main precursor for PC synthesis. It is known that intracellular PC synthesis and methylation status is critical for inhibiting the maturation and activation of sterol regulatory element-binding protein 1 (SREBP1), a major transcription factor that promotes lipogenesis (46-48). As stated before, the activation of lipogenesis results mainly in saturated fatty acids (39), indeed we found that the saturated fatty acids were negatively associated with increasing choline levels. However, as this relationship was not significant anymore after adjustment for total IHL, it is presently unclear whether this reflects a relationship with specifically SFA or rather total fat. Furthermore, as this data is associative, it is unknown whether there is a causal relationship and whether low choline status could increase the rates of DNL through upregulation of transcription factors (SREBP1c) that promote lipogenesis. This is however supported by few earlier reports where choline supplementation in animal models was shown to downregulate the expression of lipogenic genes (49). Therefore, future human interventional studies will have to investigate the underlying mechanism of the observed low choline status in volunteers with steatosis. Furthermore, future studies will have to address whether normalization of choline levels is beneficial to liver metabolic health and whether the observed low hepatic choline status in volunteers with severe steatosis could be considered as one of the primary metabolic abnormalities associated with NAFL. Thus, choline may play a vital role in the development of NAFL and therefore quantifying choline directly in the liver through ${ }^{1} \mathrm{H}-\mathrm{MRS}$ would provide valuable mechanistic insight towards understanding the pathophysiology of NAFL.

Taken together, these results suggest that next to the total IHL content, there are other specific characteristic features of NAFL noted in this thesis, such as reduced water $\mathrm{T}_{2}$, increased lipid $\mathrm{T}_{2}$, low choline status and high SFA fraction in the liver. Moreover, we have shown the in vivo feasibility to measure all those metabolic features of NAFL non-invasively through ${ }^{1} \mathrm{H}-\mathrm{MRS}$ 


\section{$\underline{\text { CHAPTER } 7}$}

and nicely illustrated the potential use of in vivo MRS for metabolic research in relation to hepatic steatosis.

\section{CONCLUDING REMARKS AND FUTURE PERSPECTIVES}

MRS is a well-established non-invasive tool to study metabolism in vivo and it has a vital role in metabolic research to study ectopic fat stores. MRS is extensively applied in clinical research because of its dynamic nature and is frequently used to measure total IHL content in the liver. We have however shown in the studies presented in this thesis that there is much more metabolic information accessible with MRS by applying sophisticated, specialized MR sequences and extensive post-processing approaches. We have shown the in vivo feasibility of our proposed MRS methodology in identifying metabolic pathways in the liver towards understanding hepatic lipid metabolism. Moreover, we have shown the potential role of in vivo MRS to characterize several features of impaired metabolic health associated with NAFL in humans, which is demonstrated in this thesis by showing the alterations in hepatic $\mathrm{T}_{2}$ relaxation times, fatty acid composition and choline concentrations in volunteers with steatosis.

In future studies, the observed relationship between the SFA fraction and insulin sensitivity needs to be investigated further and the same holds true for the relationship between choline and hepatic fat content. It is important to note that the evidence presented in this thesis about negative health effects of SFA fraction and beneficial influence of hepatic choline levels, are only associative. Therefore, future interventional studies with specific diets are required to show whether reducing the SFA content and increasing choline availability in the liver has beneficial effects on liver metabolic health. 


\section{REFERENCES}

1. Loomba R, Sanyal AJ. The global NAFLD epidemic. Nat Rev Gastroenterol Hepatol. 2013;10(11):686-90.

2. Calzadilla Bertot L, Adams LA. The Natural Course of Non-Alcoholic Fatty Liver Disease. Int J Mol Sci. 2016 May 20;17(5).

3. Chedid MF. Nonalcoholic Steatohepatitis: The Second Leading Indication for Liver Transplantation in the USA. Dig Dis Sci. 2017;62(10):2621-2.

4. Brouwers B, Schrauwen-Hinderling VB, Jelenik T, Gemmink A, Havekes B, Bruls Y, et al. Metabolic disturbances of non-alcoholic fatty liver resemble the alterations typical for type 2 diabetes. Clin Sci Lond Engl 1979. 2017 Aug 1;131(15):1905-17.

5. Brouwers MCGJ, Simons N, Stehouwer CDA, Isaacs A. Non-alcoholic fatty liver disease and cardiovascular disease: assessing the evidence for causality. Diabetologia. 2020;63(2):253-60.

6. van de Weijer T, Schrauwen-Hinderling VB. Application of Magnetic Resonance Spectroscopy in metabolic research. Biochim Biophys Acta Mol Basis Dis. 2019 01;1865(4):741-8.

7. Szczepaniak LS, Nurenberg P, Leonard D, Browning JD, Reingold JS, Grundy S, et al. Magnetic resonance spectroscopy to measure hepatic triglyceride content: prevalence of hepatic steatosis in the general population. Am J Physiol Endocrinol Metab. 2005 Feb;288(2):E462-468.

8. Vock P, Hoppeler H, Hartl W, Fritschy P. Combined Use of Magnetic Resonance Imaging (MRI) and Spectroscopy (MRS) by Whole Body Magnets in Studying Skeletal Muscle Morphology and Metabolism. Invest Radiol. 1985 Aug;20(5):486-91.

9. Roldan-Valadez E, Favila R, Martínez-López M, Uribe M, Ríos C, Méndez-Sánchez N. In vivo 3T spectroscopic quantification of liver fat content in nonalcoholic fatty liver disease: Correlation with biochemical method and morphometry. J Hepatol. 2010 Oct;53(4):732-7.

10. Roumans KHM, Basset Sagarminaga J, Peters HPF, Schrauwen P, Schrauwen-Hinderling VB. Liver fat storage pathways: methodologies and dietary effects. Curr Opin Lipidol. 2020 Nov 23;

11. Matikainen N, Adiels M, Söderlund S, Stennabb S, Ahola T, Hakkarainen A, et al. Hepatic lipogenesis and a marker of hepatic lipid oxidation, predict postprandial responses of triglyceride-rich lipoproteins. Obes Silver Spring Md. 2014 Aug;22(8):1854-9.

12. Gluchowski NL, Gabriel KR, Chitraju C, Bronson RT, Mejhert N, Boland S, et al. Hepatocyte Deletion of Triglyceride-Synthesis Enzyme Acyl CoA: Diacylglycerol Acyltransferase 2 Reduces Steatosis Without Increasing Inflammation or Fibrosis in Mice. Hepatol Baltim Md. 2019 Dec;70(6):1972-85.

13. Lambert JE, Ramos-Roman MA, Browning JD, Parks EJ. Increased de novo lipogenesis is a distinct characteristic of individuals with nonalcoholic fatty liver disease. Gastroenterology. 2014 Mar;146(3):72635 .

14. Barrows BR, Parks EJ. Contributions of different fatty acid sources to very low-density lipoproteintriacylglycerol in the fasted and fed states. J Clin Endocrinol Metab. 2006 Apr;91(4):1446-52.

15. Green CJ, Pramfalk C, Charlton CA, Gunn PJ, Cornfield T, Pavlides M, et al. Hepatic de novo lipogenesis is suppressed and fat oxidation is increased by omega-3 fatty acids at the expense of glucose metabolism. BMJ Open Diabetes Res Care. 2020 Mar;8(1).

16. Timlin MT, Barrows BR, Parks EJ. Increased dietary substrate delivery alters hepatic fatty acid recycling in healthy men. Diabetes. 2005 Sep;54(9):2694-701. 
17. Donnelly KL, Smith CI, Schwarzenberg SJ, Jessurun J, Boldt MD, Parks EJ. Sources of fatty acids stored in liver and secreted via lipoproteins in patients with nonalcoholic fatty liver disease. J Clin Invest. 2005 May;115(5):1343-51.

18. Lindeboom L, Nabuurs CI, Hesselink MKC, Wildberger JE, Schrauwen P, Schrauwen-Hinderling VB. Proton magnetic resonance spectroscopy reveals increased hepatic lipid content after a single high-fat meal with no additional modulation by added protein. Am J Clin Nutr. 2015 Jan;101(1):65-71.

19. Ravikumar B, Carey PE, Snaar JEM, Deelchand DK, Cook DB, Neely RDG, et al. Real-time assessment of postprandial fat storage in liver and skeletal muscle in health and type 2 diabetes. Am J Physiol Endocrinol Metab. 2005 Apr;288(4):E789-797.

20. Choi I-Y, Andronesi OC, Barker P, Bogner W, Edden RAE, Kaiser LG, et al. Spectral editing in $1 \mathrm{H}$ magnetic resonance spectroscopy: Experts' consensus recommendations. NMR Biomed. n/a(n/a):e4411.

21. de Graaf RA, Mason GF, Patel AB, Behar KL, Rothman DL. In vivo 1H-[13C]-NMR spectroscopy of cerebral metabolism. NMR Biomed. 2003 Nov;16(6-7):339-57.

22. Fitzpatrick SM, Hetherington HP, Behar KL, Shulman RG. The flux from glucose to glutamate in the rat brain in vivo as determined by $1 \mathrm{H}$-observed, 13C-edited NMR spectroscopy. J Cereb Blood Flow Metab Off J Int Soc Cereb Blood Flow Metab. 1990 Mar;10(2):170-9.

23. Lindeboom L, de Graaf RA, Nabuurs CI, van Ewijk PA, Hesselink MK, Wildberger JE, et al. Quantum coherence spectroscopy to measure dietary fat retention in the liver. JCI Insight. 2016 Aug 18;1(13):e84671.

24. Jonkers R a. M, Geraedts TR, L. J.C. Loon V, Nicolay K, Prompers JJ. Multitissue assessment of in vivo postprandial intracellular lipid partitioning in rats using localized $1 \mathrm{H}-[\mathrm{13C}]$ magnetic resonance spectroscopy. Magn Reson Med. 2012;68(4):997-1006.

25. Chong MF-F, Fielding BA, Frayn KN. Mechanisms for the acute effect of fructose on postprandial lipemia. Am J Clin Nutr. 2007 Jun;85(6):1511-20.

26. Lundbom J, Hakkarainen A, Söderlund S, Westerbacka J, Lundbom N, Taskinen M-R. Long-TE 1H MRS suggests that liver fat is more saturated than subcutaneous and visceral fat. NMR Biomed. 2011 Apr;24(3):238-45.

27. Lundbom J, Hakkarainen A, Fielding B, Söderlund S, Westerbacka J, Taskinen M-R, et al. Characterizing human adipose tissue lipids by long echo time 1H-MRS in vivo at 1.5 Tesla: validation by gas chromatography. NMR Biomed. 2010 Jun;23(5):466-72.

28. Guiu B, Petit J-M, Loffroy R, Ben Salem D, Aho S, Masson D, et al. Quantification of Liver Fat Content: Comparison of Triple-Echo Chemical Shift Gradient-Echo Imaging and in Vivo Proton MR Spectroscopy. Radiology. 2009 Jan 1;250(1):95-102.

29. Chabanova E, Bille DS, Thisted E, Holm J-C, Thomsen HS. MR spectroscopy of liver in overweight children and adolescents: Investigation of 1H T2 relaxation times at 3 T. Eur J Radiol. 2012 May 1;81(5):811-4.

30. Song K-H, Baek H-M, Lee D-W, Choe B-Y. In vivo proton magnetic resonance spectroscopy of liver metabolites in non-alcoholic fatty liver disease in rats: $\mathrm{T} 2$ relaxation times in methylene protons. Chem Phys Lipids. 2015 Oct;191:1-7.

31. Horng DE, Hernando D, Reeder SB. Quantification of liver fat in the presence of iron overload. J Magn Reson Imaging JMRI. 2017 Feb;45(2):428-39.

32. Sharma P, Altbach M, Galons J-P, Kalb B, Martin DR. Measurement of liver fat fraction and iron with MRI and MR spectroscopy techniques. Diagn Interv Radiol Ank Turk. 2014 Feb;20(1):17-26.

33. Price TB, Gore JC. Effect of muscle glycogen content on exercise-induced changes in muscle T2 times. J Appl Physiol Bethesda Md 1985. 1998 Apr;84(4):1178-84. 
34. Gore JC, Brown MS, Mizumoto CT, Armitage IM. Influence of glycogen on water proton relaxation times. Magn Reson Med. 1986 Jun;3(3):463-6.

35. Magnusson I, Rothman DL, Katz LD, Shulman RG, Shulman GI. Increased rate of gluconeogenesis in type II diabetes mellitus. A 13C nuclear magnetic resonance study. J Clin Invest. 1992 Oct;90(4):1323-7.

36. Timmers S, de Vogel-van den Bosch J, de Wit N, Schaart G, van Beurden D, Hesselink M, et al. Differential effects of saturated versus unsaturated dietary fatty acids on weight gain and myocellular lipid profiles in mice. Nutr Diabetes. 2011 Jul;1(7):e11-e11.

37. Han P, Zhang Y-Y, Lu Y, He B, Zhang W, Xia F. Effects of different free fatty acids on insulin resistance in rats. Hepatobiliary Pancreat Dis Int HBPD INT. 2008 Feb;7(1):91-6.

38. Mäkinen S, Nguyen YH, Skrobuk P, Koistinen HA. Palmitate and oleate exert differential effects on insulin signalling and glucose uptake in human skeletal muscle cells. Endocr Connect. 2017 Jul;6(5):331-9.

39. Roumans KHM, Lindeboom L, Veeraiah P, Remie CME, Phielix E, Havekes B, et al. Hepatic saturated fatty acid fraction is associated with de novo lipogenesis and hepatic insulin resistance. Nat Commun. 2020 20;11(1):1891.

40. Sherriff JL, O’Sullivan TA, Properzi C, Oddo J-L, Adams LA. Choline, Its Potential Role in Nonalcoholic Fatty Liver Disease, and the Case for Human and Bacterial Genes. Adv Nutr Bethesda Md. 2016 Jan;7(1):513.

41. Li C-W, Kuo YC, Chen C-Y, Kuo Y-T, Chiu Y-Y, She F-O, et al. Quantification of choline compounds in human hepatic tumors by proton MR spectroscopy at 3 T. Magn Reson Med. 2005 Apr;53(4):770-6.

42. Fischbach F, Schirmer T, Thormann M, Freund T, Ricke J, Bruhn H. Quantitative proton magnetic resonance spectroscopy of the normal liver and malignant hepatic lesions at 3.0 Tesla. Eur Radiol. 2008 Nov;18(11):2549-58.

43. Zhang L, Zhao X, Ouyang H, Wang S, Zhou C. Diagnostic value of 3.0T (1)H MRS with choline-containing compounds ratio $(\triangle \mathrm{CCC})$ in primary malignant hepatic tumors. Cancer Imaging Off Publ Int Cancer Imaging Soc. 2016 Aug 22;16(1):25.

44. Ter Voert EEGW, Heijmen L, van Asten JJA, Wright AJ, Nagtegaal ID, Punt CJA, et al. Levels of cholinecontaining compounds in normal liver and liver metastases of colorectal cancer as recorded by $1 \mathrm{H}$ MRS. NMR Biomed. 2019;32(1):e4035.

45. Ouwerkerk R, Pettigrew RI, Gharib AM. Liver metabolite concentrations measured with $1 \mathrm{H}$ MR spectroscopy. Radiology. 2012 Nov;265(2):565-75.

46. Walker AK, Jacobs RL, Watts JL, Rottiers V, Jiang K, Finnegan DM, et al. A conserved SREBP1/phosphatidylcholine feedback circuit regulates lipogenesis in metazoans. Cell. 2011 Nov 11;147(4):84052.

47. Yahagi N, Shimano H, Hasty AH, Amemiya-Kudo M, Okazaki H, Tamura Y, et al. A crucial role of sterol regulatory element-binding protein-1 in the regulation of lipogenic gene expression by polyunsaturated fatty acids. J Biol Chem. 1999 Dec 10;274(50):35840-4.

48. Houten SM, Argmann CA. New driver for lipid synthesis. Cell. 2011 Nov 11;147(4):719-21.

49. Jack-Roberts C, Joselit Y, Nanobashvili K, Bretter R, Malysheva OV, Caudill MA, et al. Choline Supplementation Normalizes Fetal Adiposity and Reduces Lipogenic Gene Expression in a Mouse Model of Maternal Obesity. Nutrients. 2017 Aug 18;9(8). 

APPENDICES

Summary 


\section{APPENDICES}

\section{SUMMARY}

The prevalence of non-alcoholic fatty liver (NAFL/hepatic steatosis) is increasing rapidly worldwide and predominantly observed in obese/overweight populations. NAFL can progress into liver inflammation (non-alcoholic steatohepatitis, NASH) and end stage of all chronic liver diseases including cirrhosis and hepatocellular carcinoma (HCC). Moreover, NAFL has been shown to be strongly associated with insulin resistance and is a major risk factor for the development of diabetes type II and cardiovascular diseases (CVD). Despite the known negative health effects of NAFL, understanding primary metabolic abnormalities that drive hepatic fat accretion are still far from understood in humans and mainly rely on data obtained from animal studies. This is mainly due to lack of reliable and quantitative non-invasive methodologies to study the metabolic pathways involved in NAFL in humans.

There is increasing interest in applying in vivo magnetic resonance spectroscopy (MRS) in metabolic research, as it is a safe technique, and it provides an opportunity to study metabolism non-invasively. Furthermore, a major advantage of MRS is that metabolism can be studied quantitively and dynamically. However, the potential of in vivo MRS has not been fully utilized yet. In this thesis, I have focussed on the development of new MRS protocols to study hepatic lipid metabolism in humans and successfully applied those MR protocols to show clinical applications in metabolic research focused on improving our understanding of the origins of hepatic steatosis.

${ }^{1} \mathrm{H}-\mathrm{MRS}$ is widely used to measure IHL content, and it has been well demonstrated in earlier studies that absolute estimation of IHL content is possible with ${ }^{1} \mathrm{H}-\mathrm{MRS}$. However, there are variations among different research sites as to which fat quantification formulas are used to calculate fat percentage in absolute terms. All calculations use the comparison of the lipid and water signals however, they are based on ratios of $\mathrm{CH}_{2} / \mathrm{H}_{2} \mathrm{O}, \mathrm{CH}_{2} /\left(\mathrm{CH}_{2}+\mathrm{H}_{2} \mathrm{O}\right)$, or on the sum of all lipid peaks/total MR signal. Also, the method of quantification (integration vs peak fitting) can vary and the type of MR sequence that is used (mainly STEAM or PRESS) can differ. Also, different $\mathrm{T}_{2}$ correction strategies (individual vs average $\mathrm{T}_{2}$ correction) are used. All of these differences can ultimately lead to substantial bias in the calculated IHL\%. The impact that such differences have on the determination of the IHL\% was investigated in chapter 2. This is important as differences in calculations results variations in the numerical values of fat content, while generally, the cut-off value for hepatic steatosis is set to a fixed value (5.6\%). Therefore, we reviewed all the steps that are needed to determine absolute IHL\% 
as a weight percentage (w/w) and evaluated the bias that occurs in the calculations of IHL\% when using different formulas, incorrect $\mathrm{T}_{2}$ correction and two standard MR sequences (STEAM vs PRESS). When calculating absolute IHL\% (w/w), several assumptions are made, for example in terms of typical fatty acid density and composition and liver tissue density. Interestingly, we demonstrated that simple ratio of $\mathrm{CH}_{2} / \mathrm{CH}_{2}+\mathrm{H}_{2} \mathrm{O}$ yielded very similar numerical values as to the calculation of $\mathrm{w} / \mathrm{w} \%$ and $\mathrm{CH}_{2} /\left(\mathrm{CH}_{2}+\mathrm{H}_{2} \mathrm{O}\right)$ can therefore be used as a solid way to approximate $\mathrm{w} / \mathrm{w} \%$. To have reliable reference values, we also determined $\mathrm{T}_{2}$ relaxation times in a large group of volunteers and interestingly, we observed that water $T_{2}$ is reduced and lipid $T_{2}$ is increased with increasing severity of NAFL. Therefore, with fixed $T_{2}$ correction strategy, the difference in IHL\% is overestimated when two groups differing strongly in liver fat content are compared.

Next to determining the accurate estimation of absolute IHL\%, it is important to understand the primary metabolic pathways that drive excessive hepatic fat accumulation. De novo lipogenesis (DNL) may be an important player in this. As the end products of DNL are saturated fatty acids, determining lipid composition, specifically measuring saturated fatty acid (SFA) fraction in the liver may be an indirect measure of DNL. Therefore, we developed, validated, and successfully applied a new ${ }^{1} \mathrm{H}$-MRS protocol based on post-processing approach that enabled us to determine the hepatic fractions of SFA, mono- and poly unsaturated fatty acid fractions (MUFA and PUFA) separately, which is described in chapter $\mathbf{3}$ of this thesis. Our newly developed ${ }^{1} \mathrm{H}-\mathrm{MRS}$ methodology uses appropriate prior knowledge obtained from high-resolution ${ }^{1} \mathrm{H}-\mathrm{NMR}$ data of oil phantoms, and the overlapping allylic- and alpha-carbonyl signals were dissociated accordingly to determine lipid composition. This approach was validated in five different vegetable oil phantoms by comparing the measured to the known composition. Additionally, we validated our approach in vivo by measuring the lipid composition in subcutaneous adipose tissue in eight volunteers and compared it to the analysis by mass spectrometry in corresponding biopsies. Finally, we demonstrated the in vivo feasibility and reproducibility of our developed approach in the liver of seven healthy volunteers with varying liver fat content. Interestingly, we showed that hepatic SFA fraction as measured by ${ }^{1} \mathrm{H}-\mathrm{MRS}$ was positively correlated with DNL (measured by deuterated water) confirming that high rates of DNL will result in higher amount of SFA in the liver. Thus, we developed a new ${ }^{1} \mathrm{H}-\mathrm{MRS}$ methodology to determine lipid composition, specifically measuring SFA fraction in the liver can provide an indirect measure of DNL in humans. 


\section{APPENDICES}

While the newly developed ${ }^{1} \mathrm{H}-\mathrm{MRS}$ protocol can be used as an indirect measure of the contribution of DNL to IHL over a longer period of time, it is not possible to acutely determine DNL, for example from a meal. Such measurements are usually performed by using stable isotope tracers and in principle the conversion of ${ }^{13} \mathrm{C}$ labeled glucose could be monitored by determining ${ }^{13} \mathrm{C}$ lipid signals in the liver. Although our previously developed indirect ${ }^{13} \mathrm{C}$ MRS (ge-HSQC, based on quantum-coherence) can in principle be monitor the abundance of ${ }^{13} \mathrm{C}$ labeled hepatic lipids, this method has insufficient sensitivity for such experiments. Therefore, in chapter 4, we optimized a J-difference editing (JDE) method, which inherently yields a twofold higher signal as compared to the previously applied method of geHSQC. With the application of BIlinear Rotation Decoupling (BIRD) filter the subtraction artifact that hampers the application of such sequences in the liver was efficiently suppressed and the small signal of natural abundance $(1.1 \%)$ of ${ }^{13} \mathrm{C}$ lipid signal could be detected, even at low fat contents. However, even though sensitivity was strongly improved, it is yet unclear whether DNL can be directly determined in humans using indirect ${ }^{13} \mathrm{C}$ MRS.

In stimulating DNL, fructose has been suggested to play an especially detrimental role in liver metabolic health. While there is growing evidence from animal studies on the prominent role of fructose in IHL accumulation, such experimental human data is very limited. Therefore, in chapter 5, we investigated the effects of fructose restriction on IHL content in obese/ overweight volunteers in a double-blind randomized study. Indeed, six week of fructose restriction leads to a small but significant reduction in IHL content, indicating a beneficial influence of fructose restriction on NAFL.

Next to lipids and water, also other metabolites, such as choline have resonances in the ${ }^{1} \mathrm{H}-\mathrm{MR}$ spectrum. Choline is an interesting metabolite, as choline-deficient diets are commonly used to induce NAFL in animal models, suggesting that disturbances in choline metabolism may promote the development of NAFL. However, the choline availability in the liver of NAFL population has not yet been explored in humans. The reason is that for most ${ }^{1} \mathrm{H}-\mathrm{MRS}$ investigations, protocols are optimized for lipid determination and generally no attention is paid to the small choline resonances, which are often affected by water suppression. Also, as choline concentrations are low, long acquisition times and dedicated post-processing and fitting is important in order to quantify the choline peaks in question. In chapter 6, we were able to quantify the hepatic choline content accurately in addition to lipid composition by applying our newly developed ${ }^{1} \mathrm{H}-\mathrm{MRS}$ post-processing tool in existing ${ }^{1} \mathrm{H}-\mathrm{MRS}$ data of obese/overweight 
volunteers. Interestingly, we found that low choline status is associated with high IHL content, which may point at an involvement of choline metabolism in the development of NAFL in humans. Future interventional studies will have to investigate whether normalization of choline levels is beneficial to liver metabolic health.

In conclusion, we have shown that new MR protocols yield valuable information on metabolic pathways in humans. In addition, we have shown the applicability of our newly developed MRS protocols in humans to perform metabolic research towards understanding hepatic steatosis. 

Impact of the thesis 


\section{APPENDICES}

\section{IMPACT}

The liver plays a central role in maintaining metabolic homeostasis by regulating constant energy supply to the body through metabolism of nutrients (carbohydrates and lipids). When excess amount of fat builds up in the liver, this increases the risk for developing severe chronic liver diseases and metabolic complications including diabetes type II (T2DM) and cardiovascular diseases (CVD). Quite strikingly, 20 to $30 \%$ of the general population in Western countries have excessive storage of fat in the liver (in absence of high alcohol intake), a condition referred to as non-alcoholic fatty liver (NAFL)(1). As the obesity epidemic increases, the prevalence of NAFL increases worldwide (2). To date, our understanding of the etiology of NAFL is still limited, due to the lack of appropriate non-invasive techniques that would help us to understand the mechanisms/metabolic pathways leading to NAFL.

Magnetic Resonance Spectroscopy (MRS) provides biochemical information on cellular components and is considered a powerful tool to study metabolism in vivo in a non-invasive manner. Due to its safety (it does not rely on ionizing radiation) and due to the fact that dynamic information can be gained, MRS is extensively applied in experimental research settings to study hepatic lipid metabolism (3-6), however, the potential of in vivo MRS is not yet fully utilized. In this thesis, we have shown the potential of MRS in detecting metabolic pathways (i.e., DNL) in the liver as well as to characterize the metabolic abnormalities associated with hepatic steatosis. The interpretation of the MR derived intrahepatic lipid (IHL) \% is discussed in detail in chapter 2 and recommendations are put forward on the usage of different fat quantification formulas, to express IHL\%. We advocate to express the data in a more standardized way that is directly comparable to weight/ weight \%. The results described in chapter 2 will be of great interest to a broad public including hepatologists and clinicians to better understand the exact meaning of intrahepatic lipid (IHL) \%. Currently, various ways of expressing IHL are used, and it is often unclear how it is derived from the MRS signals of fat and water. This information will eventually help us (especially for clinicians) to define NAFL with known cut-off value of 5.6\% and to compare data from different research sites. In addition, the observed dependency of $\mathrm{T}_{2}$ relaxation times on the level of steatosis is important to be taken into account and therefore, it will help us to better characterize the pathophysiology of NAFLD in humans.

The innovative, non-invasive MRS protocols developed in the $\mathrm{PhD}$ project will be very useful overall to increase our understanding of hepatic lipid metabolism in humans, especially the 
metabolic pathways (e.g., DNL) associated with NAFL. In chapter 3, we have shown for first time that not only total IHL content, but also hepatic lipid composition, specifically the SFA fraction can be a novel target for NAFL as the SFA fraction seems to be especially strongly related to metabolic disease in humans. We have shown that the quantification of hepatic lipid composition is feasible with our newly developed post-processing tool using the same hepatic lipid spectra as acquired usually for IHL measurement. Therefore, future interventional studies with ${ }^{1} \mathrm{H}-\mathrm{MRS}$ can always quantify hepatic lipid composition (without any extra requirements) next to total IHL content. Indeed, the developed MRS protocols shown in chapter3, is already applied longitudinally in different on-going clinical studies at our department to study the efficacy of different interventions (diet, therapeutic drugs) or to test a physiological challenge (e.g., exercise, fructose) to target the metabolic pathways of DNL towards aiming treatment strategies for NAFL.

Moreover, the developed MRS protocols in this $\mathrm{PhD}$ project will also be interesting for pharmaceutical companies, as it provides an opportunity to test and develop new drugs in targeting SFA specifically for therapeutic purposes. Therefore, I anticipate that funding opportunities from industry will increase, who aim to implement new MR tools in clinical trials. Although our aim is to apply new MRS techniques to get more insight into hepatic lipid metabolism, the application of these MR techniques will not be limited to this particular topic and/or specific tissue. For example, the developed MRS protocol in chapter $\mathbf{3}$ is not only used to measure hepatic lipid composition, but it could also be applied in different tissues such as adipose tissue and visceral fat or potentially also muscle. Indeed, we already demonstrated the in vivo feasibility of our newly developed ${ }^{1} \mathrm{H}-\mathrm{MRS}$ tool to measure adipose tissue lipid composition and showed high correlation with biopsies, for a validation purpose. Moreover, it is known that PUFA content is implicated in breast cancer patients $(7,8)$, so future studies may want to investigate whether our newly developed ${ }^{1} \mathrm{H}-\mathrm{MRS}$ protocol might be applied in breast cancer patients to estimate PUFA content. Moreover, lipid composition have been implicated in regulating normal brain function $(9,10)$ and cardiovascular diseases $(11)$. Interestingly, our developed ${ }^{1} \mathrm{H}-\mathrm{MRS}$ protocol can be applied in large cohort populations to determine hepatic lipid composition as it does not require any extra cost and modifications in the implementation process.

In addition, the newly developed indirect ${ }^{13} \mathrm{C}$ MRS (described in chapter 4) can provide valuable mechanistic insight towards understanding hepatic metabolism in more detail by 


\section{APPENDICES}

identifying the contribution of different metabolic pathways in the development of NAFL. Although this method is mainly developed to track ${ }^{13} \mathrm{C}$ labeled hepatic lipids, it can be used for other ${ }^{13} \mathrm{C}$ tracking applications. For instance, the food industries can benefit from the development of such MR tools as it provides an opportunity to study metabolism noninvasively that allows us to track specific ${ }^{13} \mathrm{C}$ incorporation of labeled target metabolites from the exogenous consumption of different ${ }^{13} \mathrm{C}$ labeled substrates. Moreover, the newly developed indirect ${ }^{13} \mathrm{C}$ MRS could be used to measure metabolic fluxes, therefore the exact contribution of different metabolic pathways can be estimated in the development of NAFL. For e.g., Future studies can use our newly developed indirect ${ }^{13} \mathrm{C}$ MR method to evaluate the dietary contribution of different sugar molecules (glucose or fructose) or specific fatty acids in the development of NAFL. While the proposed ${ }^{1} \mathrm{H}-\mathrm{MRS}$ methodology to determine lipid composition is straightforward and easy to implement in any research site, the use of indirect ${ }^{13} \mathrm{C}$ MRS will be quite challenging. This is mainly due to the fact that requirement of an additional software and hardware (such as ${ }^{1} \mathrm{H} /{ }^{13} \mathrm{C}$ double tuned surface coil), use of expensive ${ }^{13} \mathrm{C}$ labeled substrates, sophisticated spectral editing MR sequence and extensive postprocessing approach. However, efforts in developing such new MR protocols and improvements in MRS methodologies will increase the potential role of in vivo MRS in metabolic research. This is illustrated in this thesis by showing that the ${ }^{1} \mathrm{H}-\mathrm{MRS}$ methodology can be employed to determine hepatic lipid composition and improved sensitivity of our indirect ${ }^{13} \mathrm{C}$-MRS can be used for ${ }^{13} \mathrm{C}$ tracking applications in the liver. Furthermore, the developed MRS protocols at clinical field strength (3T) (rather than using high field MR scanner) in the projects of my $\mathrm{PhD}$ thesis will allow us to apply the new protocols directly in many research sites without any major restrictions, as $3 \mathrm{~T}$ is commonly present in many hospitals and research institutions.

Besides developing new MR tools, the application of in vivo MRS in human interventional research is also shown in chapter 5 by performing a randomized control trail to evaluate the effects of fructose restriction in liver steatosis. The observed beneficial effect of fructose restriction in lowering IHL content can be explored in more detail in future studies by measuring hepatic lipid composition, specifically targeting DNL and to check whether SFA is altered with fructose restriction. If indeed fructose is shown to induce DNL in humans (as is the case in animal models), this is highly relevant to a broad public as the high consumption of sugar sweetened beverages in our daily life may play a significant role in the development of NAFL. 
We observed a negative relationship between choline and IHL content in chapter 6, indicating a low choline status in the overweight/ obese population, which is reported for first time in this thesis. This may suggest that correcting such low choline availability may beneficially influence metabolic health. I hope this finding will increase the interest among researchers and hepatologist to explore choline metabolism in more detail and investigate potential treatment strategies for NAFL by targeting choline status. In addition, altered choline metabolism has been implicated in several neurological disorders and tumours in different organs including brain (12), liver $(13,14)$ and breast $(15)$, although in cancer, choline tissue content is often elevated (12,14-17). It would be interesting to investigate whether our developed postprocessing approach can be applied for MRS in breast in future studies to quantify choline content in breast cancer patients.

We expect that the role of MRS will continue to grow in metabolic research. The MR protocols described in this thesis were directly applied to humans in showing clinical relevance and we believe that the proposed MR methodology will create a paradigm for future clinical applications in the human liver, to uncover metabolic information that is currently invisible.

In addition, the results and knowledge obtained from the studies performed in this thesis had been presented in several national and international scientific conferences via oral or poster presentations. This not only helped me to improve my communication and presentation skills but also improved the awareness among scientific community to realize the potential role of MRS in metabolic research. Finally, the results presented in the current thesis are or will be published as original scientific research articles with open access in peer reviewed journals, which will be assessed by scientists worldwide. This will enhance visibility of our research group as well as sharing the advances in the MRS field in translational metabolic research among the scientific community. Thus, the knowledge obtained through this research can directly be used/shared with other researchers worldwide and the MRS protocols developed in this $\mathrm{PhD}$ thesis can also be applied in multiple future research studies within the department or in making national/ international collaboration with other institutes. Last but not least, the experience and knowledge that I gained through my $\mathrm{PhD}$ will provide potential opportunities for me to achieve major personal grants in the near future and to improve myself towards fulfilling my personal scientific goals in the path of becoming an independent successful MR researcher. 


\section{REFERENCES}

1. Loomba R, Sanyal AJ. The global NAFLD epidemic. Nat Rev Gastroenterol Hepatol. 2013;10(11):686-90.

2. Younossi ZM, Koenig AB, Abdelatif D, Fazel Y, Henry L, Wymer M. Global epidemiology of nonalcoholic fatty liver disease-Meta-analytic assessment of prevalence, incidence, and outcomes. Hepatol Baltim Md. 2016 Jul;64(1):73-84.

3. Szczepaniak LS, Nurenberg P, Leonard D, Browning JD, Reingold JS, Grundy S, et al. Magnetic resonance spectroscopy to measure hepatic triglyceride content: prevalence of hepatic steatosis in the general population. Am J Physiol Endocrinol Metab. 2005 Feb;288(2):E462-468.

4. Springer F, Machann J, Claussen CD, Schick F, Schwenzer NF. Liver fat content determined by magnetic resonance imaging and spectroscopy. World J Gastroenterol. 2010 Apr 7;16(13):1560-6.

5. Longo R, Pollesello P, Ricci C, Masutti F, Kvam BJ, Bercich L, et al. Proton MR spectroscopy in quantitative in vivo determination of fat content in human liver steatosis. J Magn Reson Imaging JMRI. 1995 Jun;5(3):281-5.

6. Thomsen C, Becker U, Winkler K, Christoffersen P, Jensen M, Henriksen O. Quantification of liver fat using magnetic resonance spectroscopy. Magn Reson Imaging. 1994;12(3):487-95.

7. Bartsch H, Nair J, Owen RW. Dietary polyunsaturated fatty acids and cancers of the breast and colorectum: emerging evidence for their role as risk modifiers. Carcinogenesis. 1999 Dec;20(12):2209-18.

8. Azrad M, Turgeon C, Demark-Wahnefried W. Current evidence linking polyunsaturated Fatty acids with cancer risk and progression. Front Oncol. 2013 Sep 4;3:224.

9. Kaplan RJ, Greenwood CE. Dietary saturated fatty acids and brain function. Neurochem Res. 1998 May;23(5):615-26.

10. Bazinet RP, Layé S. Polyunsaturated fatty acids and their metabolites in brain function and disease. Nat Rev Neurosci. 2014 Dec;15(12):771-85.

11. Briggs MA, Petersen KS, Kris-Etherton PM. Saturated Fatty Acids and Cardiovascular Disease: Replacements for Saturated Fat to Reduce Cardiovascular Risk. Healthcare [Internet]. 2017 Jun 21 [cited 2021 Jan 19];5(2). Available from: https://www.ncbi.nlm.nih.gov/pmc/articles/PMC5492032/

12. Huang BY, Kwock L, Castillo M, Smith JK. Association of choline levels and tumor perfusion in brain metastases assessed with proton MR spectroscopy and dynamic susceptibility contrast-enhanced perfusion weighted MRI. Technol Cancer Res Treat. 2010 Aug;9(4):327-37.

13. Li C-W, Kuo YC, Chen C-Y, Kuo Y-T, Chiu Y-Y, She F-O, et al. Quantification of choline compounds in human hepatic tumors by proton MR spectroscopy at 3 T. Magn Reson Med. 2005 Apr;53(4):770-6.

14. Ter Voert EEGW, Heijmen L, van Asten JJA, Wright AJ, Nagtegaal ID, Punt CJA, et al. Levels of cholinecontaining compounds in normal liver and liver metastases of colorectal cancer as recorded by $1 \mathrm{H}$ MRS. NMR Biomed. 2019 Jan;32(1):e4035.

15. Chen J-H, Mehta RS, Baek H-M, Nie K, Liu H, Lin MQ, et al. Clinical characteristics and biomarkers of breast cancer associated with choline concentration measured by 1H MRS. NMR Biomed. 2011 Apr;24(3):316-24.

16. Kobus T, Wright AJ, Weiland E, Heerschap A, Scheenen TWJ. Metabolite ratios in 1H MR spectroscopic imaging of the prostate. Magn Reson Med. 2015;73(1):1-12.

17. Glunde K, Bhujwalla ZM, Ronen SM. Choline metabolism in malignant transformation. Nat Rev Cancer. 2011 Dec;11(12):835-48. 
Acknowledgements 


\section{APPENDICES}

\section{ACKNOWLEDGEMENTS}

It's time to thank all the people who immensely contributed to the thesis as well as my academic growth and who made my journey not only possible but also enjoyable for the last 4.5 years.

First, I would like to thank my promoters team Dr. Vera B. Schrauwen Hinderling, Prof. dr. Joachim E. Wildberger and Dr. Lucas Lindeboom for their continued kind support and proper guidance throughout my PhD trajectory. Indeed, it's my pleasure to have you as (co) promoters.

Dear Dr. Vera B. Schrauwen Hinderling, I learnt a lot about the applications of MRS in metabolic research and improved my research skills by working closely with you. I especially admire your patience, positive attitude, enthusiasm, and the overall freedom that you gave me to perform research independently as well as in the team. I really appreciate your insightful feedback on projects and overall inputs for the writing process that always pushed me to think critically and took my work to a higher level. Thank you for trusting me with this $\mathrm{PhD}$ project and giving me the chance (even after $1^{\text {st }} \mathrm{PhD}$ evaluation) to work in your productive research group. Also thank you very much for your continuous support in grant applications and I appreciate your help to find my career path.

Dear Prof. dr. Joachim E. Wildberger, thank you for providing me an opportunity to work in this well-established research institute and for access to excellent research facilities including MR scanners. I truly appreciate your time, encouragement, proper guidance, and confidence that you showed to help me become a successful researcher. Thank you for the useful feedback and all the opportunities to develop my research career.

Dear Dr. Lucas Lindeboom, your MR expertise is invaluable, and I gradually learnt about the technical part of MRS through our regular discussions in meetings and during MR acquisitions. Thank you for taking all the time and effort that was necessary to teach me the different MR sequences, MATLAB skills and inspiring my interest in the development of MR technologies. I am extremely thankful for your continuous support and valuable feedback on my thesis, abstracts, and manuscripts even after you left from the University.

Next, I thank the members of the assessment committee Prof. dr. Ronald P. Mensink, Prof. dr. med. Michael Roden, Dr. ir. Tom Scheenen, Prof. dr. Daisy M.A.E. Jonkers and Dr. 
Jacobus F.A. Jansen for investing their time to read and approve my thesis and/or being present at the official PhD defense.

My sincere thanks to Prof. dr. Patrick Schrauwen who was involved in all projects since the beginning of my $\mathrm{PhD}$. I highly appreciate your critical feedback in formulating research questions and methodology, and discussions (also through DMRG meetings) that impressed me in a very positive way and motivated me to learn more about human physiology.

Further, I would like to thank all members in the MR spectroscopy research team within our research (DMRG) group who worked so hard as a team in different projects and helped each other to collect MRS data, which I utilized partially to create $T_{2}$ and choline story of the thesis. Kay, you are practically my research partner and we have worked in the same project (LICO) since the beginning of my $\mathrm{PhD}$ and given presentations together for TKI (Unilever) updates. I appreciate your time and efforts in coordinating such a nice clinical study with all collaborators from abroad. I gratefully acknowledge your contribution in the choline and $\mathrm{T}_{2}$ project for collecting extensive MRS data from different populations. Also, thank you for being the paranymph in my $\mathrm{PhD}$ defense. Good luck with the completion of your thesis! Kim, thank you for your time, kind support and assistance during MR acquisitions. I really appreciate your help for acquiring extra scans in the volunteers to determine $T_{2}$ and your contribution in the indirect ${ }^{13} \mathrm{C}$ project for liver measurements. I liked the discussions about MR basics that we often had at the MR scanner room that stimulated me to think beyond my project and motivated me to learn more about MR sequences. Yvonne, you were my first officemate when I joined and helped me to settle here, thank you for showing me how to perform muscle MRS scan for HIIT study. I was happy to be a part of your carnitine project in analyzing MRS data and gratefully acknowledge your contribution in the $\mathrm{T}_{2}$ project. Also, thank you for arranging DMRG farewell party and being a strong support as paranymph in my PhD defense. Julian, you were my office mate for the last 2 years, we had nice conversations and I liked your company during MR conferences. Thank you for your time and kind support for helping me understand the MATLAB scripts. I appreciate all your help that you provided so far, and I acknowledge your contribution in the $\mathrm{T}_{2}$ project. Maaike and Rodrigo, it was nice to work with you guys on the HIIT project since it provided me a different experience of applying MRS on muscle. I enjoyed the conversations while working with both of you at MR scanners late evenings. Thank you for your contribution in the choline project and for your help in recruiting volunteers for liver MR scans. 


\section{$\underline{\text { APPENDICES }}$}

I would like to extend my gratitude to all the collaborators Prof. dr. Martijn C.G.J. Brouwers, Prof. dr. Matthijs K.C. Hesselink, Prof. dr. Jogchum Plat and Dr. Sabine Baumgartner who gave me a chance to contribute to their fascinating projects and valuable discussions during meetings that helped to obtain more insights about the application of MRS in different clinical studies.

Dear Nynke, the application of MRS in the FRUITLESS study brought us to work together and I enjoyed the time while working with you. I really appreciate the time, continuous effort, and medical expertise that you have invested in this clinical study which finally led this work to be recognized as a good publication. Also, I gratefully acknowledge your contribution in the choline and $\mathrm{T}_{2}$ project and thank you for your inputs in explaining statistics. Apart from work, during the last 4 years, we've become friends, enjoyed good times and I hope we continue meeting with families.

Dear Pomme, it's a pleasure to work with you on EFSDP project and it eventually helped me to gain more insights about lipid metabolism in different population of genetic disorders. I appreciate the continuous effort that you put on your recent publication, and I am happy to be a part of it. Thank you for your time, motivation, kind support and nice conversations.

I would like to thank all the current and ex-colleagues from the DMRG, Joris, Esther P, Tineke, Mijke, Bas, Anne, Nynke, Lotte, Vera W, Yvo, Froukje, Frieder, Charlotte, Jeremy, Sten, Edmundo, Carlijn, Anna, Stephanie, Daniel, Jakob and to everyone else for creating a healthy, friendly environment to work and stimulating discussions during our regular DMRG meetings. Thank you, Gert, Esther K, and Johanna, for processing and analyzing all the samples for the LICO and choline project. Thank you Loek and Harry for helping me to install software and solve computer related problems.

Of course, I also want to thank all the current and ex-members in the Radiology and Nuclear Medicine department, Eline, Mueez, Mohamed, Lidewij, Yentl, Rob, Rik, Genevieve, Ellen, and everyone for their friendly nature and nice conversations during MR conferences. Thank you, Roland and all MRI technicians and scientific staff who helped in planning of MR scans and gave assistance during MR acquisitions. Thank you, Estelle, for taking the time and effort to proofread the $\mathrm{T}_{2}$ manuscript. 
My research would have been impossible without the dedicated contribution of all the participants who decided to be a part of our studies. Thank you for your patience, support, and endurance, also at times when repeating MR scans while testing new MR sequences/ protocols.

I would like to thank my previous mentors Dr. Anant B. Patel and Prof. dr. N. Jon Shah who gave me a chance to work in their research group and sparked my interest in the field of magnetic resonance.

I would like to take this opportunity to thank all my teachers (especially Gunasekaran from my hometown) who supported and motivated me in the path of becoming a highly qualified researcher.

Dear Dientje and Guy, you are like one of my family members and I always remember your kind hospitality and thanks for taking good care of my family at all times which helped me to focus on my research. Thank you for the everlasting kindness and generous support which I truly admire. I am grateful for everything that you did so far to make us feel at home. Hope to see both of you soon!

Willeke, almost 5 years ago we met, and we've become good friends. Thank you for your kind help, moral support, and friendly conversations. I hope we continue meeting with our families.

Deni and Hugo, you helped me initially to settle here and supported my family. Thank you for your friendship and nice conversations. Hope we continue meeting and having dinner together.

Thank you Bhagyashree, Vinidhra, Keerthana, Pasha, Ravi, Maria, Gabriela, Annaleen and all to my friends who always provided me moral support as well as happy distractions to rest my mind outside of my research. To all other colleagues and friends who I did not name, it was great getting to know you, working, and having pleasant conversations with you. Thank you for that.

Last but not the least, I want to thank my family from my hometown in India who continuously supported me emotionally as well as spiritually and had sympathetic ears at all difficult times. 


\section{APPENDICES}

என்னை ஈன்றெடுத்த பொழுதிலிருந்து இன்றும் என்றும் எனக்கு எல்லாவிதத்திலும் உறுதுனையாக இருக்கும் எனது பெற்றோர், உடன்பிறப்பு மற்றும் குடும்பத்தினர் (வீரையா, சந்தனவள்ளி, பாலா, செல்வி, மச்சராஜா...) அனைவருக்கும் மனமார்ந்த நன்றி.

My little princess Prathista (naughty girl), you made me and Priya proud parents. It's so special and memorable that you were born in the hospital where I did my work during PhD. I am always delighted and relieved of all my stress being with you and looking at the cute smile on your face. No words are enough to describe how much I love you. Thank you!

Priya (my lovable wife), you were always there for me to motivate and provide a wise counsel towards achieving my goals. I could not have completed this thesis without your help and continuous kind support. I am lucky to have you as my life partner. Thank you for your everlasting love, care and for everything that you shared with me which has enormously helped me not only to become a successful person but also give happiness to our family. Also, thank you for your suggestions for designing the cover of this thesis. 
Scientific output 


\section{This thesis}

\section{List of Publications}

1. Veeraiah P, Brouwers K, Wildberger JE, Schrauwen-Hinderling VB, Lindeboom L. Application of a BIlinear Rotation Decoupling (BIRD) filter in combination with $J$ difference editing for indirect ${ }^{13} \mathrm{C}$ measurements in the human liver. Magn Reson Med. 2020 Dec; 84(6):2911-2917.

2. Roumans $\mathrm{KHM}^{\#}$, Lindeboom L ${ }^{\#}$, Veeraiah P, Remie CME, Phielix E, Havekes B, Bruls YMH, Brouwers MCGJ, Alssema M, Peters HPF, de Mutsert R, Staels B, Taskinen MR, Borén J, Schrauwen P, Schrauwen-Hinderling VB. Hepatic saturated fatty acid fraction determined by ${ }^{1} H-M R S$ is associated with denovo lipogenesis and hepatic insulin resistance in humans. "Equal contribution. Nat Commun. 2020; 11(1):1891, " equal contribution.

3. Simons N, Veeraiah P, Simons PIHG, Schaper NC, Kooi ME, Schrauwen-Hinderling VB, Feskens EJM, Van der Ploeg LEMC, Van den Eynde MDG, Schalkwijk CG, Stehouwer CDA, Brouwers MCGJ. Effects of fructose restriction on liver steatosis (FRUITLESS); a double-blind randomized controlled trial. Am J of Clin Nutr. 2021 Feb 2;113(2):391-400.

4. Veeraiah P, Roumans KHM, Simons N, Brouwers MCGJ, Bruls YMH, Hesselink MKC, Wildberger JE, Schrauwen P, Lindeboom L, Schrauwen-Hinderling VB. Hepatic water and lipid $T_{2}$ are associated with level of steatosis-implications in absolute fat quantification (Under review in Radiology).

5. Veeraiah P, Roumans KHM, Simons N, Bergman M, Fuentes RM, Hesselink MKC, Wildberger JE, Lindeboom L, Brouwers MCGJ, Schrauwen P, Schrauwen-Hinderling VB. High Intrahepatic lipid content is associated with low choline status in humans- a ${ }^{1} H$-MRS study at 3 Tesla (Submitted).

\section{Collaboration work during PhD}

6. Simons PIHG, Valkenburg O, Telgenkamp I, Van der Waaij KM, De Groot DM, Veeraiah P, Bons JAP, Taskinen MR, Bóren J, Schrauwen P, Rutten JHW, Derks TGJ, Cassiman D, Schalkwijk CG, Stehouwer CDA, Schrauwen-Hinderling VB, Hodson L, Brouwers MCGJ. Relationship between de novo lipogenesis and serum sex hormonebinding globulin in humans. Clin Endocrinol (Oxf).2021 Mar 14, (ahead of print).

7. Bruls Y MH, Op den Kamp YJM, Veeraiah P, Phielix E, Havekes B, Schaart G, Kornips E, Wildberger JE, Hesselink MK, Schrauwen P and Schrauwen-Hinderling VB. Carnitine supplementation improves insulin sensitivity and skeletal muscle acetylcarnitine formation in diabetes type II patients (Under review in Diabetologia). 


\section{Publications apart from PhD work (from Previous research experience)}

1. Patel AB*, Veeraiah P, Mohamed S, Kumar MJM, Saba K. Impaired GABAergic and glutamatergic neurometabolic activity in aged mice brain as measured by ${ }^{1} \mathrm{H}-\left[{ }^{13} \mathrm{C}\right]-$ NMR spectroscopy. * Corresponding author, FASEB J. 2021 Feb;35(2): e21321.

2. Saba K, Rajnala N, Veeraiah P, Tiwari V, Rana RK, Lakhotia SC, Patel AB. Energetics of Excitatory and Inhibitory Neurotransmission in Aluminum Chloride Model of Alzheimer's Disease: Reversal of Behavioral and Metabolic Deficits by Rasa Sindoor. Front Mol Neurosci. 2017 Oct 17; 1:323.

3. Tiwari V, Saba K, Veeraiah P, Lakhotia SC, Jose J, Patel AB. Amalaki Rasayana Improved Memory and Neuronal Metabolic Activity in ABPP-PS1 Mouse Model of Alzheimer's Disease. J Biosci. 2017 Sep: 42(3):363-371.

4. Patel AB, Tiwari V, Veeraiah P, Saba K. Increased Astroglial Activity and Reduced Neuronal Function Across Brain in ABPP-PS1 Mouse Model of Alzheimer's Disease. J Cereb Blood Flow Metab. 2017 Jan: 1:271678x17709463.

5. Choi CH, Ha Y, Veeraiah P, Möllenhoff K, Felder J, Shah NJ. Design and Implementation of a Simple Multinuclear MRI System for Ultra High-Field Imaging of Animals. Journal of Magnetic Resonance 2016 Dec: 273:28-32.

6. Singh S, Singh A, Rajkumar R, Katakam SK, Samy SK, Nizamuddin S, Singh A, Shahnawaz S, Peddada V, Khanna V, Veeraiah P, Pandit A, Chaubey G, Singh L, Thangaraj K. Dissecting the Influence of Neolithic Demic Diffusion on Indian Ychromosome Pool through J2-M172 Haplogroup. Nature scientific reports-2016, Jan 12;6:19157.

7. Veeraiah $\mathbf{P}^{\#}$, Noronha $\mathrm{JM}^{\#}$, Maitra S, Khandelwal N, Chakravarty S, Kumar A, Patel AB. Dysfunctional Glutamatergic and $\gamma$-Aminobutyric Acidergic Activities in Prefrontal Cortex of Mice in Social Defeat model of Depression. Biol Psychiatry. 2014 Aug 1;76 (3):231-8. " Equal contribution.

8. Tiwari V, Veeraiah P, Subramaniam V, Patel AB. Differential Effects of Ethanol on Regional Glutamatergic and GABAergic Neurotransmitter Pathways in Mice. J Neurochem. 2014 Mar;128(5):628-40.

9. Gazi N, Tamang R, Singh VK, Ferdous A, Pathak A, Singh M, Anugula S, Veeraiah P, Katakam SK, Yadav B, Reddy A, Rani DS, Qadri SS, Singh L, Chaubey G, Thangaraj K. Genetic Structure of Tibeto-Burman Populations of Bangladesh: Evaluating the Gene Flow along the Sides of Bay-of-Bengal. PloS One. 2013 Oct 9;8(10):e75064. 


\section{Presentations in conferences/ symposiums during PhD work}

\begin{tabular}{|c|c|c|}
\hline Year & Type & Presentation title \& conference details \\
\hline 2021 & Digital poster & $\begin{array}{l}\text { Application of MEGA-sLASER for detection of lipid composition } \\
\text { in the human liver. } 2021 \text { ISMRM Annual meeting \& Exhibition, } \\
\text { 15-20 May, Virtual conference. }\end{array}$ \\
\hline 2020 & Digital poster & $\begin{array}{l}\text { Application of a BIlinear Rotation Decoupling (BIRD) filter for } \\
\text { indirect }{ }^{13} C \text { measurements using J-difference editing in the liver. } \\
28^{\text {th }} \text { Annual meeting of ISMRM 2020, 08-14 Aug, Virtual } \\
\text { conference. }\end{array}$ \\
\hline 2020 & Pitch & $\begin{array}{l}\text { Indirect }{ }^{13} C M R S \text { for the measurement of }{ }^{13} C \text {-enrichment of hepatic } \\
\text { fat in vivo. } 8^{\text {th }} \text { Research day, Dept. of Radiology \& Nuclear } \\
\text { Medicine, } 14^{\text {th }} \text { Feb, Maastricht, The Netherlands. }\end{array}$ \\
\hline 2020 & $\begin{array}{l}\text { Traditional } \\
\text { Poster }\end{array}$ & $\begin{array}{l}\text { Application of a BIlinear Rotation Decoupling (BIRD) filter for } \\
\text { indirect }{ }^{13} C \text { measurements using J-difference editing in the liver. } \\
12^{\text {th }} \text { ISMRM-Benelux Chapter Meeting } 2020,24^{\text {th }} \text { Jan, Arnhem, } \\
\text { The Netherlands. }\end{array}$ \\
\hline 2019 & $\begin{array}{l}\text { Traditional } \\
\text { Poster }\end{array}$ & $\begin{array}{l}T_{2} \text { relaxation times of hepatic water and lipid are associated with } \\
\text { intrahepatic lipid content. NUTRIM Symposium } 2019,27^{\text {th }} \text { Nov, } \\
\text { Maastricht, The Netherlands. }\end{array}$ \\
\hline 2019 & Oral & $\begin{array}{l}\text { Water and lipid } T_{2} \text { are associated with hepatic lipid content. } 36^{\text {th }} \\
\text { Annual Meeting of ESMRMB-2019, 3-5 Oct, Rotterdam, The } \\
\text { Netherlands. }\end{array}$ \\
\hline 2019 & Digital poster & $\begin{array}{l}\text { Water } T_{2} \text { is altered with hepatic lipid fraction. } 27^{\text {th }} \text { Annual Meeting } \\
\text { of ISMRM 2019, 11-16 May, Montreal, Canada. }\end{array}$ \\
\hline 2019 & Digital $\mathrm{p}$ & $\begin{array}{l}\text { High intrahepatic lipid content is associated with low choline status } \\
\text { in humans-a }{ }^{1} \mathrm{H}-\mathrm{MRS} \text { study at } 3 \text { Tesla. } 11-16 \text { May, } 27^{\text {th }} \text { Annual } \\
\text { Meeting of ISMRM } 2019 \text {, Montreal, Canada. }\end{array}$ \\
\hline 2019 & Oral & $\begin{array}{l}\text { Hepatic water and lipid } T_{2} \text { are associated with hepatic lipid content. } \\
9^{\text {th }} \text { Diabetes and Metabolism Research Symposium, } 5^{\text {th }} \text { July, Lille, } \\
\text { France. }\end{array}$ \\
\hline 2019 & Pitch & $\begin{array}{l}\text { State-of- the-art MR spectroscopy towards understanding hepatic } \\
\text { steatosis. } 7^{\text {th }} \text { Research Day-Dept. of Radiology \& Nuclear } \\
\text { Medicine, } 15^{\text {th }} \text { Feb, Maastricht, The Netherlands. }\end{array}$ \\
\hline 2019 & $\begin{array}{l}\text { Traditional } \\
\text { Poster } \\
\text { Nominated for } \\
\text { poster award }\end{array}$ & $\begin{array}{l}T_{2} \text { relaxation times of hepatic water and lipid are associated with } \\
\text { intrahepatic lipid content. } 11^{\text {th }} \text { ISMRM-Benelux Chapter Meeting } \\
2019,17^{\text {th }} \text { Jan, Leiden, The Netherlands. }\end{array}$ \\
\hline
\end{tabular}




\begin{tabular}{|c|c|c|}
\hline Year & Type & Presentation title \& conference details \\
\hline 2018 & $\begin{array}{l}\text { Traditional } \\
\text { Poster }\end{array}$ & $\begin{array}{l}\text { High liver fat content is associated with low choline status in } \\
\text { humans.NUTRIM Symposium 2018, } 21^{\text {st }} \text { Nov, Maastricht, The } \\
\text { Netherlands. }\end{array}$ \\
\hline 2018 & $\begin{array}{l}\text { Traditional } \\
\text { poster }\end{array}$ & $\begin{array}{l}\text { Combination of pre-suppression of lipids and MEGA-sLASER for } \\
\text { the detection of BHB in the human liver. MRS Workshop } 2018 \text { on } \\
\text { Metabolic Imaging, 3-5 Oct, Utrecht, The Netherlands. }\end{array}$ \\
\hline 2018 & $\begin{array}{l}\text { Traditional } \\
\text { Poster }\end{array}$ & $\begin{array}{l}\text { Reproducibility of the measurement of hepatic lipid composition } \\
\text { with }{ }^{1} \mathrm{H} \text { MRS at } 3 T \text {. Joint Annual Meeting ISMRM-ESMRMB } \\
2018,16-21 \text { June, Paris, France. }\end{array}$ \\
\hline 2018 & Pitch & $\begin{array}{l}\text { Lipid composition in hepatic steatosis through }{ }^{1} H-M R S .6^{\text {th }} \\
\text { Research Day-Dept. of Radiology \& Nuclear Medicine, } 14^{\text {th }} \text { Mar, } \\
\text { Maastricht, The Netherlands. }\end{array}$ \\
\hline 2018 & Oral & $\begin{array}{l}\text { Reproducibility of the measurement of hepatic lipid composition } \\
\text { with }{ }^{1} H \text { MRS at } 3 T \text {. ISMRM-Benelux Chapter Meeting } 2018,26^{\text {th }} \\
\text { Jan, Antwerp, Belgium. }\end{array}$ \\
\hline 2017 & Digital poster & $\begin{array}{l}\text { A Novel approach to determine the hepatic lipid composition with } \\
{ }^{1} H \text { MRS at } 3 \text { Tesla. } 34^{\text {th }} \text { Annual Meeting of ESMRMB- 2017, 19- } \\
21 \text { Oct, Barcelona, Spain. }\end{array}$ \\
\hline 2017 & Digital poster & $\begin{array}{l}\text { In vivo multinuclear }{ }^{1} H,{ }^{17} \mathrm{O},{ }^{23} \mathrm{Na} M R I \text { and }{ }^{31} \mathrm{P} M R S \text { of a healthy } \\
\text { rat brain at } 9.4 T .34^{\text {th }} \text { Annual Meeting of ESMRMB- 2017, 19-21 } \\
\text { Oct, Barcelona, Spain. }\end{array}$ \\
\hline 2017 & $\begin{array}{l}\text { Traditional } \\
\text { Poster }\end{array}$ & $\begin{array}{l}\text { A novel approach to determine the hepatic lipid composition with } \\
{ }^{1} H \text { MRS at } 3 \text { Tesla. NUTRIM symposium-2017, 22 }{ }^{\text {nd }} \text { Nov, } \\
\text { Maastricht, The Netherlands. }\end{array}$ \\
\hline 2017 & Pitch & $\begin{array}{l}\text { State-of- the-art MR spectroscopy to assess metabolic } \\
\text { abnormalities in hepatic steatosis. } 5^{\text {th }} \text { Research Day-Dept. of } \\
\text { Radiology \& Nuclear Medicine, } 17^{\text {th }} \text { Feb, Maastricht, The } \\
\text { Netherlands. }\end{array}$ \\
\hline
\end{tabular}

\section{Abbreviations}

ISMRM - International Society for Magnetic Resonance in Medicine (ISMRM)

ESMRMB - European Society for Magnetic Resonance in Medicine and Biology

NUTRIM - School of NUtrition and Translational Research In Metabolism 



\section{ABOUT THE AUTHOR}

Pandichelvam Veeraiah was born on $24^{\text {th }}$ June 1986, in a small village (keelavalavu, nearby Melur) from Tamil Nadu, India, He completed his Bachelor of Technology (B.Tech) in April 2009 at Anna University, India, with a specialization in Biotechnology. During bachelor thesis, he gained research experience in the field of Genomics at the Centre for Cellular and Molecular Biology (CCMB), India. He then continued his research at CCMB until August 2013, at the NMR Microimaging and Spectroscopy laboratory, where he was involved in research projects to study brain energy metabolism using indirect ${ }^{13} \mathrm{C} \mathrm{MR}$ spectroscopy in animal models.

In September 2013, he further continued his scientific career as a guest scientist at the Forschungszentrum (FZJ), Juelich, Germany, where he focused on obesity related metabolic research on rat brain using multinuclear MRS. Subsequently, (in March 2014) he started Master (M.Sc.) in Biomedical Engineering in FH Aachen University of Applied Sciences, Germany. He carried out master thesis on the topic "Optimization and evaluation of multinuclear imaging and ${ }^{31} P$ MR spectroscopy at 9.4 T preclinical scanner" at FZJ, Juelich and received his master degree in June 2016.

In September 2016, he started as a PhD candidate at the Department of Radiology and Nuclear Medicine at the Maastricht University Medical Center (MUMC+), The Netherlands. Pandi worked, under supervision of Dr. Vera Schrauwen-Hinderling, Prof. Wildberger and Dr. Lucas Lindeboom. His PhD work was in close collaboration with the department of Nutrition and Movement Sciences. The research conducted during this period, as described in this $\mathrm{PhD}$ thesis was focused on the development and application of new MRS techniques to investigate the metabolic abnormalities associated with hepatic steatosis in humans. 